\title{
Investigating the Specific Interactions \\ Between Microtubule Stabilising Agents \\ and $\beta$-Tubulin
}

By

Benjamin Jones

A thesis submitted to Victoria University of Wellington in fulfilment of the requirements for the degree of Master in Drug Discovery and Development

Victoria University of Wellington 2017 



\begin{abstract}
Microtubule stabilising agents are a class of cytotoxic compounds that cause mitotic arrest through inhibition of microtubule function. They specifically target $\beta$-tubulin subunits promoting tubulin polymerisation, which eventually leads to cell death. Members of this drug class include the cancer chemotherapeutics paclitaxel and ixabepilone. However, like many cytotoxic agents, tumour cells often develop multi-drug resistance phenotypes limiting the effectiveness of such compounds. This results from the expulsion of these drugs from cells by efflux pumps, as well as mutation of their binding site. Much effort has been focused on improving the utility of this important drug class in the ongoing fight against cancer.
\end{abstract}

The microtubule stabilising agents peloruside $\mathrm{A}$ and laulimalide originate from marine sponge species native to the South Pacific. They have similar pharmacological profiles to paclitaxel and ixabepilone, however with several unique properties. They are poor substrates for efflux pumps and target a different region on $\beta$-tubulin subunits, giving them the potential for treatment of resistant tumours. This represents a novel mechanism of action that may be exploited for drug development, and further characterisation of the binding site is warranted.

The aim of this study is to investigate the contribution of two amino acids of human $\beta I-$ tubulin to the interactions with peloruside A and laulimalide. Specifically, glu127 and lys 124 have been predicted by computational modelling and analogue studies to form hydrogen bonds and other associations with the two compounds. These amino acids are located on $\beta$ tubulin subunits adjacent to the main binding pocket of peloruside $\mathrm{A}$ and laulimalide, and represent a potential inter-protofilament interaction that does not occur with other microtubule stabilising agents. This binding mechanism has not yet been shown by crystallography and is hence based solely on in silico work, requiring biological validation.

HEK293 cells were transfected with $\beta$ I-tubulin with these amino acids mutated to alanines to prevent hydrogen bond formation. Cell proliferation assays, flow cytometry, and immunoblotting were used to study the effect loss of the inter-protofilament interaction has on the bioactivity of peloruside A and laulimalide. These mutations did not significantly alter the concentration-response of cells to either drug in the cell proliferation assay. However, accumulation of cells in the G2/M phase of the cell cycle and the proportion of transfected cells showing signs of mitotic arrest significantly decreased for E127A mutant cells compared to wild type $\beta$ I-tubulin transfected control cells treated with peloruside A. 
Furthermore, a similar reduction in cell cycle block was also seen in E127A mutant cells treated with the negative control ixabepilone, which binds to a different site on $\beta$-tubulin.

No evidence seen in this study suggests that either amino acid plays a major role in peloruside A or laulimalide target binding. However, the amino acid E127 is important for inter-protofilament associations independent of drug treatment, as its mutation appeared to reduce global stability of microtubule structures. This information requires further validation, it may be useful in the design of future analogue syntheses as development of these promising drug candidates continues. 


\section{Acknowledgements}

I would first and foremost like thank my supervisor Prof John H Miller for his continued guidance and support over the past year and allowing me to work with him on this project.

I am also very appreciative for all the work done that has contributed to this project. Principally, Assoc. Prof Paul Teesdale-Spittle and Assoc. Prof Peter Northcote for helping design the mutations and for providing the drugs, respectively.

I am very grateful for the support I have received by everyone in SBS, especially in my first year at Victoria. Special thanks goes to Rosie who helped me out and put up with me so much throughout the year.

I of course must thank my amazing family who I love dearly and miss greatly. If it wasn't for their support I definitely would not be where I am today.

Finally, I owe the world to Georgia for putting up with the late nights and absences over the past few months of madness.

\section{Declaration and Copyright}

The Copyright of this thesis resides with the author. No quotation from it should be published without prior consent and information derived from it should be acknowledged.

The work in this thesis was completed by the author at Victoria university of Wellington, New Zealand. It has not been submitted, in part or in whole, for any higher degree at this or any other University. 


\section{Table of Contents}

Abstract $\quad$ i

Acknowledgements $\quad$ iii

Declaration and Copyright $\quad$ iii

Table of Contents $\quad$ V

List of Abbreviations $\quad$ ix

Chapter 1. Introduction 1

1.1 General Intoduction 1

1.1.1 Natural products 1

1.1.2 Cancer 2

1.1.3 Microtubules 3

$\begin{array}{ll}\text { 1.1.4 Microtubule targeting agents } & 6\end{array}$

$\begin{array}{lll}\text { 1.1.5 Microtubule destabilising agents } & 7\end{array}$

$\begin{array}{lll}\text { 1.1.6 Microtubule stabilising agents } & 8\end{array}$

$\begin{array}{lll}\text { 1.1.7 Peloruside A and laulimalide } & 10\end{array}$

1.1.8 Peloruside A/laulimalide binding site 11

$\begin{array}{lll}1.2 & \text { Research Aims } & 14\end{array}$

1.2.1 Determine the optimum transfection conditions for mutant BI-tubulin in HEK293 cells

1.2.2 Examine the role of the E127 and K124 human BI-tubulin amino acids in the postulated inter-protofilament binding of peloruside $A$ and laulimalide

Chapter 2. Transfection of mammalian cells with mutant $\beta$ I-tubulin to study structure activity relationships

2.1 Introduction 17

2.1.1 Mammalian cell lines as in vitro models of drug target interactions 17

2.1.2 Transfection of exogenous mutant genes 17

$\begin{array}{lll}2.2 & \text { Aims } & 18\end{array}$

2.2.1 Determine optimum conditions to maximise transfection efficiency of wild-type and mutant BI-tubulin plasmids in HEK293 cells 18

2.2.2 Quantify the levels of exogenous BI-tubulin in transfected cells 18

2.2.3 Assess the structure and morphology of microtubules containing exogenous $\beta$ I-tubulin subunits 
$\begin{array}{lll}2.3 & \text { Materials and Methods } & 19\end{array}$

2.3.1 Compounds 19

$\begin{array}{ll}\text { 2.3.2 Cell culture } & 19\end{array}$

2.3.3 Plasmid vector design and preparation 20

2.3.4 Transfection optimisation 22

2.3.5 Transfection for subsequent experiments 23

2.3.6 Quantification of transfection efficiency and cell viability by flow cytometry 23

2.3.7 Quantification of exogenous tubulin expression by western blotting 24

2.3.8 Assessment of microtubule morphology and exogenous tubulin expression by fixed-cell confocal microscopy 25

2.4 Results 26

2.4.1 Quantification and evaluation of wild-type and mutant $\beta$ I-tubulin plasmid $D N A$

2.4.2 Optimisation of transfection conditions

2.4.3 Transfection efficiencies measured by flow cytometry for MTT assays $\quad 30$

2.4.4 Transfection efficiencies measured by western blotting for MTT and cell cycle assays

2.4.5. Mutant $\beta I$-tubulin expression and microtubule morphology examination by immunocytochemistry and confocal microscopy 35

$\begin{array}{lll}2.5 & \text { Discussion } & 38\end{array}$

2.5.1 Expression of exogenous mutant $\beta$ I-tubulin 38

Chapter 3. Assessment of the role of E127 and K124 $\beta$ I-tubulin amino acids in the activity of microtubule stabilising agents 43

3.1 Introduction $\quad 43$

3.1.1 Cytotoxicity screening and MTT assays 43

3.1.2 Cell cycle analysis 43

3.1.3 In situ microtubule polymerisation 44

3.2 Aims 44

3.2.1 Determine the dose-response of peloruside $A$ and laulimalide in cells with BI-tubulin containing either the K124A or E127A point mutations

3.2.2 Evaluate the effect of the E127A mutation on cell cycle arrest caused by peloruside A 
3.2.3 Evaluate the effect of the E127A mutation on in situ microtubule dynamics in response to peloruside $A$

2.3.4 Visualise the effect of the E127A mutation on regular structure of microtubules

3.3 Materials and Methods

3.3.1 Determination of cell activity in E127A and K124A mutants treated with peloruside A and laulimalide by MTT assay

3.3.2 Cell cycle analysis of E127A mutants in response to peloruside A by flow cytometry

3.3.3 In situ polymerisation of E127A mutants in response to peloruside A by western blotting

3.3.4 Assessment of E127A mutant microtubule stabilisation in response to peloruside A by immunocytochemistry and fixed cell confocal microscopy

$3.4 \quad$ Results

3.4.1 Response of transfected HEK293 cell activity to microtubule stabilising agents

3.4.2 Analysis of E127A transfected HEK293 cell cycle arrest caused by peloruside $A$

3.4.3 In situ polymerisation of E127A mutant BI-tubulin in response to peloruside $A$

3.4.4 Examination of microtubule function in response to peloruside A by immunocytochemistry and confocal microscopy

3.5 Discussion

3.5.1 The effect of $\beta I$-tubulin mutations on cell response to microtubule stabilising agents

3.5.2 The changes to cell cycle arrest caused by peloruside A with E127A mutant $\beta I-$ tubulin

3.5.3 In situ polymerisation of exogenous wild-type BI-tubulin treated with peloruside $A$

3.5.4 Qualitative assessment of GFP-tagged microtubule functionality in response to $P L A$ 
4.2 Future Directions

4.3 Conclusion

References 


\section{List of Abbreviations}

1A9 Subclone of the A2780 human ovarian carcinoma cell line

APS Ammonium persulfate

BCA Bicinchoninic acid

bp $\quad$ Base pair

BSA Bovine serum albumin

CMV Cytomegalovirus

D297I Aspartic acid substitution for isoleucine at residue 297

DAPI 4', 6-deamidine-2'-phenylindole

DMEM Dulbecco's modified eagle medium

DMF N, N-dimethylformamide

DMSO Dimethyl sulfoxide

DNA Deoxyribonucleic acid

E127A Glutamate substitution for alanine at residue 127

EDTA Ethylenediaminetetraacetic acid

EGTA Ethylene glycol tetraacetic acid

E-site Exchangeable nucleotide site

FCS Fetal Calf Serum

FDA US Food and Drug Administration

GDP Guanosine 5'-diphosphate

GFP Green fluorescent protein

GTP Guanosine-5'-triphosphate

$\mathrm{HCl} \quad$ Hydrochloric acid

HDX-MS Hydrogen deuterium exchange mass spectrometry

HEK293 Human embryonic kidney cell line

$\mathrm{IC}_{50} \quad$ Half-maximal inhibitory concentration

IXA Ixabepilone

K124A Lysine substitution for alanine at residue 124

LAU Laulimalide

LB Lysogeny broth

M Mitosis phase

MAP Microtubule-associated proteins

MDA Microtubule-destabilising agent 
$\mathrm{MgCl}_{2} \quad$ Magnesium chloride

MSA Microtubule-stabilising agent

MTA Microtubule-targeting agent

MTT 2-(4,5-dimethyl-2thiazolyl)-3,5-diphenyl-2H-tetrazolium bromide

$\mathrm{NaCl} \quad$ Sodium chloride

$\mathrm{NaN}_{3} \quad$ Sodium axide

NIH National Institute of Health

N-site Non-exchangeable nucleotide site

PBS Phosphate buffered saline

PI Propidium iodide

PLA Peloruside A

P-gp P-glycoprotein

PTX Paclitaxel

PVDF Polyvinylidene fluoride

RIPA Radioimmunoprecipitation assay

SDS Sodium dodecyl sulfate

SDS-PAGE Sodium dodecyl sulfate polyacrylamide gel electrophoresis

SEM Standard error of mean

TBS Tris buffered saline

TBST Tris buffered saline with Tween-20

TEMED N,N,N',N'-tetramethylethylenediamine

TRIS Tris(hydroxymethyl)aminomethane

WHO World Health Organisation 


\section{Chapter 1. Introduction}

\subsection{General Introduction}

\subsubsection{Natural products}

Throughout the history of pharmaceutical research and intervention to treat human disease, natural products have been an invaluable supply of lead compounds and chemical scaffolds. ${ }^{1}$ These materials often consist of secondary metabolites that are the result of evolutionary adaptation to a particular environment. They commonly display useful bioactive properties, unique biosynthetic origins, and can be extracted, isolated and altered for therapeutic benefit. For such reasons, drugs from these sources have made up a large proportion of marketed medicines over recent decades. For example, $42 \%$ of all new drugs approved by the United States Food and Drug Administration (US FDA) and similar regulatory agencies during the period 1981 - 2014 are either unaltered natural products, derivatives, thereof or biological macromolecules. ${ }^{2}$ This increases to $67 \%$ if synthetic drugs that either contain a natural product pharmacophore or mimic natural products are included.

A commonly used starting point in many drug discovery efforts is the construction of chemical libraries by the synthesis of a large number of novel organic structures and screening them against one or several biological targets. Alternatively, the use of chemical structures found in nature as the inspiration for medicinal chemistry-driven analogue development has the advantage of beginning with a known and inherent activity in biological systems. Traditionally, this has largely involved compounds of terrestrial plant or microbial origins, however since technological advancements in the mid-twentieth century made underwater exploration more viable the marine environment has proven to be a rich source of these bioactive agents. ${ }^{3}$ Many of these secondary metabolites are produced by microbial species in symbiosis with a host organism as an anti-predation mechanism. ${ }^{4}$ Their protein targets are often essential to cellular function and hence are able to be exploited to modulate disease-related processes in humans. Marine natural products that serve this adaptive purpose are typically very potent due to their dilution upon release into the underwater environment, a valuable property for therapeutic manipulation. ${ }^{5}$

This presents a unique opportunity to utilise chemical structures that are known to already act via specific biological pathways in nature, a useful launch pad for the development of promising lead compounds in drug discovery. These compounds can also be synthetically modified for structure-activity relationship studies (SARs) assisted by modern 
crystallographic, genomic and in silico techniques for specific target identification and reduced off-target effects to produce candidate molecules that have favourable efficacy, specificity and safety profiles. ${ }^{6}$

\subsubsection{Cancer}

Cancer is one of the most prevalent diseases globally and is characterised by overproliferating cells that are extensively mutated, leading to unchecked tumour growth. These mutations often involve genes coding for proliferation, differentiation, DNA repair and apoptosis control. As a collective disease it is responsible for one in four deaths that occur yearly in the USA, ${ }^{7}$ and in New Zealand caused 8,905 deaths from over 21,000 diagnoses in $2012 .^{8}$ It is a particularly relevant health issue in this country with the highest rates of some cancers, such as melanoma, in the world as well as a disproportionately high incidence among Maori and Pacific Island individuals. ${ }^{9}$

The development, growth and progression of cancer involves a number of cellular processes that include the transformation of healthy cells, proliferation and finally migration and invasion. The first stage of development is the acquisition of a malignant phenotype in normal cells, dictated by a number of factors over a long period of time, such as exposure to chemical toxicants. ${ }^{10}$ Cancer development occurs due to genetic mutations that activate oncogenes and inactivate tumour suppressor and stability genes. ${ }^{11}$ These changes cause cells to avoid growth inhibition and pro-apoptosis signals, becoming self-sufficient and able to carry out angiogenesis to supply blood to resulting tumours. ${ }^{12}$ Once cells have transformed, extensive proliferation occurs as a function of their genetic alterations via several different signalling pathways. These include kinase and transcription factor activation leading downstream to increased cell division, as well as genetic alterations that promote the expression of pro-cancer genes. ${ }^{12}$ A further characteristic of cancer cells is their capacity for invasion and metastasis, which is largely dictated by the action of growth factors, phosphatases, metalloproteinases and extracellular matrix proteins. ${ }^{13}$

Despite cancer's high incidence and massive impact on global health, the chances of survival for patients that contract various cancer types have increased consistently over recent decades. ${ }^{7}$ Large-scale efforts worldwide to educate communities about cancer, screening programs allowing for early intervention and concerted efforts in the development of new treatments have likely been the cause of these trends. However, a large proportion of patients 
remain undiagnosed until in late-stage, highly metastatic disease states, and the treatments for many types of cancer provide inadequate prognoses. The most common form of intervention for solid tumours is surgical resection, often combined with other therapies. Limitations to this approach include the inability to remove all disease tissue from affected areas, leading to eventual recurrence of growth, and restrictions depending on the location and type of tumour. ${ }^{14}$ These surgeries are only effective for solid tumours, as opposed to de-localised malignancies such as haematological cancers and leukaemias. Furthermore, tumours can lead to irreparable damage to delicate surrounding tissue, for example in the brain and prostate. ${ }^{15}$ Other common treatment options include radiation therapy, chemotherapy and biological therapy.

Treatment of cancer by traditional chemotherapy typically involves cytotoxic drugs with mechanisms of action that include DNA damage and inhibition of angiogenesis, kinase activity and microtubule function. Particularly relevant to the research proposed here is disruption of microtubule dynamic instability by microtubule targeting agents. However, due to their broad-acting, cytotoxic nature, these drugs often exhibit poor specificity towards tumour cells with common off-target effects that result in severe adverse events. These include damage to non-cancerous, rapidly dividing cells such as hair and gut epithelial cells. ${ }^{16}$ Such limitations have provided major impetus for the development of novel anti-cancer agents with better selectivity towards tumour cells versus normal tissue, as well as new methods for targeted site-specific drug delivery. Another issue often associated with cancer pharmacotherapy is the development of drug resistance through the activity of efflux pumps, the major contributor being P-glycoprotein (P-gp). ${ }^{17}$ In addition, mutations to protein targets can also confer multidrug-resistance to tumour cells. As a result, drug discovery efforts are also being focused on compounds that are not substrates for these pumps and that act via mechanistic targets different to existing treatments. ${ }^{18}$

\subsubsection{Microtubules}

The cytoskeleton of eukaryotic cells is composed of a number of structural proteins and is essential to processes such as cell division, structural maintenance, intracellular transport and motility. Their major role in cell division by meiosis and mitosis involves organelle and chromosomal separation and cytokinesis. ${ }^{19}$ Important components of this cytoskeletal system are microtubules - heterodimeric proteins made up of $\alpha$ - and $\beta$-tubulin subunits. Tubulin 
monomers consist of N-terminal nucleotide-binding, C-terminal helical and central looped domains. $^{20}$ These domains are involved in GTP-binding for polymerisation catalysis, associated protein binding and subunit association, respectively. ${ }^{19}$ The latter process, which results in $\alpha / \beta$ heterodimers, is controlled by several chaperone proteins and cytosolic cofactors. It occurs via polar interactions of tubulin monomers in a head to tail fashion producing extended linear protofilaments. The individual heterodimers within these structures associate laterally, stabilised by the M-loop structural element within the subunits. These inter-dimer associations result in complete microtubule polymers, normally consisting of thirteen protofilaments orientated around a hollow lumen, giving a microtubule diameter of approximately $24 \mathrm{~nm} .^{20,21}$ These polymers are assembled from pre-formed 'microtubule seeds' or structures, the centrioles. ${ }^{22}$ In addition, tubulin exists in various protein species that differ between organisms and tissues, with $\alpha$ - and $\beta$-tubulin being the most common in humans. Within these, isotypes exist that relate to different microtubule-facilitated processes within cells. The isotype composition is both cell and species-specific, depending on the cell structure and functions required. As a result, the transcriptional regulation, post-translational modification and interactions with microtubule-associated proteins (MAPs) of these isotypes are essential to cell survival. ${ }^{23} \beta \mathrm{I}$ is the most ubiquitous of the $\beta$-tubulins among various tissues, and is therefore the focus of this study, as outlined in subsequent sections.

Central to the proper function of microtubules is their characteristic dynamicity, the polymer structure extending or shortening due to the rapid rearrangement of heterodimers in response to cellular requirements (Figure 1.1.1). ${ }^{19}$ This dynamic instability assists in cellular homeostasis and occurs via GTP/GDP catalytic exchange at the N-terminus of $\beta$-tubulin (Esite). This process alters the interactions between adjacent protofilaments by inducing conformational changes within subunits. Bound GTP promotes a straightened tubulin conformation and hence more favourable lateral inter-dimer associations. When rapidly converted to GDP, $\beta$-tubulin shifts to a curved shape causing strain on the polymer, which is partially stabilised by subsequent addition of GTP-bound heterodimers. As a result, when GTP is associated with the tubulin E-site, the equilibrium of microtubule stability is shifted towards polymerisation and therefore promotes overall elongation (rescue). ${ }^{24}$ GTP bound to the exposed $\beta$-tubulin ( + end) acts as a cap, preventing further depolymerisation. Conversely, when this GTP cap is absent or GDP remains in the E-site the curved conformation is less stable and the collapse of inter-dimer interactions is promoted. This may result from GTP hydrolysis outweighing GTP binding or a reduction in available GTP and leads to 
protofilament disassociation and overall microtubule shrinkage (catastrophe) in order to reduce energetic strain. ${ }^{25}$ In contrast to this activity at $\beta$-tubulin subunits, the $\mathrm{N}$-site of $\alpha$ tubulin only binds GTP in a non-exchangeable manner. This promotes greater stability between adjacent subunits when GTP is present, while resulting in less dynamicity at the exposed $\alpha$-tubulin subunits of protofilaments (- end). The opposing mechanisms of rescue and catastrophe together contribute to the diverse regulatory capabilities of microtubules enabling them to carry out a variety of important cellular functions. One such function is chromosome segregation during mitosis wherein microtubule dynamic instability increases during a cell's transition from interphase to the mitotic phase of the cell cycle to assist in their separation through mitotic spindle formation. ${ }^{26}$
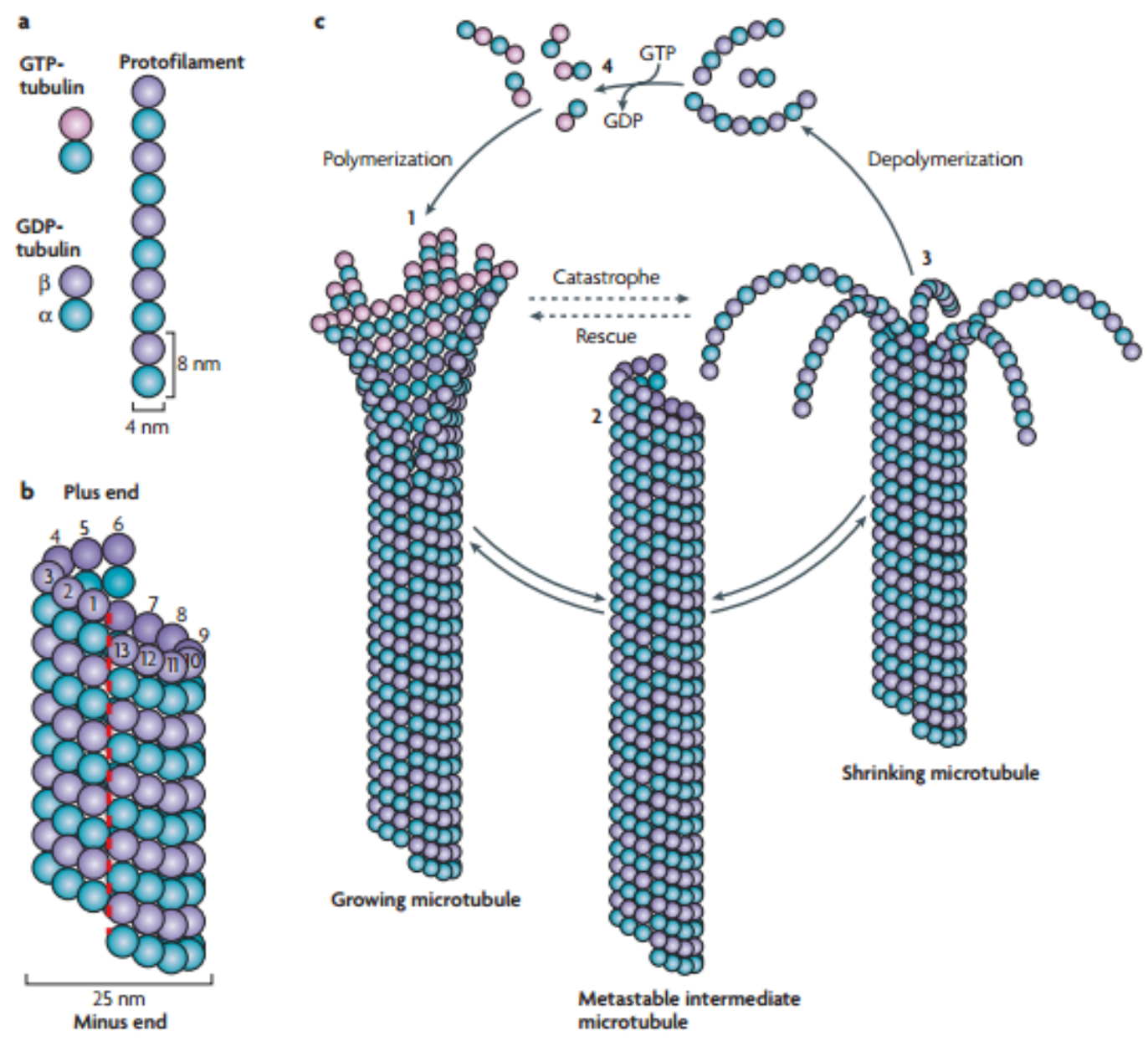

Figure 1.1.1. Microtubule structure and dynamic equilibrium. Schematic showing the structure of microtubules from $\alpha$ - and $\beta$-tubulin heterodimers to protofiliments to cylindrical polymers. Shows the effect of GTP/GDP exchange on dynamicity and the growth and shrinkage of stable and unstable microtubules, respectively. Figure adapted from Akhmanova and Steinmetz (2008) with publisher's permission. 


\subsubsection{Microtubule targeting agents}

Soon after the initial discovery and identification of microtubules in $1963,{ }^{27}$ their subunits were found to be the physiological target of the anti-mitotic compound colchicine. ${ }^{28}$ Since then, colchicine and a number of other agents that act via interactions with these proteins have been classed as microtubule targeting agents (MTAs). These are bioactive compounds, often originating as natural products that disrupt normal microtubule function and the cellular processes they contribute to. Through this mode of action they exert a cytotoxic effect, mostly by cell cycle arrest due to the central role microtubules play in mitosis. This is most commonly observed in cells that are undergoing spindle and aster formation during mitosis. The interference with the microtubule-requiring mitotic process leads to apoptosis via the intrinsic pathway. ${ }^{29}$ As a result, MTAs are most active in rapidly dividing cell types, such as cancer, and hence MTAs have found great use in the treatment thereof. ${ }^{30,31}$ The archetypal MTA is paclitaxel (PTX), which has become a gold standard chemotherapeutic. Drugs that target other mitosis-specific factors have largely failed. ${ }^{32}$ This has been attributed to the inhibition of other non-mitotic process in the cell, such as essential intracellular trafficking, during interphase of the cell cycle as a result of the diversity of dynamic microtubule functions. $^{33}$

Despite, and sometimes due to, the wide and largely successful use of MTAs in the clinic, they are not immune to the off-target side effects and propensity for multidrug resistance that plague other chemotherapeutic drug classes. ${ }^{34}$ As a result, recent efforts have focussed on new methods of targeted MTA drug delivery, such as the use of antibody-drug conjugates. ${ }^{35}$ A number of compounds are also under development that interact with microtubules through novel mechanisms, thus circumventing the resistance of cancers to established treatments. These include some natural product MTAs that are under investigation in this study. MTAs are generally split into two distinct groups: microtubule destabilising agents (MDAs) and microtubule stabilising agents (MSAs). At low concentrations, all MTAs exhibit similar effects in vitro, causing aberrant microtubule function (reduced dynamicity), cell cycle arrest and apoptotic cell death. However, at higher concentrations the two classes exhibit specific and distinct effects dependent on their interaction with the tertiary structure of microtubule polymers. 


\subsubsection{Microtubule destabilising agents}

Upon binding to microtubules, MDAs can inhibit further polymerisation or promote microtubule shortening and eventual total breakdown of the protein into monomers or dimers. ${ }^{36}$ MDAs elicit these effects by binding to one of two unique binding sites on $\beta$ tubulin, specifically the colchicine and vinca binding domains (Figure 1.1.2). ${ }^{31}$ Both of these sites are found on the outer surface of the quaternary microtubule structure.

Colchicine-like compounds target their namesake binding region on $\beta$-tubulin at the interface between adjacent $\alpha$-tubulin subunits. This promotes a curved tubulin conformation and hence polymer instability. ${ }^{37}$ At high concentrations this leads to depolymerisation of the microtubule polymer. Despite displaying potent cytotoxicity, severe off-target effects have hindered clinical approval of these drugs for the treatment of cancer, however they have found use in treating gout by inhibiting neutrophil motility. ${ }^{30}$ Other chemical analogues targeted to this binding site have shown improved activity in recent preclinical studies. ${ }^{38}$

The more successful vinca domain binding agents include vinblastine and vincristine, which are clinically approved frontline chemotherapeutics. ${ }^{39}$ These are thought to cause protein fragmentation due to interference with microtubule regulation and organisation. ${ }^{40}$ They bind $\beta$-tubulin subunits integrated in fully polymerised microtubules at the border of neighbouring heterodimers, and thus impact their essential dynamicity. Despite being useful anti-cancer agents, the efficacy of these drugs is limited by extensive efflux from multidrug resistant tumour cells, spurring the search for novel vinca domain binding agents lacking this property. ${ }^{30}$ 

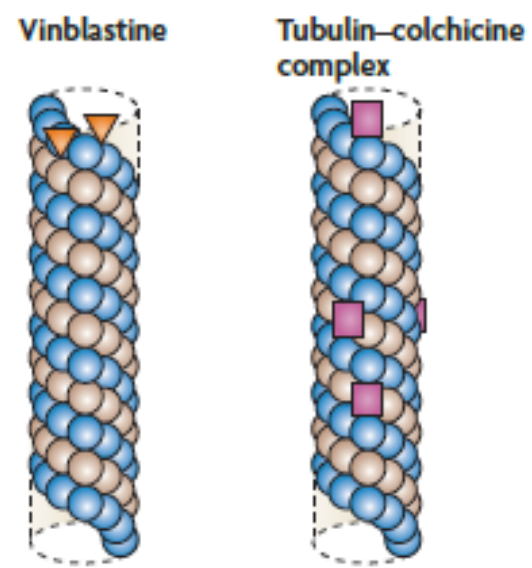

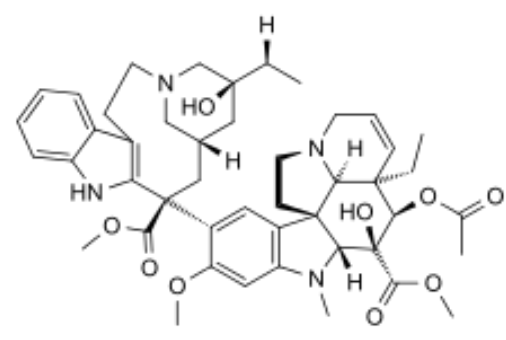

Vinblastine

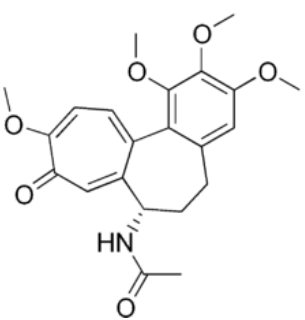

Colchicine

Figure 1.1.2. Microtubule destabilising agent binging regions. Diagram depicting the Vinca domain (orange triangle) and colchicine (pink square) binding regions, situated on the external microtubule wall and targeted by MDAs. Included are the chemical structures for vinblastine and colchicine. Figure adapted from Dumontet and Jordan (2010) with publisher's permission.

\subsubsection{Microtubule stabilising agents}

Upon binding to $\beta$-tubulin, MSAs are able to interfere with microtubule dynamics by stabilising the association of tubulin heterodimers. ${ }^{30}$ Most of these drugs show high affinity for the taxane binding region (Figure 1.1.3), while other more recently discovered marine natural products elicit their cytotoxic effects via a different target site that was first described by David Schriemer's laboratory in Alberta, Canada. ${ }^{41}$ Two such compounds, peloruside A (PLA) and laulimalide (LAU), are the bioactives under investigation in this study.

The taxane compounds include some of the most successful anti-cancer drugs to have been developed in the last few decades, the first and most notable example being the plant natural product paclitaxel (PTX) that was isolated from the Pacific yew tree Taxus brevifolia in 1966. Soon after, its potent cytotoxicity and microtubule-stabilising mode of action were identified. $^{42,43}$ However, it became a quintessential case exemplifying the difficulties often experienced in sustainable large-scale production from scarce natural resources. It was not until 1992 that a semi-synthetic route from a plant culture-derived precursor provided a supply of the drug for clinical use. ${ }^{39}$ PTX binds to the taxane region on GDP-bound $\beta$-tubulin in the microtubule lumen, reducing strain from curvature and leading to the favoured straightened conformation and increased intracellular concentration of the polymerised 
protein. $^{44}$ It has been used extensively to treat a range of cancer types; however, it is associated with limiting pharmacokinetic and dynamic properties such as the requirement for lipophilic drug delivery (Cremophore, EL), which has led to non-compliance clinically due to severe allergic reactions. ${ }^{45}$ It also undergoes extensive efflux from cancer cells as a result of upregulated P-gp activity following long-term use, giving rise to multidrug resistant tumours. ${ }^{46}$ Therefore, current research focusses on ways to avoid these limitation, such as by investigating alternative nanoparticle and antibody delivery systems. ${ }^{47}$

Another class of MSA that binds to the same taxane site and inhibits cell growth at a similar potency to PTX are the epothilones, such as epothilone A and $\mathrm{B}{ }^{48}$ These natural products are of microbial origin, specifically as products of the soil bacterium Sorangium cellulosum, affording the advantage over compounds from plant and marine species in that they can be produced at an industrial scale by fermentation. In addition, their 16-membered structure is considerably simpler than the complex diterpenoid PTX molecule, hence epothilones have a relatively straightforward synthetic route. Clinically, the epothilones have the advantage of increased activity in some multidrug resistant cells as, while it is also a substrate for P-gp, it is not for breast cancer resistance protein efflux pumps. ${ }^{49}$ It also does not require the same lipophilic carrier as PTX due to its higher aqueous solubility. However, both the taxanes and epothilones have been shown to cause off-target adverse effects, namely peripheral neuropathy. ${ }^{50}$ Coupled with poor pharmacokinetics, this drove the search for more clinically useful epothilone analogues. The most advanced of these efforts is the epothilone B derivative, ixabepilone (IXA), which was approved by the FDA in 2007 for refractory metastatic breast cancer. ${ }^{51}$

Taccalonolides are yet another group of plant-derived compounds that promote microtubule polymerisation; however, the precise mechanism by which this occurs is yet to be fully elucidated. It has recently been shown that while retaining cytotoxicity in cells with taxane binding site mutations, taccalonolide analogues appear to interact covalently with the taxane site region. ${ }^{52,53}$ Most taccalonolides have a growth inhibitory $\mathrm{IC}_{50}$ in the $\mu \mathrm{M}$ range, but some are active in the low nM range. Due to their unique microtubule association and lack of efflux through P-gp proteins, further research into the mechanism of action of these compounds may lead to future improvements to MSA cancer therapeutics.

The majority of MSAs come from marine sources, most of which also act via the same taxane site. Some such agents, for example zampanolide, cause potent polymerisation in the low $\mathrm{nM}$ 
range. ${ }^{54}$ Four MSAs, the taccalonolides, zampanolide, dactylolide and cyclostreptin covalently bind to $\beta$-tubulin, generating significant interest the importance of that binding to their mechanism of action. ${ }^{55,56}$ However, as with many of the non-microbial natural product MSAs discovered, development has been hindered by difficulties in isolation and sustainable synthesis. Distinct from all of these bioactive compounds, LAU and PLA are two marine natural products that exhibit a different mechanism of microtubule stabilisation. Specifically, they bind to a non-taxane site on $\beta$-tubulin affording them unique properties compared to their predecessors. ${ }^{46}$ It is this interaction between LAU/PLA and $\beta$-tubulin that will be addressed by the research presented here.

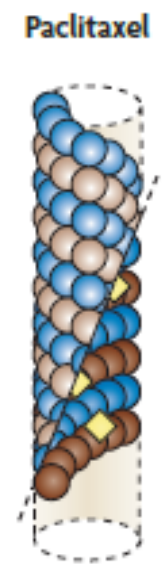

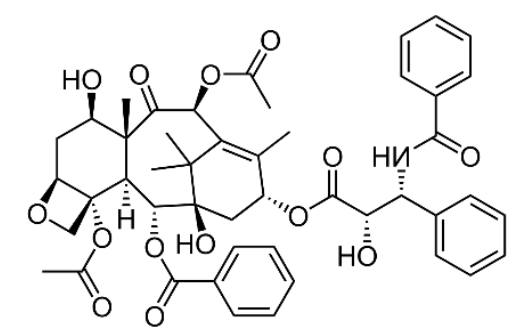

Paclitaxel

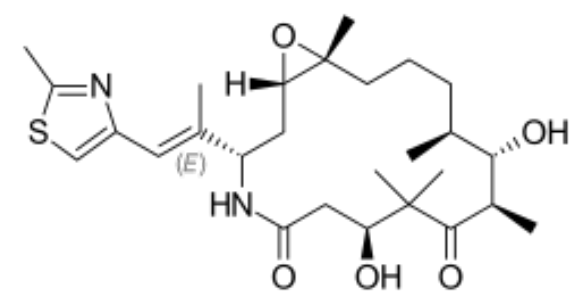

Ixabepilone

Figure 1.1.3. Taxane binding region. Diagram depicting the taxane (yellow diamond) binding region, found in the luminal space and the target of most MSAs. Included are the chemical structures for PTX and IXA. Figure adapted from Dumontet and Jordan (2010) with publisher's permission.

\subsubsection{Peloruside A and laulimalide}

PLA is a polyketide compound sourced from the marine sponge Mycale hentscheli native to the Pelorus Sound in the South Island of New Zealand. ${ }^{57}$ This 16-membered macrolide exhibits potent in vitro anti-cancer activity via microtubule stabilisation similar to traditional MSAs such as PTX. ${ }^{58}$ More recently, this effect has been shown to carry over into mouse xenograft models of lung and breast cancer, outperforming commonly used chemotherapeutics in reducing tumour size. ${ }^{59}$ As demonstrated via competition studies, it binds $\beta$-tubulin at the same target site as LAU and was also found it to be a poor substrate for P-gp drug efflux proteins. ${ }^{46}$ Together, these properties suggest that PLA may be a useful anticancer agent where taxane site mutations or multidrug resistance has rendered other drugs 
ineffective. Studies have also displayed the potential for exploiting the orthogonality of binding sites for LAU/PLA and other MSAs through synergistic combination chemotherapy. ${ }^{60}$

LAU was first isolated from the marine sponges Hyattella sp. and Cacospongia mycofijiensis in $1988 .^{61,62}$ It is a 20-membered polyketide and potent MSA exhibiting nanomolar cytotoxicity in vitro. ${ }^{63}$ It was found not to bind to the taxane site as it did not compete for tubulin binding with PTX, nor did it lose activity in cells resistant to taxane and epothilone compounds. ${ }^{64}$ It was shown to be a poor substrate for P-gp, retaining effectiveness in cancer cells resistant to PTX and epothilone A and B due to taxane binding site mutations, as well as those overexpressing the efflux pumps. In vivo, LAU's initial promise has translated well to some animal cancer models, ${ }^{65}$ however inconsistent inhibitory effects were seen for others. ${ }^{66}$ It has been suggested that this is due to a lack of bioavailability owing to its high lipophilicity. ${ }^{63}$

\subsubsection{Peloruside A/laulimalide binding site}

The use of LAU or PLA as a chemical scaffold for the generation of synthetic analogues may result in the development of a clinically useful, next-generation MSA anti-cancer drug. To that end, several studies have endeavoured to fully elucidate their precise mode of binding to tubulin and determine which specific protein amino acids are involved. Initial computer docking by Pineda et al. (2004) suggested they act on $\alpha$-tubulin; ${ }^{67}$ however, it has since been confirmed that an exterior site on $\beta$-tubulin is in fact the target.

X-ray crystallography has been used in the past to investigate the interactions between MTAs, such as PTX, and microtubules, however this was not possible in the early studies of LAU and PLA. ${ }^{68}$ This is due to a lack of $2 \mathrm{D}$ crystalline tubulin sheet formation on $\mathrm{Zn}^{2+}$ supports used to supply diffraction data in the case of PTX. ${ }^{69}$ Therefore, other methods, including hydrogen-deuterium exchange mass spectrometry (HDX-MS) and $\mathrm{H}^{3}$-radiolabelled binding assays, have been used to discover the exact location of the PLA/LAU binding region. These studies provided information on the orientation of the molecules within a proposed binding pocket that would give favourable hydrophobic interactions and hydrogen bonding. ${ }^{41,70}$ 
The binding of these compounds near the border between $\beta$-tubulin subunits the adjacent protofilaments was eventually concluded, despite initial work leading to contradicting models. This was suggested to cause strengthened inter-protofilament associations through M-loop stabilisation. ${ }^{71}$ The amino acids involved in these interactions were further characterised by Prota et al. (2014) using X-ray crystallography of PLA and LAU bound to tubulin. $^{72}$ This group succeeded in producing a crystal structure of tubulin complexed with PLA, LAU and epothilone A. $\alpha / \beta$-tubulin complex was created that allowed for $\mathrm{x}$-ray diffraction without the need for $\mathrm{Zn}^{2+}$ tubulin crystal structures. The results supported the previous work and gave important information as to which oxygen atoms form hydrogen bonds with which amino acids (Figures 4.1.4 and 4.1.5). In order to improve the efficacy and specificity of these exciting lead compounds the predicted interactions require verification in terms of their role in the bioactivity of PLA and LAU. This was done using in vitro studies of drug-resistant cell lines and site-directed mutagenesis to experimentally isolate and evaluate certain amino acids. Specifically, the generation of PLA-resistant cancer cell lines gave evidence that R308, A298, Y340 and N337 were crucial to tubulin binding, supporting previous computational docking models. ${ }^{73}$ Likewise, mutagenic studies using $\beta$ I-tubulin with four selected point mutations showed that the conversion of D297 to an isoleucine conferred resistance to HEK293 cells, again suggesting an important role for this particular residue. ${ }^{74}$

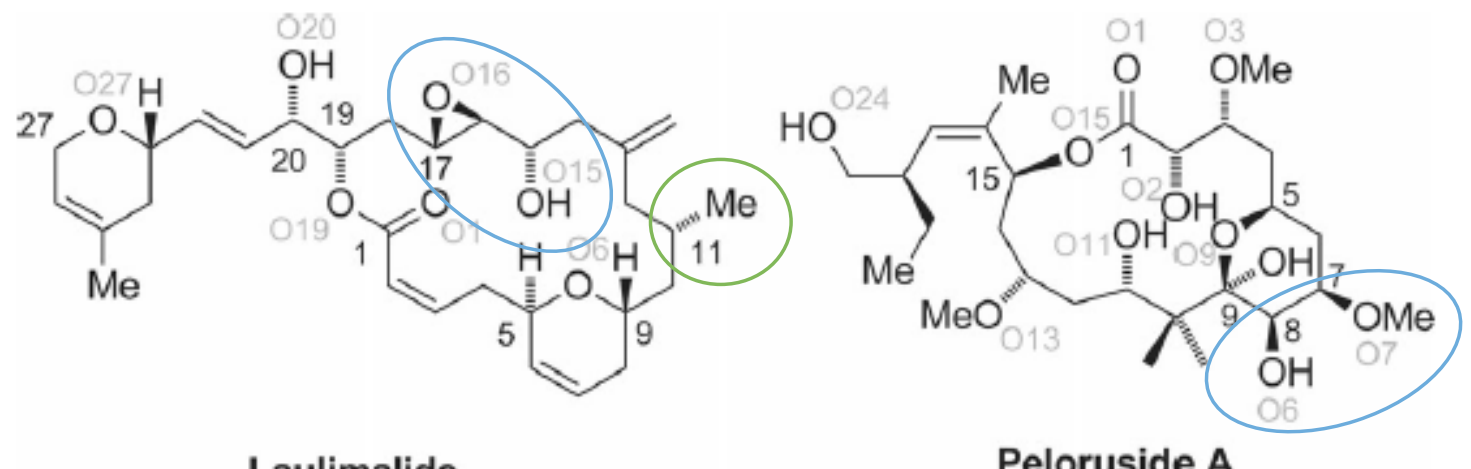

\section{Laulimalide}

Peloruside A

Figure 1.1.4. Laulimalide and peloruside A. Chemical structures of LAU and PLA. Highlighted in blue are the oxygen atoms that have been modelled to form interprotofilament hydrogen bonds with specific amino acids on adjacent $\beta$-tubulin subunits. The green circle indicates a methyl group on LAU this is predicted to be involved in hydrophobic interaction with the E127 residue under investigation here. Figure adapted from Prota et al. (2014) with publisher's permission. 
Of particular interest to this thesis, computer modelling has predicted that several amino acids facilitate a potential interaction with adjacent protofilaments within the microtubule polymer. One particular amino acid, E127, is within close proximity to a methyl group at the 11position on LAU when in its binding pocket and may come within hydrogen bonding range of oxygen-containing groups on PLA and LAU with associated conformational changes in $\beta$ tubulin. Another amino acid, K124, is predicted to form hydrogen bonds with oxygens at the 15 and 16-positions on LAU and 6 and 7-positions on PLA. ${ }^{72,75}$ The importance of hydrogen bonds formed by these particular atoms on PLA is supported by analogue studies that show a $>1000$-fold loss in activity when these hydrogen bond donors are modified. ${ }^{76}$ This inter-dimer bridging may explain the synergy observed between PLA/LAU and other taxane-site MSAs, and hence confirmation of these interactions will provide valuable information about the compounds' mechanism of action. 


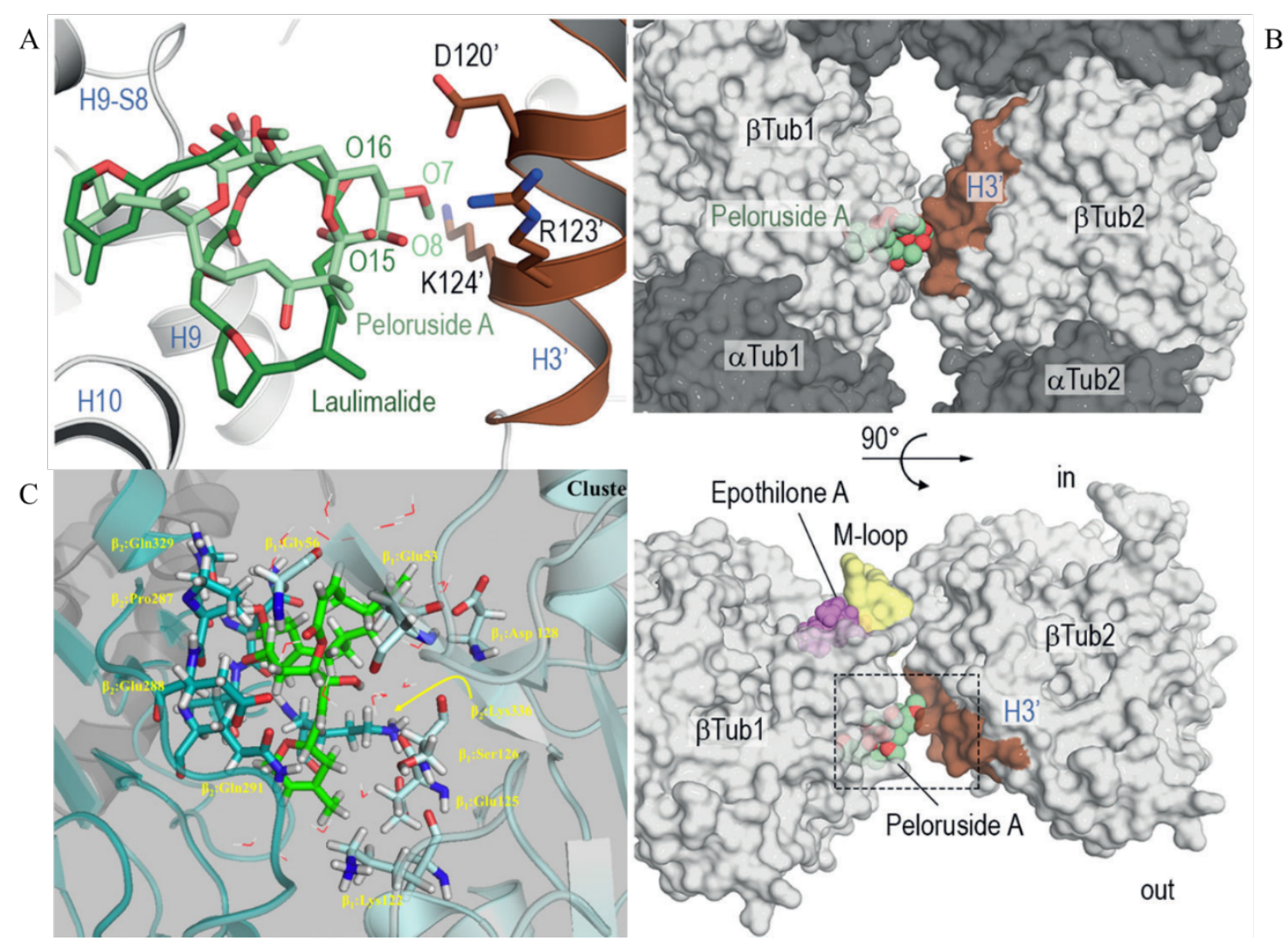

Figure 1.1.5. Peloruside A/laulimalide binding region. (A) and (B) show the PLA/LAU binding site as determined by X-ray crystallography. The former shows the proximity of each molecule to key amino acids on the adjacent $\beta$-tubulin monomer (brown) as determined by computer modelling. The latter demonstrates the location of this binding pocket in relation to the inter-protfilament border and disparate taxane site (pink). (C) shows the results of a different modelling experiment and illustrates the potential role of the E127 residue (Glu125) in LAU binding. Figure adapted from Prota et al. (2014) and Churchill et al. (2015) with publishers' permission.

\subsection{Experimental Design}

\subsubsection{Determine the optimum transfection conditions for mutant BI-tubulin in HEK293 cells}

The effect of the E127A and K124A mutations on the sensitivity of cells to LAU or PLA will depend on the level of expression of mutant $\beta I$-tubulin throughout the entire cell population. In order to draw appropriate inferences from the results, it is important to establish the efficiency of the transfection procedure to allow maximisation of the expression of mutant protein. This will be done using fluorescence microscopy and flow cytometry to determine the proportion of successfully transfected cells. In addition, Western blotting will be used to 
determine the intracellular concentration of mutant compared to endogenous $\beta I$-tubulin. It is also important when interpreting the observed effects of amino acid substitutions that the mutant protein is biologically functional and does not contribute to changes in cell viability in the absence otest compounds. Therefore, confocal microscopy will be used to examine the successful incorporation of mutant $\beta \mathrm{I}$-tubulin into microtubule structures within transfected cells. This will be facilitated by the GFP tag on transfected tubulin.

\subsubsection{Examine the role of the E127 and K124 human BI-tubulin amino acids in the} postulated inter-protofilament binding of peloruside A and laulimalide

This study will investigate two specific amino acids that lie at the inter-protofilament border between adjacent $\beta$ I-tubulin subunits within the overall microtubule structure. These have been proposed to play an important role in PLA/LAU binding and their subsequent microtubule stabilising action. The importance of this inter-protofilament interaction will therefore be studied by transfecting cultured human embryonic kidney (HEK293) cells to express $\beta$ I-tubulin with the mutation E127A or K124A. The mutant tubulin will be a fusion protein with a green fluorescent protein (GFP) reporter. The effects of these substitutions on the ability of PLA or LAU to inhibit proliferation by arresting cells in mitosis will be tested using a cell proliferation assay to determine $\mathrm{IC}_{50}$ concentrations, flow cytometry testing for inhibition of cell cycle progression, and intracellular tubulin polymerisation assays to directly analyse PLA/LAU-tubulin chemical interactions. Mock-transfected cells and cells transfected with wild-type $\beta$ I-tubulin will be used as controls. $\beta$ I-tubulin containing a previously studied mutation (D297I) that has known effects on the action of LAU and PLA will also be used as a positive control. These will allow any changes in the concentration-response to be attributed to the specific amino acids under investigation. In addition, the taxane MSAs, PTX and IXA, will be used to determine if the mutations affect tubulin protein in a global fashion due to the known orthogonality between the binding sites of different MSA classes. This conformational impact can also be tested by examining the stabilising activity of the mutant tubulin cells in the absence of an MSA. It is therefore hypothesized that the E127A and K124A mutations may confer resistance specifically to LAU or PLA. This research will verify the previously computer-modelled binding predictions and provide direct biological evidence for the importance of inter-protofilament interactions in microtubule stabilisation. 


\section{Chapter 2. Transfection of mammalian cells with mutant $\beta I$-tubulin to study structure activity relationships}

\subsection{Introduction}

\subsubsection{Mammalian cell lines as in vitro models of drug target interactions}

The use of cell-based biological assays is an important step in the drug development pipeline and often follows on from initial chemical discovery, while preceding preclinical tests in animal disease models. Although there are various ways in which these experiments may be carried out, as well as purposes they may serve, a common model is the mammalian cell line. While not representative of the entire biological systems that can contribute to a drug's activity in treating a disease, cell lines are useful to rapidly screen compound libraries against specific intracellular targets, or to more closely probe the interactions between lead compounds and those protein targets. This is the case for the present study, which seeks insight to the specific molecular binding mode of PLA and LAU to $\beta$-tubulin and hence the wider disease context is not needed.

Other forms of in vitro study may include the use of purified protein or less complex model organisms such as yeast. Due to the aberrant microtubule polymer structure that forms in isolation from mammalian cells, the former is not appropriate in the present investigation. While yeast studies are useful, particularly when utilising gene editing techniques, their greater insensitivity to MSAs reduces their utility in teasing out any small differences in cytotoxicity that may be present. Furthermore, the greater economy of mammalian systems is important in natural products studies where drug supply is limited. Another advantage of using mammalian cells is the large diversity of cell types available for use, which can be selected based on the specific requirements of an investigation. For example, in this case, HEK293 cells have been chosen as they are rapidly dividing, therefore exhibiting greater microtubule activity than other cell lines. In addition, they are relatively easy to transfect with plasmid DNA and have hence become the archetypal cell line for use in transfection studies.

\subsubsection{Transfection of exogenous mutant genes}

To study the effects of the mutations E127A and K124A on the activity of PLA and LAU, mutant $\beta$ I-tubulin subunits have been introduced into HEK293 cells. To this end, cationic liposome transfection reagents were used to transport plasmid DNA sequences into the 
cytoplasm, and further to the nucleus, of cells in order for it be transcribed and the exogenous genes expressed. The plasmid vector employed contained the sequence encoding $\beta I$-tubulin and the desired point mutation, followed downstream by a sequence coding for green fluorescence protein (GFP) to be used as a marker for successful transfection, to enable the assessment of transfection efficiency and microtubule morphology. The transfection reagents used involve cationic lipid molecules that complex with negatively charged DNA. ${ }^{77}$ This enables fusion with the negatively charged cell membrane, carriage of nucleic acids into the cytoplasm by endocytosis and subsequent assistance of safe passage into the nucleus.

\subsection{Aims}

2.2.1 Determine optimum conditions to maximise transfection efficiency of wild-type and mutant $\beta I$-tubulin plasmids in HEK293 cells

In order to successfully discover the full role that the E127 and K124 amino acids play in PLA and LAU binding, a high proportion of cells will require the associated point mutation. Therefore, to achieve the highest power of inference, the transfection protocol will be optimised. This will involve maximising transfection efficiency and consistency of both wildtype and mutant exogenous $\beta$ I-tubulin in HEK293 cell populations.

\subsubsection{Quantify the levels of exogenous BI-tubulin in transfected cells}

Any changes observed in the response of cells to PLA and LAU upon transfection with either of the $\beta I$-tubulin mutations will likely be proportional to both the amount of exogenous tubulin present within individual cells as well as the percentage cells within a population expressing the mutations. Therefore, it is important to determine the abundance of mutant tubulin in each concentration-response experiment in order to properly inform any conclusions drawn.

\subsubsection{Assess the structure and morphology of microtubules containing exogenous $\beta I-t u b u l i n$} subunits

The successful integration of exogenous tubulin within a cell's microtubule polymers will affect any impact point mutations may have on drug response. It is therefore crucial to assess 
whether this is occurring in the experiments performed here. This will be carried out using fixed cell confocal microscopy to qualitatively observe microtubule morphology within cell samples.

\subsection{Materials and Methods}

\subsubsection{Compounds}

PLA and LAU were provided by Associate Professor Peter Northcote (Victoria University of Wellington, New Zealand). These compounds were isolated and purified from the marine sponge species Cacospongia mycofijiensis and Mycale hentsheli, respectively. $C$. mycofijiensis was sourced from 'Eua and Vava'u in Tonga, while M. hentsheli was collected from the Pelorus Sound in the South Island of New Zealand. Following purification, both drugs were solubilised in DMSO and stored at $-80^{\circ} \mathrm{C}$. PTX (Taxol $\left.{ }^{\circledR}\right)$ was purchased form Sigma Chemical Co. (St. Louis, MO, USA), while IXA (Ixempra $\left.{ }^{\circledR}\right)$ was purchased from Bristol-Meyers Squibb (New York City, NY, USA). These compounds were also dissolved in DMSO and stored at $-80^{\circ} \mathrm{C}$. All of the above drugs were kept as $10 \mathrm{mM}$ stock solutions, which were diluted to $100 \mu \mathrm{M}$ working solutions in DMEM medium (Invitrogen) at the time of cell treatment.

\subsubsection{Cell culture}

HEK293 cells (ATCC, In Vitro Technolgies, New Zealand) were used for all of the experiments in this investigation and were confirmed to be free of mycoplasma contamination. Handling of cell cultures was carried out in a sterile environment within a Logic Labconco ${ }^{\circledR}$ Purifier ${ }^{\circledR}$ Biological Safety Cabinet (Total Lab Systems Ltd., New Zealand). Cells were cultured in DMEM medium (Invitrogen) supplemented with $10 \%$ fetal calf serum (FCS) (Invitrogen), 100 units/mL penicillin/streptomycin antibiotic (Invitrogen) and $2 \mu \mathrm{M}$ Glutamax ${ }^{\mathrm{TM}}$ (Life Technologies), unless otherwise specified. Cells were grown in 25 and $75 \mathrm{~cm}^{2}$ culture flasks (Falcon ${ }^{\circledR}$, In Vitro Technologies) in a humidified incubator (SANYO Electric Co. Ltd.) at $37^{\circ} \mathrm{C}$ with $5 \% \mathrm{CO}_{2}$ in air. Cells were harvested or passaged when they reached $70 \%$ to $90 \%$ confluence. Medium was aspirated and cells detached by first washing with 1 x PBS and adding $600 \mu \mathrm{L}$ TrypLETM Express (Life Technologies) and incubating at $37^{\circ} \mathrm{C}$ for $4 \mathrm{~min}$ (quantities for $25 \mathrm{~cm}^{2}$ flasks). Cells were suspended in $5 \mathrm{~mL}$ 
DMEM and centrifuged for $4 \mathrm{~min}$ at $300 \mathrm{~g}$ and aspirated to remove the medium containing TrypLETM. The remaining cell pellet was resuspended in $5 \mathrm{~mL}$ DMEM before seeding $2 \mathrm{~mL}$ into $5 \mathrm{~mL}$ fresh DMEM in new culture vessels. Cell stocks were stored in liquid nitrogen in Cryo. s cryotubes (Greiner Bio-one) at $1 \times 10^{6}$ cells $/ \mathrm{mL}$ in freeze-down medium (FCS with $16 \%$ DMSO (v/v)). Cells were cultured for a maximum of 15 passages after thawing from liquid nitrogen to ensure a consistent genotype across all experiments.

\subsubsection{Plasmid vector design and preparation}

The PrecisionShuttle mammalian vector with C-terminal TurboGFP flag pCMV6-AC-GFP (Origene Technologies, Rockville, MD, USA) was used for the expression of human tGFP$\beta I-t u b u l i n$ in HEK293 cells driven by a cytomegalovirus (CMV) promoter (Figure 2.3.1). A single point mutation of either amino acid 124 (lysine) to alanine or 127 (glutamate) to alanine of the human $\beta \mathrm{I}$-tubulin gene sequence in plasmid RG203629 (Origene) was incorporated via cloning done by Mutagenex (NJ, USA) and the entire construct transformed into E. coli. To generate plasmid stocks for experimentation, lysogeny broth (LB) agar (1\% Bacto-tryptone, 5\% Bacto-yeast extract, $1 \% \mathrm{NaCl}, 1 \%$ agar) with $100 \mu \mathrm{g} / \mathrm{mL}$ ampicillin (Sigma-Aldrich, Auckland, New Zealand) was inoculated with E. coli bacteria containing either mutant $\beta$ I-tubulin tGFP plasmid or wild-type tGFP- $\beta$ I-tubulin plasmid. The bacteria were cultured for $16 \mathrm{~h}$ at $37^{\circ} \mathrm{C}$, then spread at limiting dilution onto an agar plate, after which time a single positive colony was selected for each plasmid based on ampicillin resistance. These were inoculated in $200 \mathrm{~mL}$ sterile LB broth with $100 \mu \mathrm{g} / \mathrm{mL}$ ampicillin and incubated for $16 \mathrm{~h}$ at $37^{\circ} \mathrm{C}$ while shaking at $220 \mathrm{rpm}$ (Bioline Shaking Incubator, Edwards Instrument Co., Australia). The cultures were then centrifuged at $5000 \mathrm{~g}$ for $10 \mathrm{~min}$ in a SORVALL RC 6+ Ultrafuge (ThermoScientific). Subsequent plasmid purification was carried out using a Hi Speed Plasmid Maxi kit (Qiagen, Australia) according to the manufacturer's instructions. The resulting plasmid concentration was quantified using an ND-1000 Nanodrop spectrophotometer (Thermofisher Scientific). The quality of the samples was determined by electrophoresis of $2 \mu \mathrm{L}$ of plasmid solution in a 1:100 dilution in a $1 \%$ agarose gel for $1 \mathrm{~h}$ at $100 \mathrm{~V}$ alongside a $1 \mathrm{~kb}$ DNA ladder (Life Technologies). 


\section{Lys124Ala:}

GGA.TTCGTCGACTGGATCCGGTACCGAGGAGATCTGCCGC\&GCGATCGCDATGAGGGAAATCGTGCACATCCAGGCTGGTCA GTGTGGCAACCAGATCGGTGCCA.AGTTCTGGGAGGTGATCAGTGATGA.ACATGGCATCGACCCCACCGGCACCTACCACGGGG ACAGCGACCTGCAGCTGGACCGCATCTCTGTGTACTACAATGAAGCCACAGGTGGCAAATATGTTCCTCGTGCCATCCTGGTG GATCTAGAACTGGGACCATGGACTCTGTTCGCTCAGGTCCTTTTGGCCAGATCTTTAGACCAGACAACTTTGTATTTGGTCA GTCTGGGGCAGGTAACA.ACTGGGCCAAAGGCCACTACACAGAGGGCGCCGAGCTGGTTGATTCTGTCCTGGATGTGGTACGGg CGGAGGCAGAGAGCTGTGACTGCCTGCAGGGCTTCCAGCTGACCCACTCACTGGGCGGGGGCACAGGCTCTGGAATGGGCACT CTCCTTATCAGCAAGATCCGAGA.AGA.AACCCTGATCGCATCATGAATACCTTCAGTGTGGTGCCTTCACCCA_AGTGTCTGA CACCGTGGTCGAGCCCTACAATGCCACCCTCTCCGTCCATCAGTTGGTAGAGA.ATACTGATGAGACCTATTGCATTGACA.ACG AGGCCCTCTATGATATCTGCTTCCGCACTCTGAAGCTGACCACACCAACCTACGGGGATCTGA_ACCACCTTGTCTCAGCCACC ATGAGTGGTGTCACCACCTGCCTCCGTTTCCCTGGCCAGCTCA.ATGCTGACCTCCGCAAGTTGGCAGTCA.ACATGGTCCCCTT CCCACGTCTCCATTTCTTTATGCCTGGCTTTGCCCCTCTCACCAGCCGTGGA_GCCAGCAGTATCGAGCTCTCACAGTGCCGG A.ACTCACCCAGCAGGTCTTCGATGCCAAGA_AATGATGGCTGCCTGTGACCCCCGCCACGGCCGATACCTCACCGTGGCTGCT GTCTTCCGTGGTCGGATGTCCATGA_AGAGGTCGATGAGCAGATGCTTA_AGTGCAGA_CA.AAACAGCAGCTACTTTGTGGA ATGGATCCCCAACA.ATGTAAGACAGCCGTCTGTGACATCCCACCTCGTGGCCTCAAGATGGCAGTCACCTTCATTGGCAATA GCACAGCCATCCAGGAGCTCTTCAAGCGCATCTCGGAGCAGTTCACTGCCATGTTCCGCCGGA.AGGCTTCCTCCACTGGTAC ACAGGCGAGGGCATGGACGAGATGGAGTTCACCGAGGCTGAGAGCA.ACATGA.ACGACCTCGTCTCTGAGTATCAGCAGTACCA GGATGCCACCGCAGAAGAGGAGGAGGATTTCGTGAGGAGGCCGA_GAGGAGGCDACGCGTACGCGGCCGCTCGAGATGAGA GCGACGAGAGCGGCCTGCCCGCCATGGAGATCGAGTGCCGCATCACCGGCACCCTGAACGGCGTGGAGTTCGAGCTGGTGG

\section{Glu127Ala:}

GGA.TTCGTCGACTGGATCCGGTACCGAGGAGATCTGCCGCOGCGATCGCCATGAGGGA_ATCGTGCACATCCAGGCTGGTCA GTGTGGCA.ACCAGATCGGTGCCA.AGTTCTGGGAGGTGATCAGTGATGAACATGGCATCGACCCCACCGGCACCTACCACGGGG ACAGCGACCTGCAGCTGGACCGCATCTCTGTGTACTACA_ATGAAGCCACAGGTGGCA.ATATGTTCCTCGTGCCATCCTGGTG GATCTAGA.ACCTGGGACATGGACTCTGTTCGCTCAGGTCCTTTTGGCCAGATCTTTAGACCAGACA.ATTTGTATTTGGTCA GTCTGGGGCAGGTAACA.ACTGGGCCA.AGGCCACTACACAGAGGGCGCCGAGCTGGTTGATTCTGTCCTGGATGTGGTACGGA AGGAGGCAGCGAGCTGTGACTGCCTGCAGGGCTTCCAGCTGACCCACTCACTGGGCGGGGGCACAGGCTCTGGA.AGGGCACT CTCCTTATCAGCA_AGATCCGAGA.AGA.ATACCTGATCGCATCATGA_ATACCTTCAGTGTGGTGCCTTCACCCA_AGTGTCTGA CACCGTGGTCGAGCCCTACAATGCCACCCTCTCCGTCCATCAGTTGGTAGAGA.ATACTGATGAGACCTATTGCATTGACA.ACG AGGCCCTCTATGATATCTGCTTCCGCACTCTGAAGCTGACCACACCAACCTACGGGGATCTGAACCACCTTGTCTCAGCCACC ATGAGTGGTGTCACCACCTGCCTCCGTTTCCCTGGCCAGCTCA_TGCTGACCTCCGCAAGTTGGCAGTCA.ACATGGTCCCCTT CCCACGTCTCCATTTCTTTATGCCTGGCTTTGCCCCTCTCACCAGCCGTGGAAGCCAGCAGTATCGAGCTCTCACAGTGCCGG A.ACTCACCCAGCAGGTCTTCGATGCCAAGA.ACATGATGGCTGCCTGTGACCCCCGCCACGGCCGATACCTCACCGTGGCTGCT GTCTTCCGTGGTCGGATGTCCATGA.AGGAGGTCGATGAGCAGATGCTTA_ACGTGCAGA.ACA.GA_ACAGCAGCTACTTTGTGGA ATGGATCCCCA_A CA.ATGTCAAGACAGCCGTCTGTGACATCCCACCTCGTGGCCTCAAGATGGCAGTCACCTTCATTGGCA.ATA GCACAGCCATCCAGGAGCTCTTCAAGCGCATCTCGGAGCAGTTCACTGCCATGTTCCGCCGGAAGGCCTTCCTCCACTGGTAC ACAGGCGAGGGCATGGACGAGATGGAGTTCACCGAGGCTGAGAGCAACATGAACGACCTCGTCTCTGAGTATCAGCAGTACCA GGATGCCACCGCAGA.AAGGAGGAGGATTTCGGTGAGGAGGCCGAAGAGGAGGCCACGCGTACGCGGCCGCTCGAGATGGAGA GCGACGAGAGCGGCCTGCCCGCCATGGAGATCGAGTGCCGCATCACCGGCACCCTGAACGGCGTGGAGTTCGAGCTGGTGG 


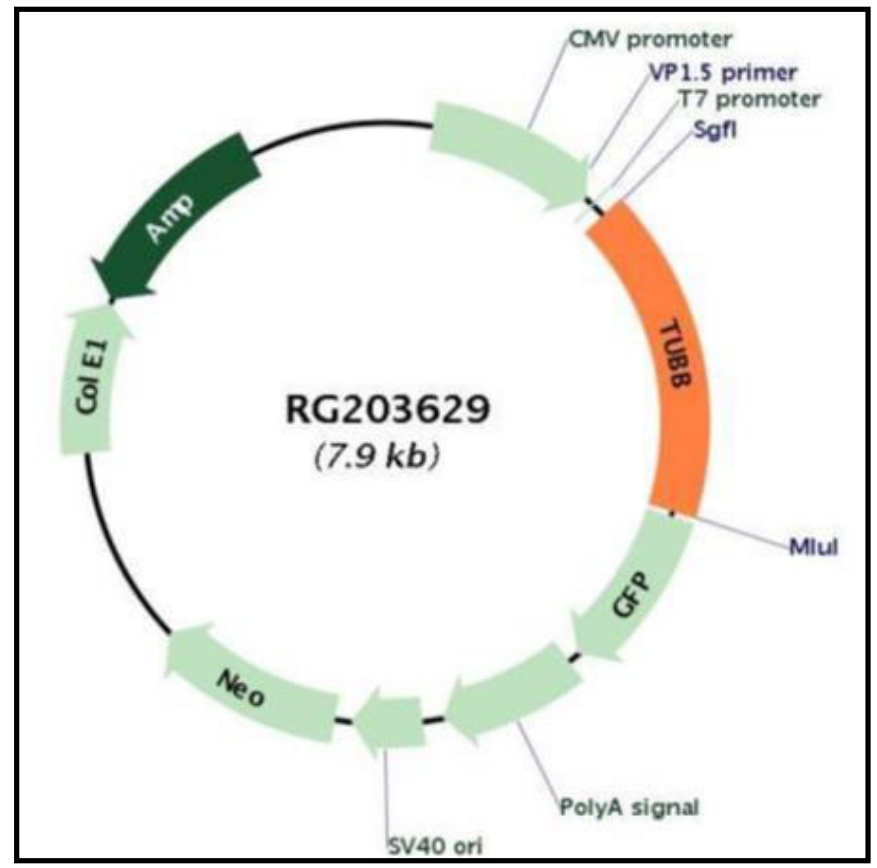

Figure 2.3.1. Expression vector for expression of mutant $\beta$ I-tubulin. Schematic showing the PrecisionShuttle plasmid vector used for transfection of mutant human $\beta I$-tubulin (TUBB). The genomic sequences at the top of the panel are highlighted in red to represent sites for digest by restriction enzymes $\mathrm{SgF} 1$ and Mlu 1 for cloning into cassette. Sequence between these regions encodes human $\beta$ I-tubulin with K124A (Lys124Ala) and E127A (Glu127Ala) substitutions highlighted in blue.

\subsubsection{Transfection optimisation}

HEK293 cells were seeded in 12 -well plates at $2 \times 10^{5}$ cells per well in $1 \mathrm{~mL}$ of antibioticfree DMEM and incubated overnight. At $75-80 \%$ confluence, transfection was carried out using different quantities of wild-type plasmid DNA and Lipofectamine $3000^{\circledR}$ transfection reagent (Invitrogen) to determine the optimum conditions for each. Three different amounts of DNA $(0.5,0.75$ and $1.0 \mu \mathrm{g})$ were trialled by diluting the plasmid solution with P3000 ${ }^{\circledR}$ reagent (Invitrogen) in a 1:2 ratio (DNA:P3000 ${ }^{\circledR}$ ). These mixtures were diluted to a final volume of $50 \mu \mathrm{L}$ per well in serum and antibiotic-free DMEM. The ratio of $\mu \mathrm{g}$ DNA to $\mu \mathrm{L}$ Lipofectamine $3000^{\circledR}$ was varied between $1: 2,1: 3$ and 1:4 for each of the different DNA amounts. This was done by diluting the transfection reagent in a separate tube to a final volume of $50 \mu \mathrm{L}$ with serum and antibiotic-free DMEM and vortexing gently for 2-3 sec. Each DNA/P3000 ${ }^{\circledR}$ solution was then added to the corresponding Lipofectamine $3000^{\circledR}$ concentration and incubated at room temperature (RT) for 10 min to allow for the formation 
of cationic-lipid complexes. Transfection mixture $(100 \mu \mathrm{L})$ was then added to wells in duplicate to give a final volume of $1 \mathrm{~mL}$ medium. Cells were subsequently grown for $48 \mathrm{~h}$ and assessed for GFP expression and cell viability using flow cytometry as described in section 2.3.7. Prior to this, cells were also imaged using the $10 \mathrm{x}$ objective on an Olympus IXS1 fluorescence microscope (Olympus, Tokyo, Japan) under the FITC filter and a mercury lamp excitation source. The same field of view was also imaged with phase-contrast light microscopy.

\subsubsection{Transfection for subsequent experiments}

HEK293 cells were seeded in 6-well plates at a density of $5 \times 10^{5}$ cells per well in $2 \mathrm{~mL}$ of antibiotic-free DMEM and incubated overnight. At $75-80 \%$ confluence, transfection was carried out in the same manner as described in section 2.3.4 using the pre-determined optimal conditions. To this end, $2 \mu \mathrm{g}$ per well of wild-type, D297I, E127A or K124A $\beta I-t u b u l i n$ plasmid DNA was combined with $4 \mu \mathrm{L}$ per well of $\mathrm{P} 3000^{\circledR}$ reagent and diluted to a final volume of $125 \mu \mathrm{L}$ per well in antibiotic and serum-free DMEM. Lipofectamine $3000^{\circledR}(6 \mu \mathrm{L})$ was then diluted to $125 \mu \mathrm{L}$ per well with antibiotic and serum-free DMEM in a separate tube and vortexed gently for 2-3 seconds. The DNA/P $3000^{\circledR}$ solution was then combined with the diluted Lipofectamine $3000^{\circledR}$, mixed gently and allowed to incubate at RT for $10 \mathrm{~min}$. After this time, $250 \mu \mathrm{L}$ of transfection complex was added to each corresponding well to give a final volume in each well of $2 \mathrm{~mL}$. Cells were incubated at $37^{\circ} \mathrm{C}$ for $48 \mathrm{~h}$ prior to use to ensure maximal and consistent expression of the exogenous protein. Transfection efficiency was also determined at this point using fluorescence microscopy and flow cytometry as outlined in sections 2.3 .4 and 2.3.7, respectively.

\subsubsection{Quantification of transfection efficiency and cell viability by flow cytometry}

Two days after transfection for either optimisation of conditions or the subsequent experiments, cell viability and/or transfection efficiency was measured by flow cytometry. At this time cells from each well in either 12- or 6-well plates were harvested with TrypLE ${ }^{\mathrm{TM}}$ and centrifuged. The cell pellet was then washed by resuspension in PBS and centrifugation. Cells were re-suspended in FACS buffer $\left(2 \% \mathrm{FCS}, 0.1 \% \mathrm{NaN}_{3}\right)$ and transferred to roundbottom polystyrene tubes (Fisher Scientific). The tubes were kept on ice and in the dark until 
analysis to prevent GFP bleaching and loss of fluorescence signal. If cell viability was to be assessed, as in section 2.3.4, propidium iodide (PI, Sigma-Aldrich) was added at this step from a stock solution of $1 \mathrm{mg} / \mathrm{mL}$ to give a final concentration of $50 \mu \mathrm{g} / \mathrm{mL}$ and incubated on ice for $5 \mathrm{~min}$. Cell samples were analysed using a BD FACSCanto ${ }^{\mathrm{TM}}$ II flow cytometer (BD Biosciences). For each sample 10,000 events were counted and were subsequently analysed with FlowJo v10.1r5 software (FlowJo, Ashland, OR, USA). Cells were grouped into positive or negative for PI fluorescence, with those negative then gated for GFP signal. The percentage of cells in the counted population that were alive and fluorescent for GFP were then calculated and compared.

\subsubsection{Quantification of exogenous tubulin expression by western blotting}

Samples of HEK293 cells were analysed 72 and 144 hours post-transfection for the MTT cell proliferation assay (section 3.3.1) at the time of fixation for the cell cycle assay (section 3.3.2). Whole cell samples containing approximately $2 \times 10^{6}$ cells were collected in each case and centrifuged at $400 \mathrm{~g}$ for $5 \mathrm{~min}$ in $1.5 \mathrm{~mL}$ Eppendorf tubes. The resulting supernatant was aspirated and the pellets immediately stored at $-80^{\circ} \mathrm{C}$. Following the completion of the aforementioned assays cell lysates were prepared from the frozen samples by addition of 60 $\mu \mathrm{L}$ RIPA buffer (25 mM Tris-HCl, $150 \mathrm{mM} \mathrm{NaCl}, 5 \mathrm{mM}$ EDTA, 0.1\% SDS, $1 \%$ Triton X100, $1 \%$ sodium deoxycholate) with $10 \mu \mathrm{L} / \mathrm{mL}$ Protease Inhibitor Cocktail I (Sigma-Aldrich) added immediately prior. Pellets were suspended in this solution by vortexing and mixed with an orbital shaker for $30 \mathrm{~min}$ at $4^{\circ} \mathrm{C}$ to enable total lysis and maximum dissolution of cellular protein. The samples were then centrifuged at $14,000 \mathrm{~g}$ for $20 \mathrm{~min}$ at $4^{\circ} \mathrm{C}$. The supernatant was transferred to new $1.5 \mathrm{~mL}$ Eppendorf tubes as it contained the desired tubulin protein, while the remaining pellets were discarded. For the following steps sample protein solutions were kept on ice. At this point the BCA Protein Assay (Thermofisher) was used to quantify the concentration of protein present in each sample following the manufacturer's protocol. Samples were then prepared for electrophoretic separation by SDS-PAGE. This was done by diluting $30 \mu \mathrm{g}$ of protein in $1 \mathrm{x}$ Laemmli buffer (4.0 M SDS, 6.1 M glycerol, 0.22 M Tris$\mathrm{HCl}(\mathrm{pH}$ 6.8), $0.75 \mathrm{mM}$ bromophenol blue) containing 10\% $\beta$-mercaptoethanol and Milli-Q water to make up $20 \mu \mathrm{L}$ of sample. Each lane of a $1.5 \mathrm{~mm} 10 \%$ polyacrylamide resolving gel with a $4 \%$ stacking gel was loaded with $20 \mu \mathrm{L}$ of sample. In addition, $1.5 \mu \mathrm{L}$ of a molecular

weight ladder (Dual Colour Precision Plus ${ }^{\mathrm{TM}}$, Bio-Rad Laboratories, Auckland, New Zealand) 
was added to a lane in order to identify the proteins of interest based on size. The remaining cell lysates were stored at $-80^{\circ} \mathrm{C}$ until analysis. Separation was achieved by running the gel for $90 \mathrm{~min}$ at $120 \mathrm{~V}$ or until the dye front had reached the end of the gel. Proteins within the gel were transferred to a methanol-activated low fluorescence background PVDF membrane (Bio-Rad Laboratories) via western blot for $17 \mathrm{~h}$ at $20 \mathrm{~V}$ at $4^{\circ} \mathrm{C}$. The membrane was then blocked with 5\% BSA in TBS-T ( 1 x TBS, $0.1 \%$ Tween-20) for $1 \mathrm{~h}$, followed by three 10 min washes with TBS-T, all done at RT temperature on a rocking platform. Acrylamide gels were also stained with Coomassie Blue for protein that did not transfer at this point. They were treated with fixation solution (50\% ethanol, 3\% ortho-phosphoric acid in Milli-Q water) for $1 \mathrm{~h}$ at RT and washed three times with Milli-Q water. They were then stained with Coomassie Blue solution (0.006\% Coomassie ${ }^{\circledR}$ Brilliant blue G-250 (Bio-rad Laboratories) in $34 \%$ methanol, 17\% ammonium sulphate, $3 \%$ phosphoric acid) and left on a rocking platform at RT for two days prior to assessment of transfer success. Washed membranes were probed immunologically by adding $4 \mathrm{~mL}$ of $1 \% \mathrm{BSA}$ in TBS-T containing the primary monoclonal mouse antibodies for $\beta$-tubulin (IgG1, clone AA2, Sigma-Aldrich) and $\beta$-actin (IgG2a, clone AC-74, Sigma-Aldrich), both in a 1:1000 dilution in the same solution, to each membrane. The membranes were left on a rocking platform at $4{ }^{\circ} \mathrm{C}$ overnight and then washed three times for $10 \mathrm{~min}$ with TBS-T. Four $\mathrm{mL}$ of $1 \%$ BSA in TBS-T containing a goat anti-mouse IgG $(\mathrm{H}+\mathrm{L})$ secondary antibody conjugated to an Alexa Fluor ${ }^{\circledR} 488$ fluorophore (ThermoFisher) in a 1:2000 dilution was next added to each membrane, which was left on a rocking platform for $1 \mathrm{~h}$ in the dark. This was followed by another round of three $10 \mathrm{~min}$ washes, this time with TBS alone. Immediately after the final wash, the membranes were scanned with a Typhoon ${ }^{\mathrm{TM}}$ 9500 laser scanner (GE Healthcare Life Sciences, Auckland, New Zealand), and images were generated using the accompanying software. Image densitometry was carried out with ImageJ software (NIH) to quantify the relative density of detected bands corresponding to the proteins of interest, adjusting for background fluorescence and normalising against the band density of the loading control $\beta$-actin.

\subsubsection{Assessment of microtubule morphology and exogenous tubulin expression by fixed-} cell confocal microscopy

To increase the adherence of cells to glass coverslips used in confocal microscopy, they were first treated with Histogrip $^{\text {TM }}$ (ThermoFisher) as per the manufacturer's instructions. HEK293 
cells seeded onto coverslips placed in 6-well plates were transfected with either wild-type, D297I, E127A or K124A mutant $\beta$ I-tubulin, or mock-transfected. After $48 \mathrm{~h}$ they were fixed in paraformaldehyde (PFA) by incubating coverslips in $1 \mathrm{~mL}$ fixative solution (4\% PFA in PBS, pH 6.9) for 20 min on a rocking platform at RT. From this point onwards all processing of coverslips was carried out in the dark so as to reduce bleaching of the GFP signal. The fixative was then removed and the coverslips washed three times with PBS-T (1x PBS, 0.1\% Tweem-20) for 5 min each on a rocking platform at RT. They were then blocked with $5 \%$ BSA in PBS-T at RT on a rocking platform for $1 \mathrm{~h}$. Three further 5 min washes with PBS-T were carried out prior to immunocytochemistry staining. This was done by incubating coverslips in $1 \mathrm{~mL}$ of $5 \%$ BSA in PBS-T containing monoclonal anti- $\beta$-tubulin mouse primary antibody (Sigma-Aldrich) diluted to 1:1000 at RT on a rocking platform for $2 \mathrm{~h}$. Coverslips were washed three times with PBS-T. $1 \mathrm{~mL}$ of $1 \%$ BSA in TBS-T containing a goat anti-mouse IgG secondary antibody conjugated to Cy3 fluorophore (ThermoFisher) in a 1:2000 dilution was next added to each coverslip, which was left on a rocking platform at RT for $1 \mathrm{~h}$ in the dark. A further three 5 min washes were then carried out with PBS alone and the coverslips were mounted on microscope slides using 46 Gold Antifade ${ }^{\circledR}$ with 4', 6deamidine-2'-phenylindole (DAPI, Invitrogen) in order to stain the nuclei of cells. Slides were stored in the dark at $4^{\circ} \mathrm{C}$ until imaging. This was done with an Olympus FluoView FV1000 confocal laser-scanning microscope using a 100x oil immersion objective. A $405 \mathrm{~nm}$ laser was used to image the DAPI-bound nuclei, Cy3-labelled $\beta$-tubulin was with a $559 \mathrm{~nm}$ laser, while a $473 \mathrm{~nm}$ laser was used to excite exogenous $\beta$ I-tubulin-bound GFP.

\section{$2.4 \quad$ Results}

\subsubsection{Quantification and evaluation of wild-type and mutant $\beta I$-tubulin plasmid DNA}

The wild-type and D297I, E127A and K124A mutant $\beta$ I-tubulin expression vectors were prepared as described in section 2.2.3 and were analysed for DNA concentration by spectrophotometry. The concentrations for each of the preparations, taken as the mean of three consecutive measurements, were found to be $617 \pm 14 \mathrm{ng} / \mu \mathrm{L}$ for wild-type, $901 \pm 12$ $\mathrm{ng} / \mu \mathrm{L}$ for D297I, $752 \pm 10 \mathrm{ng} / \mu \mathrm{L}$ for E127A and $1141 \pm 26 \mathrm{ng} / \mu \mathrm{L}$ for K124A. All four plasmids gave high mean A260/A280 and A260/A230 ratios of 1.8-1.9 and 2.39-2.48, respectively. This suggests that they contained very low levels of protein or solvent contaminants used in the isolation and purification procedure, an important quality required 
of DNA to be used for transfection experiments to reduce target cell toxicity. Therefore, all plasmid preparations were suitable for use in the subsequent assays.

\subsubsection{Optimisation of transfection conditions}

HEK293 cells were transfected with wild-type $\beta$ I-tubulin plasmid in nine different condition combinations of DNA concentration $(0.5,0.75$ and $1.0 \mu \mathrm{g})$ and ratio of $\mu \mathrm{L}$ Lipofectamine $3000^{\circledR}$ to $\mu \mathrm{g}$ DNA $(2: 1,3: 1$ and $4: 1)$. These were then assessed and analysed with flow cytometry and fluorescence microscopy to determine which gave the highest rate of successful transfection while maintaining low levels of cell toxicity. For the flow cytometric analysis, transfection efficiency was measured as the percentage of cells in each sample that were positive for GFP fluorescence. Cell death was determined by those positive for PI fluorescence. PI is a membrane-impermeable nucleic acid stain, and will therefore only fluoresce in dead cells with compromised membrane integrity. An issue with analysing cells with both GFP and PI signals is that their emission spectra overlap between $550 \mathrm{~nm}$ and 650 nm wavelengths. Therefore, compensation parameters were applied to correct for any crosschannel leakage to enable discrete fluorescence detection of GFP in the presence of PI.

Figure 2.4.1 shows the gating strategy employed for the flow cytometric analysis of transfection efficiency. First, cells were plotted on a PI-area histogram, giving a major and minor peak corresponding to live and dead cells, respectively. The live cells were then plotted as forward scatter vs. side scatter and gated for single cells. These were plotted on a histogram of GFP-area, gating for GFP negative and positive cells to determine the percentages of each. 
A

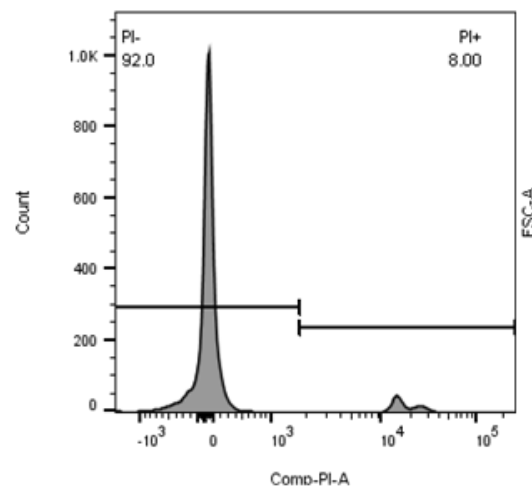

B

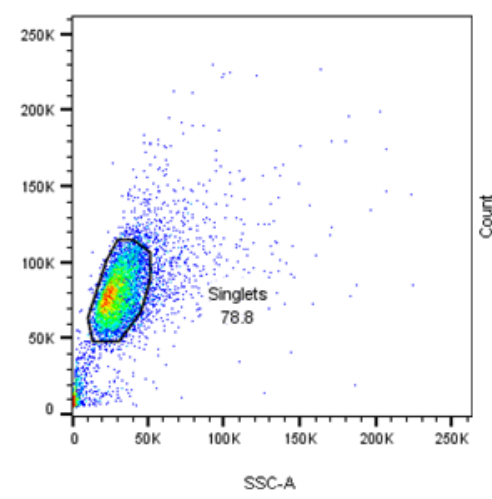

C

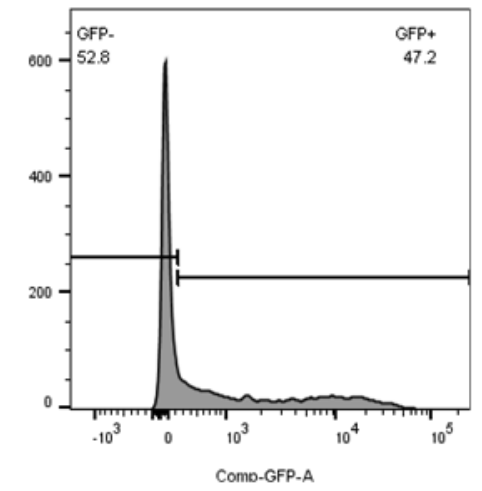

Figure 2.4.1. Flow cytometry workflow for transfection efficiency and cell death analysis. This figure shows flow cytometry results from one transfected cell sample to demonstrate typical analytical workflow. (A) PI-area histogram showing PI- and PI+ events. (B) Forward scatter (y-axis) vs. side scatter (x-axis) for PI- (live) cells gated for singlets. (C) GFP-area histogram of single cells showing GFP- and GFP+ events.

The results of the optimisation experiment are shown in Figure 2.4.2. Transfection with 0.5 $\mu \mathrm{g}$ DNA produced the lowest numbers of GFP-fluorescent cells, as did $0.75 \mu \mathrm{g}$, both at a Lipofectamine:DNA ratio of $2: 1$, with mean efficiencies of $11-16 \%$ of the total cell population. The combination that gave the highest mean transfection efficiency $(53 \pm 3 \%)$ was $1.0 \mu \mathrm{g}$ plasmid mixed with Lipofectamine $3000^{\circledR}$ in a $3: 1$ ratio. Upon inspection of transfected cells by fluorescent microscopy all cell cultures showed low numbers of dead cells, identified as rounded, floating cells or cell fragments. The largely well-maintained adherent morphologies with few dead cells, suggested a lack of toxicity caused by the procedure (Figure 2.4.3). This is supported by the low numbers of dead cells as determined directly by positive PI staining. While the conditions that gave the greatest transfection efficiency also resulted in the highest mean percentage dead cells $(12 \pm 3 \%)$, all replicates of each combination showed less than $15 \%$ of the total cell populations had died. Due to these low dead cell numbers and the lack of major morphological changes in the viable cells, as well as the high efficiency results, $1.0 \mu \mathrm{g} / 3: 1$ was chosen to be used in all subsequent experiments. This was scaled up for the transfection of cells seeded in 6-well plates. Specifically, $2.0 \mu \mathrm{g}$ plasmid was mixed with $6 \mu \mathrm{L}$ Lipofectamine $3000^{\circledR}$ per well. 
Transfection Optimisation

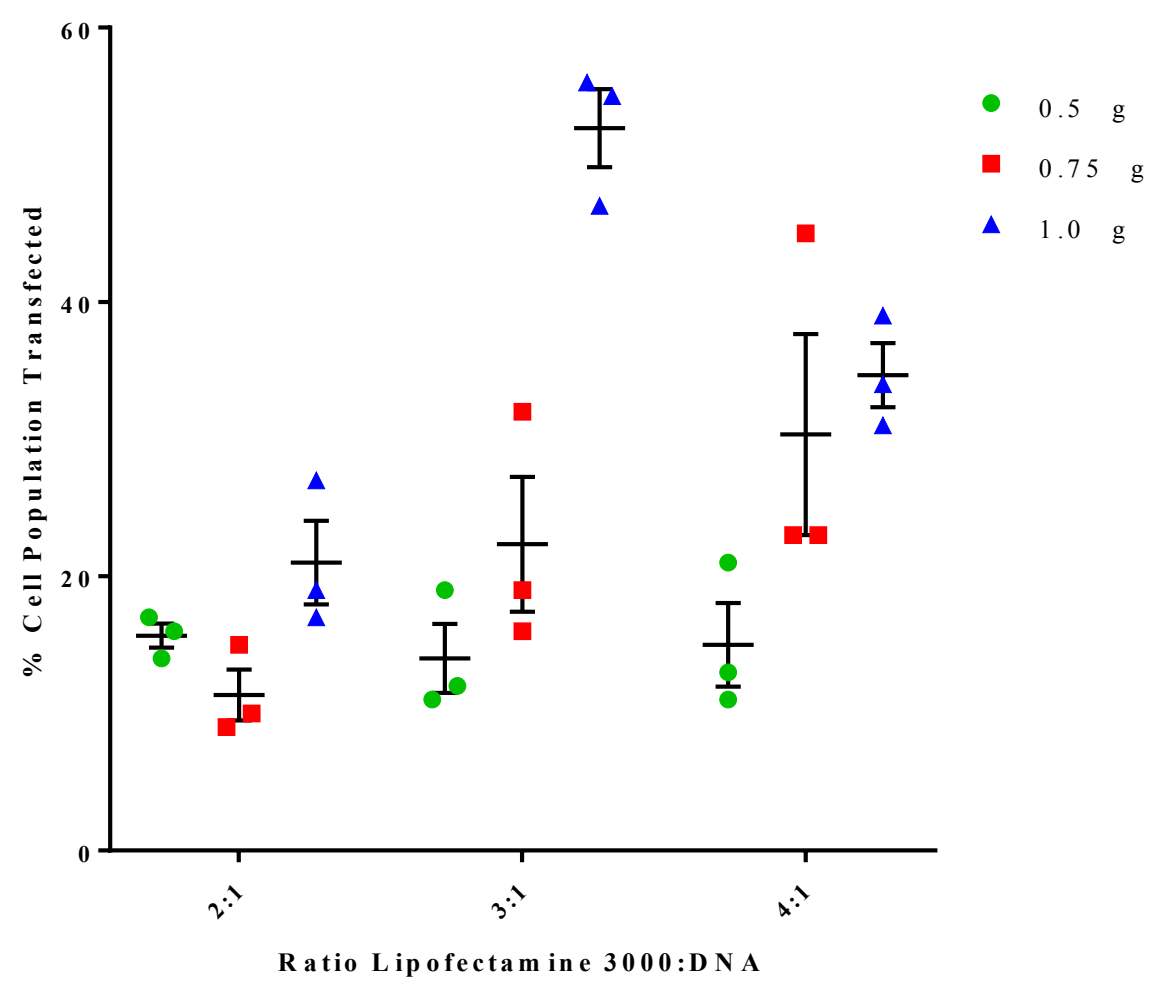

Figure 2.4.2. Transfection efficiencies during optimisation by flow cytometry. Plot shows the percentage of the live cell population positive for GFP fluorescence in each sample transfected with a different reagent/DNA combination. HEK293 cells transfected with wildtype $\beta$ I-tubulin plasmid were used in this optimisation experiment and the different optimisation conditions are shown on the $\mathrm{x}$-axis and in the legend. Data presented as mean \pm $\operatorname{SEM}(n=3)$. 


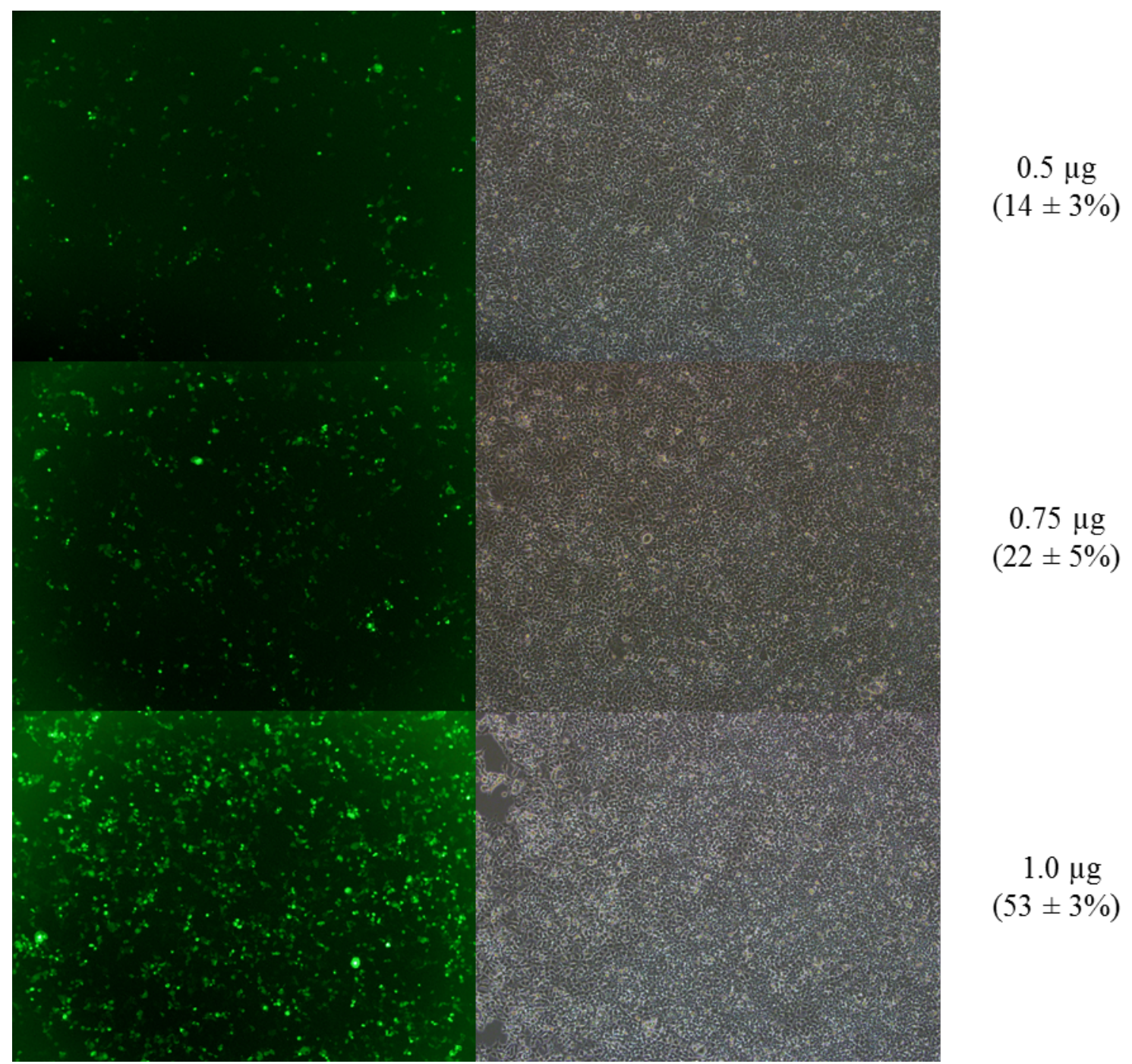

Figure 2.4.3. Assessment of transfection optimisation by fluorescent microscopy. Images show cells transfected with Lipofectamine $3000^{\circledR}$ in a $3: 1$ ratio with three different concentrations of wild-type $\beta I$-tubulin plasmid. Images on the left were captured using the FITC filter set with GFP excited by a mercury lamp. Images on the right represent the same field of view under phase-contrast. All were taken using the 10x magnification objective. Values in brackets are mean transfection efficiencies as determined by flow cytometry from 3 biological replicates, as described in section 2.4 .3 below.

\subsubsection{Transfection efficiencies measured by flow cytometry for MTT assays}

Samples of cells transfected with wild-type and D297I, E127A and K122A mutant $\beta$ I-tubulin were collected at the $48 \mathrm{~h}$ and $144 \mathrm{~h}$ post-transfection time points of each MTT assay and analysed by flow cytometry for GFP fluorescence to estimate the transfection efficiency 
(Figure 2.4.4). There were no significant differences between the percentages of GFPpositive cells for the four different transfection types at each time point $(\mathrm{P}>0.05)$, showing a high degree of consistency in terms of plasmid performance. For each experiment, high mean efficiencies of between $57 \%$ and $68 \%$ were observed $48 \mathrm{~h}$ after plasmid was added. However, for each assay this decreased significantly to between $8 \%$ and $13 \%$ at $144 \mathrm{~h}(\mathrm{P}>0.01)$.

\section{Transfection Efficiency During M T T Assays}

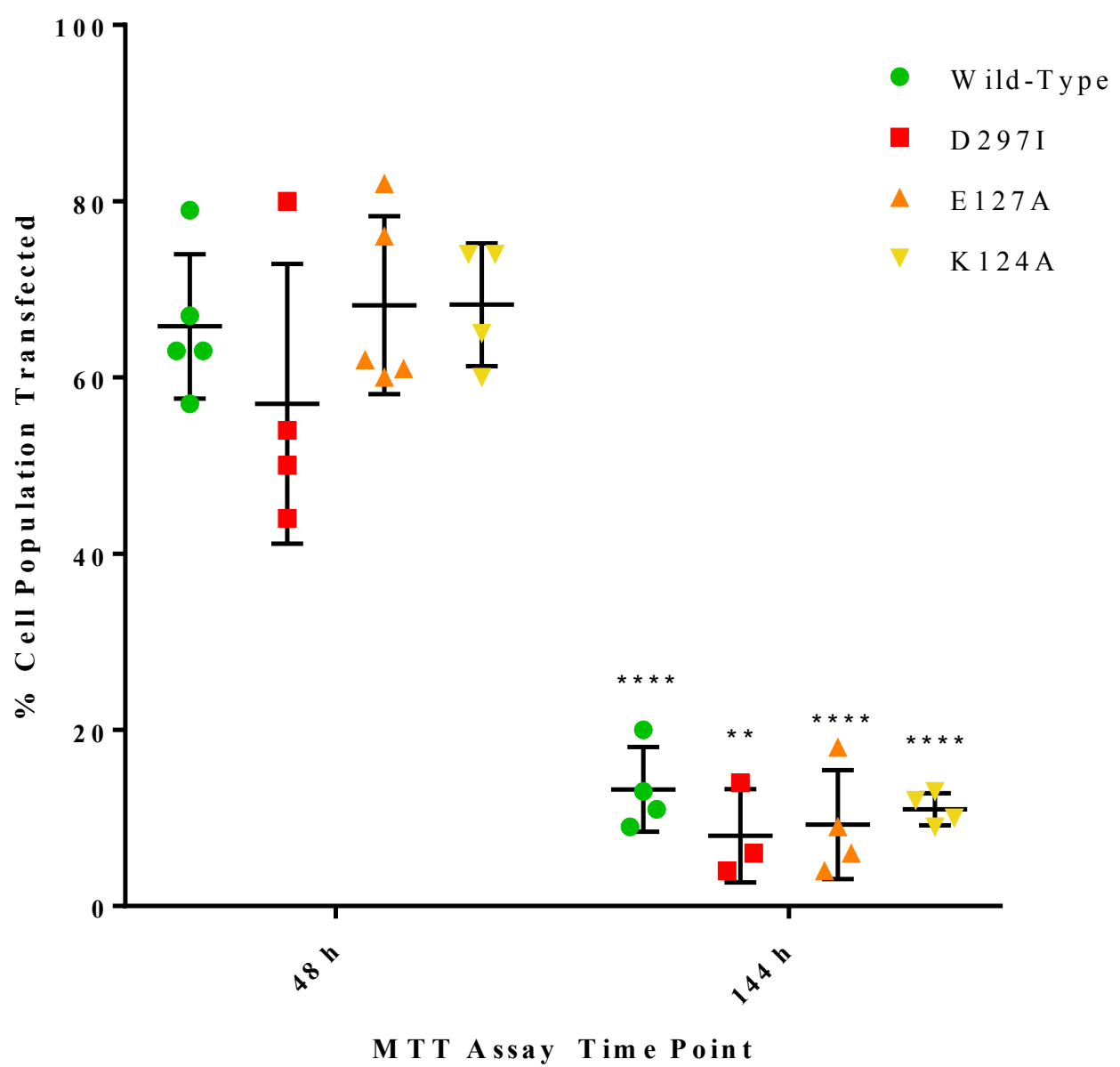

Figure 2.4.4. Transfection efficiencies during MTT assays. Graph shows the percentage of the total cell population that is positive for GFP fluorescence in samples transfected with each of the four $\beta$ I-tubulin plasmids. Samples correspond to post-transfection time points at the start $(48 \mathrm{~h})$ and finish $(144 \mathrm{~h})$ of the MTT assay. Data presented as mean \pm SEM $(\mathrm{n}=4-5)$. $* *=\mathrm{P}<0.01, * * * *=\mathrm{P}<0.0001$ vs. 48 h by Student's t-test.

This loss in transfection efficiency was investigated further by transfecting cells that had been seeded in 6-well plates with either 1.5 or $2 \mu \mathrm{g}$ of wild-type $\beta \mathrm{I}$-tubulin plasmid and measuring 
the efficiency each day for six days following transfection (Figure 2.4.5). The results showed for both DNA concentrations that the peak number of cells positive for GFP was reached by day 2, after which time this gradually decreased to similar levels as for the MTT assay experiments of Figure 2.4.4. This is important as the number of cells expressing the exogenous $\beta I$-tubulin throughout the course of the MTT assays will impact on the degree of change to drug response that the mutations may impart. However, as reducing the amount of DNA used did not improve the loss of efficiency, but rather reduced it at each time point, the transfection protocol was not modified.

Transfection Efficiency Time Course

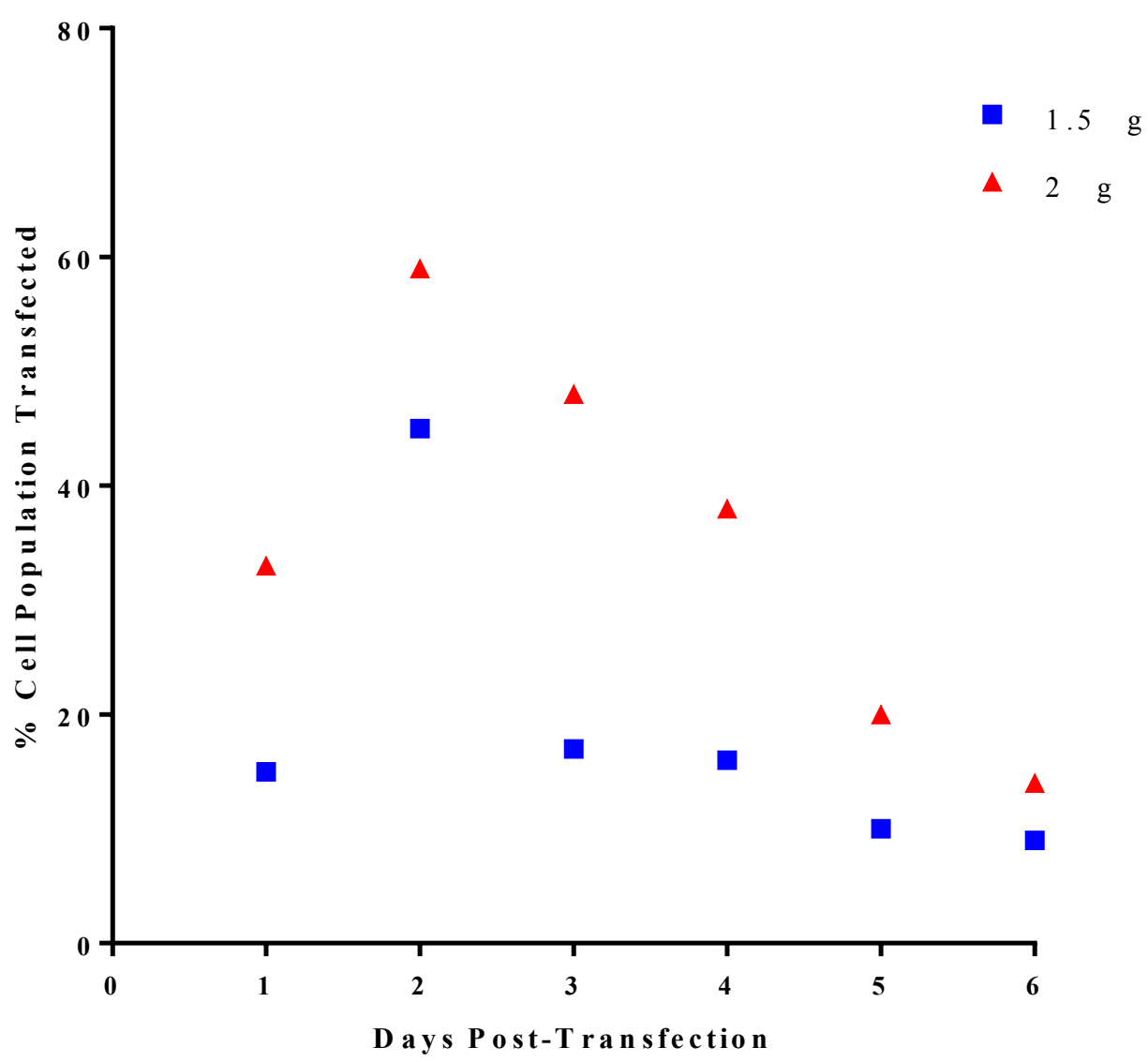

Figure 2.4.5. Investigation of changes in transfection efficiency over 6 day course of a typical MTT assay. Graph shows the percentage of the total cell population positive for GFP fluorescence in samples transfected with wild-type $\beta$ I tubulin plasmid at two different DNA concentrations, $1.5 \mu \mathrm{g}$ and $2 \mu \mathrm{g}$. Fluorescent cell counts were measured by flow cytometry each day after plasmid was added for six days $(n=1)$. 


\subsubsection{Transfection efficiencies measured by western blotting for MTT and cell cycle assays}

As well as determining the percentage of cells within a population successfully transfected, it is also necessary to understand what proportion of total $\beta$-tubulin protein is made up of GFPtagged exogenous subunits. As described in section 2.3.8, western blotting was used to measure the relative abundance of both endogenous and exogenous $\beta$-tubulin subunits within transfected cell populations used for both MTT and cell cycle assays.

Unfortunately, none of the experiments gave strong, defensible bands corresponding to native $\beta$-tubulin at $50 \mathrm{kDa}$ or GFP-tagged $\beta$-tubulin at $75 \mathrm{kDa}$ (figure 2.4.6). There was no issue with protein loading as demonstrated by the intense bands for the ubiquitous protein $\beta$-actin seen at $42 \mathrm{kDa}$. Nor were there concerns of incomplete transfer as staining of gels indicated no remaining proteins in acrylamide gels. While faint bands do appear to be present at 75 $\mathrm{kDa}$ and another below, these cannot be defined as the proteins of interest due to the size of the lower band being above $50 \mathrm{kDa}$ and the presence of a $75 \mathrm{kDa}$ band for mock-transfected control. Instead, these are likely degradation products or artefacts of non-specific antibody binding. Other such bands can also be seen further down the lanes with strong bands at the very bottom. This suggests that degradation of $\beta$-tubulin, perhaps during sample storage or preparation, may have led to the absence of discernible bands. Another possible contributing factor could be the inclusion of SDS in the transfer buffer as this may inhibit binding of proteins to the membrane during transfer. This may also explain the poorly defined protein ladder bands. 


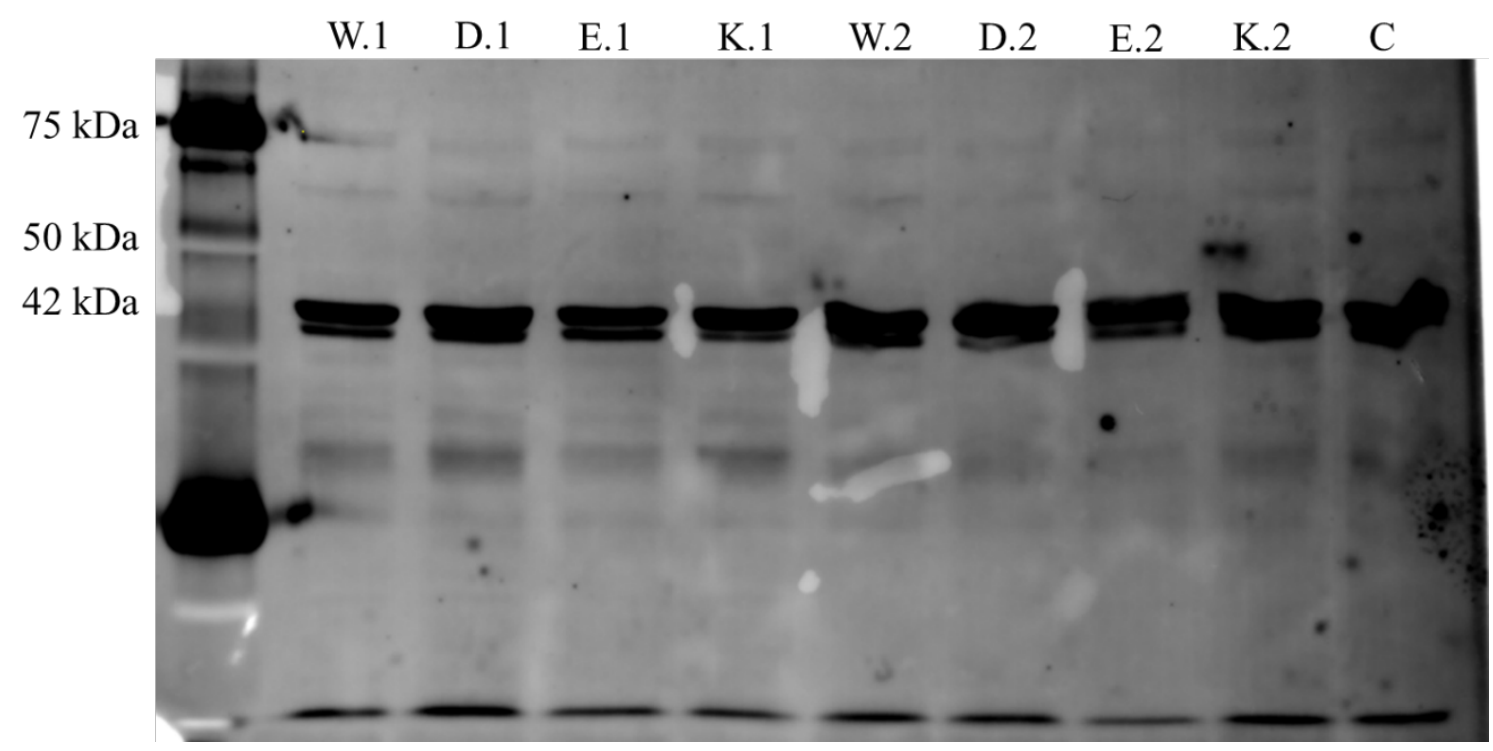

Figure 2.4.6. Western blot of samples from one MTT assay. Image shows immunolabelled membrane from one MTT assay, stained for $\beta$-tubulin (50 kDa native, $75 \mathrm{kDa}$ GFP-tagged) and $\beta$-actin $(42 \mathrm{kDa})$. Lanes labelled based on transfection category and time point i.e. $\mathrm{W}=$ wild-type, $\mathrm{D}=\mathrm{D} 297 \mathrm{I}, \mathrm{E}=\mathrm{E} 127 \mathrm{~A}, \mathrm{~K}=\mathrm{K} 124 \mathrm{~A}, \mathrm{C}=$ mock-transfected control. $1=48 \mathrm{~h}$ posttransfection, $2=144 \mathrm{~h}$ post-transfection. The protein molecular weight ladder is shown on the left. Dark bands at $42 \mathrm{kDa}$ represent $\beta$-actin loading control.

While the amount GFP-tagged protein as a proportion of the total $\beta$-tubulin was able to be used from this western blotting procedure, some evidence was seen in the later in situ polymerisation experiment described in section 3.3.3. For one particular blot using wild-type transfected cells faint bands were observed at both $50 \mathrm{kDa}$ and $75 \mathrm{kDa}$, corresponding to endogenous and GFP-tagged $\beta$-tubulin, respectively (Figure 2.4.7). However, as samples had been separated into soluble and insoluble (polymerised) protein fractions and substantial sample degradation appeared to occur, measurement of introduced tubulin expression was not able to be carried out directly. Despite this, combining band intensities for the two fractions and expressing as a percentage of total intensity gave a wild-type transfection efficiency of $47 \%$. While this merely represents a single wild-type transfected sample collected $64 \mathrm{~h}$ after addition of plasmid, no such analysis was able to be done for any mutant $\beta I$-tubulin transfected cells, nor for later time points important for the MTT assays. This does give some indication that introduced $\beta I$-tubulin subunits are being substantially expressed in successfully transfected cells. However, this would have to be extensively validated by further western blots before any conclusions are drawn. 


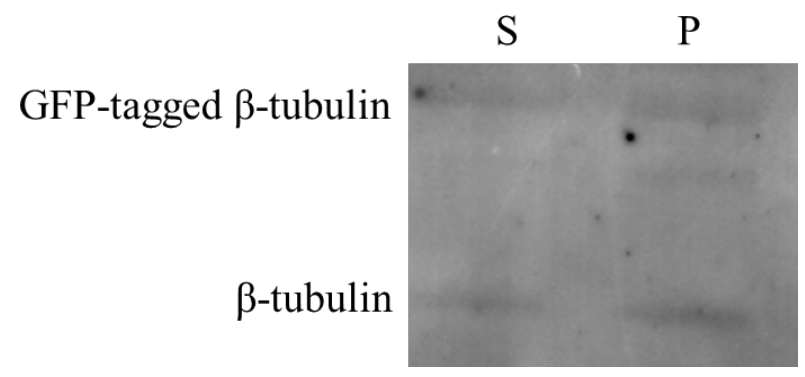

Figure 2.4.7. $\beta$-tubulin bands in in situ polymerisation western blot. Image shows faint bands corresponding to exogenous and native $\beta$-tubulin seen on a membrane for the in situ polymerisation experiment. Lanes are labelled based on tubulin fraction type i.e. $\mathrm{S}=$ soluble, $\mathrm{P}=$ polymerised.

2.4.5. Mutant $\beta$ I-tubulin expression and microtubule morphology examination by immunocytochemistry and confocal microscopy

In order to relate the results seen in this study to the functional impact of introducing the E127A, K124A and D297I point mutations, as well as GFP-tagged wild-type $\beta I-t u b u l i n$, exogenous subunits must successfully integrate into the microtubule macrostructure. This was assessed by fixed cell confocal microscopy and immunocytochemistry, staining for $\beta$ tubulin while also visualising GFP concurrently (Figure 2.4.8). HEK293 cells transfected with either of the mutations or wild-type showed normal polymerised microtubule morphology, as compared with mock-transfected control cells. Successfully transfected cells showed strong, linear GFP signals overlaying the Cy3-labelled $\beta$-tubulin. This suggests that in these cells GFP fusion-tagged exogenous $\beta I$-subunits have been incorporated into the microtubule polymer. However, lightly punctate GFP signal is exhibited for some cells, suggesting a degree of GFP aggregation. 


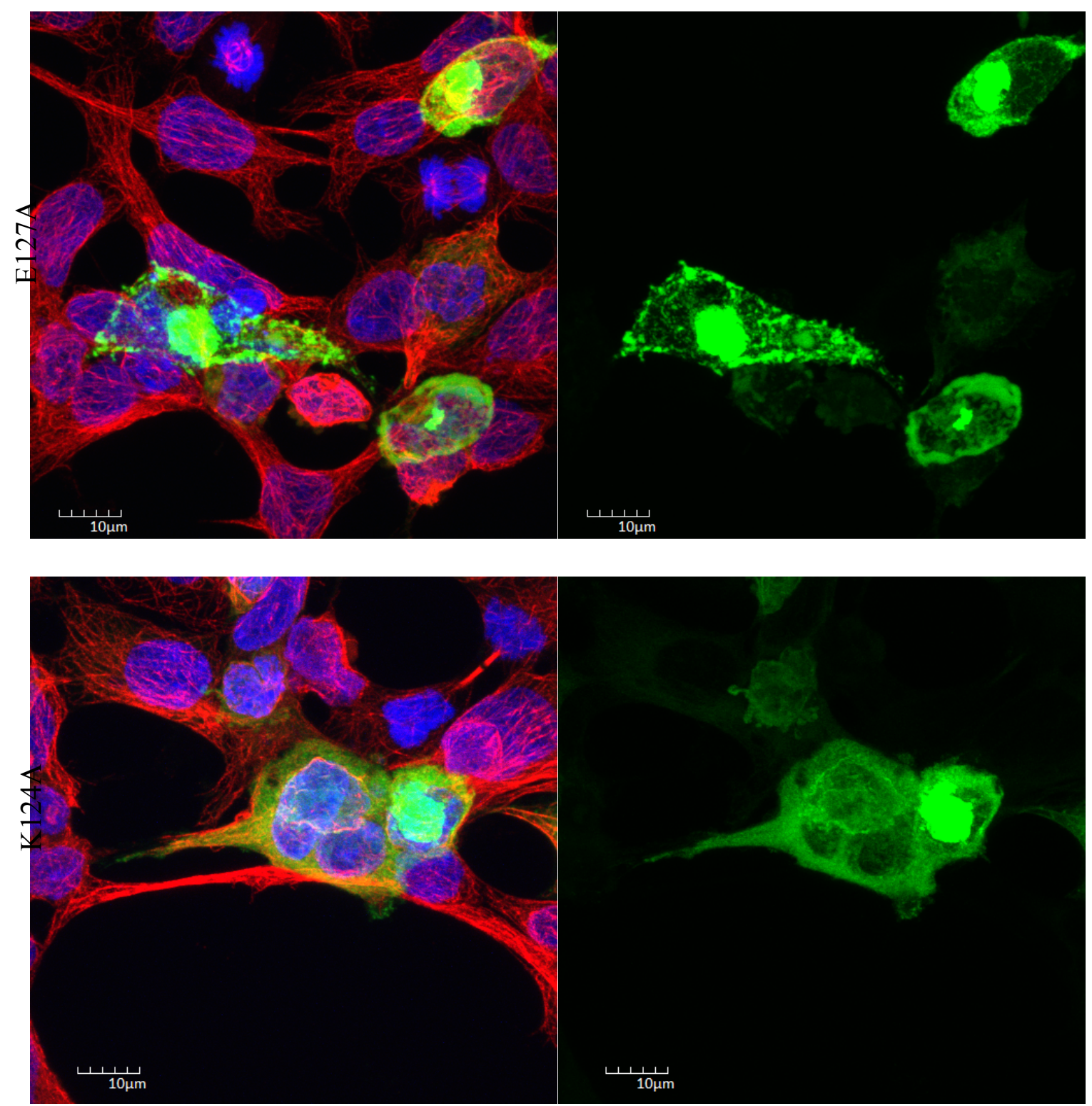

Figure 2.4.8. Confocal microscopy images of transfected HEK293 cells stained for $\beta$ tubulin. Z-stacked images show representative cells from mock-transfected control, wildtype and mutant cell cultures. Cells are fixed onto glass coverslips pre-treated with Histogrip ${ }^{\circledR}$ and stained with Cy3-labelled antibody against $\beta$-tubulin (red signal) and the DNA stain DAPI (blue signal). Side-by-side images show composite images of all fluorescent channels on the left, and GFP signal only on the right. Images captured using the 100x oil immersion lens. Control image shows normal microtubule morphology, which is mimicked well by the GFP-tagged subunit-containing microtubules fluorescing green in the wild-type, D297I and K124A images. E127A mutant transfected cells show a lack of normal morphology for microtubules containing exogenous $\beta \mathrm{I}$-tubulin and the presence of perfunctory GFP aggregates. 


\subsection{Discussion}

\subsubsection{Expression of exogenous mutant $\beta I-t u b u l i n$}

The work outlined in this chapter details the effort to successfully introduce exogenous E127A and K124A mutant $\beta$ I-tubulin in the HEK293 mammalian cell model used. The optimisation of a number of key parameters relating to this transfection procedure is essential in affording inferential power to the subsequent drug response experiments. These include maximising the total number of cells expressing exogenous tubulin, as well as mutant protein expression levels within the transfected cells, ensuring successful incorporation of these subunits into functional microtubule polymers and minimising transfection-induced toxicity towards cells. For example, in ideal circumstances, mutant $\beta I$-tubulin subunits would account for all $\beta I$-tubulin protein within transfected cells. In this case, if either of the mutations altered the binding interactions between PLA or LAU and microtubules this would translate to large, easily distinguishable changes in drug response.

Initially, the 1A9 ovarian cancer cell line was chosen as the desired cell model for this investigation based on its use in previous PLA and LAU mechanistic studies. ${ }^{73}$ However, pilot transfection attempts yielded transfection efficiencies below $10 \%$ by flow cytometry (results not shown). In addition, the cell cryo-stock was suspected to be contaminated with mycoplasma. HEK293 cells were therefore used instead due to their ease of transfection and rapidly dividing nature, with a similar proliferative rate to $1 \mathrm{~A} 9$ cells.

Following protocol optimisation, transfection of HEK293 cells with wild-type and mutant $\beta I-$ tubulin plasmid constructs produced consistently high transgene expression rates among cell populations. Approximately $60 \%$ of cells were successfully transfected across all four transfection types $48 \mathrm{~h}$ after transfection as determined by flow cytometric analysis of GFP fluorescence. Unfortunately, this percentage was also consistently and gradually abolished over the course of $144 \mathrm{~h}$. This suggests that exogenous $\beta \mathrm{I}$-tubulin protein was not present and active within cells over the full length of the MTT assays. As the stability of expression levels will impact on any changes to the drug response of transfected cells, this has implications for the interpretation of results and any inferences drawn between mutant tubulin levels and $\mathrm{IC}_{50}$ values.

This loss of expression from in vitro transfection of episomal DNA has been previously shown to result from chromatin remodelling and nuclease activity. ${ }^{78,79}$ This is particularly prevalent with the use of viral promoter sequences, such as the CMV sequence used to drive 
expression of the transgenes in the present study. ${ }^{80,81}$ This effect is also highly variable between different cell types, largely due to differences in plasmid degradation and dilution rates, the latter a direct effect of cellular proliferation. As HEK293 cells undergo relatively rapid cell division, plasmid copy number would likely decrease at a faster rate when compared to cell lines with longer mitotic cycles. Therefore, the cell-specific characteristics of HEK293 cells coupled with the particular transfection vectors used in this investigation may have resulted in the observed loss of exogenous $\beta$ I-tubulin expression efficiency over time.

However, the measure of transfection efficiency determined from flow cytometry only provides information regarding the proportion of cells expressing GFP-tagged $\beta I$-tubulin in a population. It does not indicate how much of the total $\beta$-tubulin protein pool is composed of exogenous subunits, which is vital to the understanding of the relationship between the introduced mutations and MSA activity. Furthermore, as the former measure is based solely on GFP fluorescence, it is possible that loss of signal over time through bleaching by ambient light could also contribute to the results seen and therefore lead to underestimated values. This is another reason why analysis of total protein concentrations is important in the context of this study. In addition, if the introduction of GFP-tagged $\beta I$-tubulin resulted in a reduced proliferative ability compared to mock-transfected cells, the latter could out-grow the former over time, gradually decreasing the percentage of fluorescent cells in a population. While results from subsequent experiments suggest that this may apply to E127A mutant transfected cells, they do not support the loss of efficiency for wild-type cells also.

Western blotting was used to determine the percentage of GFP-tagged $\beta$-tubulin in cells transfected for MTT and cell cycle assays, in parallel with the aforementioned flow cytometric analysis. However, no $\beta$-tubulin bands were observed for any of the blots carried out despite successful protein loading and transfer as indicated by the presence of $\beta$-actin bands and lack of gel staining, respectively. This same protocol has been used successfully in previous studies to determine the efficiency of $\beta$-tubulin transfection procedures in HEK293 cells. ${ }^{74}$ Issues have arisen with a lack of GFP-tagged $\beta$-tubulin, however in these cases native $\beta$-tubulin bands have consistently been observed. Two potential reasons for the absence of bands seen here is the presence of SDS in the transfer buffer used and degradation of tubulin during sample storage or processing. SDS is added to the buffer used during the transfer of proteins from the acrylamide gel to a PVDF membrane to assist in elution from the gel by imparting a negative charge to proteins. However, this in turn can inhibit the binding of many 
proteins, particularly those of smaller molecular weight, to the membrane. In addition, tubulin proteins are very temperature-sensitive, hence sample preparation is carried out on ice and whole cell and lysate samples are stored at $-80^{\circ} \mathrm{C}$. It is therefore possible that improper storage or processing during these experiments has led to protein degradation. This is supported by multiple faint bands seen further down the lanes of several blots, as well as intense bands seen at the bottom of the lanes. Some information regarding exogenous protein levels was gathered from a later in situ polymerisation western blotting experiment that produced bands of molecular weights corresponding to native and GFP-tagged $\beta$-tubulin for a wild-type transfected sample. While not a repeated or defensible measure, it does suggest that the transfection efficiency data gathered may translate to high proportions of exogenous subunits in transfected cells. However, this does not provide any further insight as to the apparent reduction in introduced $\beta$-tubulin over time.

Evidence from previous studies studying the effect of point mutations on MSA activity suggests that even a low transfection efficiency may still reveal a significant change in drug sensitivity if the impact on binding association is large enough. For example, a taxane region point mutation of amino acid L215 in only 5\% of $\beta$ I-tubulin in a particular investigation produced a significant increase in PTX resistnace. ${ }^{82}$ However, the effect was considerably lower than other studies with a greater or complete abundance of mutant target protein. A 1A9 sub-clone, L4, resistant to LAU was developed through prolonged selection in the presence of the compound. ${ }^{83}$ The resulting cells were 39 times more resistant to PLA and LAU than normal 1A9 cells. The point mutations $\mathrm{R} 306 \mathrm{H} / \mathrm{C}$ in $\beta \mathrm{I}$-tubulin were found to be the cause of this change in response. The results of the study suggested that R306 is important to the target association of these PLA and LAU and represents the first cell-based evidence of their shared binding site interactions. A lesser, but still considerable, 6-fold increase in resistance to PLA only was also observed in R1 1A9 clones harbouring the A296T $\beta I-t u b u l i n$ mutation.

In order to draw any conclusions relating the E127A and K124A mutations to PLA or LAU response, the successful incorporation of these subunits into functional microtubules should be confirmed. The imaging of fixed HEK293 cells stained for $\beta$-tubulin and chromosomal DNA was used to this end. GFP signal overlapped well with that of Cy3-labelled $\beta$-tubulin in wild-type and mutant transfected cells, showing that GFP-tagged subunits appeared to form polymerised microtubules. As the introduction of these GFP-tagged subunits has the potential to disrupt normal microtubule dynamics, the functionality of these polymers was assessed. 
Not only did the morphology of microtubules seen by GFP signal align with the $\beta$-tubulin signal of the same cells, they also showed similar morphology to mock-transfected control cells. This suggests that the presence of the GFP tag did not alter the biological function of microtubules in this mammalian cell system. This is also supported by a previous study that employed the same expression plasmid to transfect 1A9 and HEK293 cells with different $\beta$ Itubulin point mutations. Immunocytochemistry was used to visualise GFP signal, observing no alteration in biological function nor the dynamic equilibrium of mutant tubulin-expressing microtubules. $^{74}$

In conclusion, the experiments reported in this chapter have optimised the procedure for the expression of E127A and K124A mutant $\beta$ I-tubulin in HEK293 cells. Despite a lack of evidence for the proportion of exogenous protein expressed in transfected cells, consistently high population efficiency data was gathered from other analyses and is in accordance with previous studies that have utilised similar protocols. Together with the demonstration that GFP-tagged subunits are incorporated successfully into biologically active microtubules, this validates the current system. It was therefore employed in subsequent experiments to investigate the role of glutamate 127 and lysine 124 in PLA and LAU target association and activity. 


\section{Chapter 3. Assessment of the role of E127 and K124 $\beta$ I-tubulin amino acids in the activity of microtubule stabilising agents}

\subsection{Introduction}

\subsubsection{Cytotoxicity screening and MTT assays}

The continued discovery of new natural products, along with the ongoing construction of synthetic novel compound libraries, demands the ability to screen these chemicals in a highthroughput fashion to determine the existence and extent of any bioactivity. These assays can also be applied further downstream in the drug development pipeline to direct target identification and lead optimisation studies. The particular type of screening assay used depends on a specific bioactivity to be identified or any predicted action of a set of materials to be tested. For example, compounds may be screened for their cytotoxic abilities as a desirable property for further drug development, particularly in areas such as cancer. This may be done using assays that measure cytotoxicity or anti-proliferative effects, one of the most common being the MTT assay. This utilises 3-(4,5-Dimethyl-2-thiazolyl)-2,5-diphenyl2H-tetrazolium (MTT), a water-soluble salt that is reduced to an insoluble purple formazan product in proliferating cells. ${ }^{84}$ More specifically, MTT is reduced by cytosolic NADPHdependent oxidoreductase enzymes and therefore gives an indication of cellular metabolic activity as measured by the optical absorbance of the formazan product at $570 \mathrm{~nm} .{ }^{85}$ By using this robust and straightforward assay to quantitate cell metabolic activity under different conditions, such as drug treatment, the concentration-response of different compounds in specific cell lines can be determined.

\subsubsection{Cell cycle analysis}

As MTAs, PLA and LAU effect their cytotoxicity, at least in part, through the inhibition of mitotic spindle function and cell division. As a result, a cell's normal life cycle is halted causing a build-up of cells within a population in the $G_{2} / M$ phases. This characteristic cell cycle arrest has been used in numerous previous MTA studies as a biomarker of their activity. The typical protocol this is using the DNA-intercalating stain PI and measuring its fluorescence with flow cytometry. As PI binds to DNA and fluoresces at $533 \mathrm{~nm}$ this can be used to quantitate the DNA content within cells and hence which stage of the cell cycle they are in at the time of fixation. As cells in the $\mathrm{G}_{2} / \mathrm{M}$ phases have double copies of DNA 
compared to those in $\mathrm{G}_{1}$, cells in these stages will be represented by a peak in PI fluorescence twice that of $G_{1}$. Therefore, an increase in the proportion of cells in a given population contained in this peak as a result of MTA treatment will signify the associated mitotic arrest.

\subsubsection{In situ microtubule polymerisation}

As discussed previously, microtubules are highly dynamic structural polymers and as such undergo constant polymerisation and depolymerisation in response to cellular functional needs, particularly during the processes governing cell division. Upon the treatment of cells with MTAs, the equilibrium between these different tubulin states is altered. PTX, IXA, PLA and LAU, all being MSAs, act to stabilise these proteins, thereby increasing the proportion of polymerised tubulin. By exploiting the differences in solubility between polymerised (insoluble) and non-polymerised (soluble) tubulin, these fractions can be separated and quantified in order to examine such drug effects. Fractionation of tubulin protein has been used previously to study the effects of PTX on microtubule dynamicity, ${ }^{86}$ as well as PLA and LAU more recently. ${ }^{74}$ It has therefore been utilised for the purposes of this investigation to determine the effects of the mutations of interest on the stabilising activity of these compounds.

\subsection{Aims}

3.2.1 Determine the concentration-response of peloruside $A$ and laulimalide in cells with BI-tubulin containing either the K124A or E127A point mutations

The following experiments will seek to assess the role that the amino acids E127 and K124 play in the binding of PLA and LAU to their protein target. The primary evidence for this will be gathered from measuring any changes in concentration-response from cell proliferation assays involving cells containing either of the two potential H-bond forming amino acids mutated to the relatively inert alanine. It is hypothesised that these mutations will alter the response of HEK293 cells towards the marine MSAs, perhaps to varying degrees depending on the mutation and drug. These effects will be isolated by comparison to cells transfected with wild type and the previously studied D297I mutant $\beta I$-tubulin, as well as treatment with the taxane-site MSAs PTX and IXA. 


\subsubsection{Evaluate the effect of the E127A mutation on cell cycle arrest caused by peloruside A}

In order to probe any changes in drug binding caused by the studied mutations, secondary evidence will be sought through the analysis of cell cycle arrest. If a transfected cell population expressing mutant $\beta \mathrm{I}$-tubulin exhibits an increased resistance to MSA-induced cytotoxicity, it is thereby hypothesised that a similar reduction of $\mathrm{G}_{2} / \mathrm{M}$ cell cycle block would also be observed. As this has been studied for the K124 mutation previously in the Miller lab group (unpublished), this investigation will focus solely on the effects of the E127A mutation on PLA activity.

3.2.3 Evaluate the effect of the E127A mutation on in situ microtubule dynamics in response to peloruside $A$

In addition to determining any impact on cell proliferation and cell cycle arrest that these mutations may have, it is also important to understand the mechanism by which these changes occur. Due to the mechanism of action of MSAs, this can be investigated by examining the effect $\beta \mathrm{I}$-tubulin subunits containing these aberrant amino acids have on microtubule dynamic instability. For example, increased resistance to these drugs would suggest that stabilisation of its microtubules is reduced and hence less polymerised tubulin protein would be observed.

\subsubsection{Visualise the effect of the E127A mutation on the regular structure of microtubules}

Along with the quantitative measures of MSA function in E127A expressing cells, it will also be valuable to qualitatively assess the functional significance of mutant $\beta I$-tubulin subunits in dividing and pre-apoptotic cells. This will be achieved using fixed-cell confocal microscopy and immunocytochemistry. 


\subsection{Materials and Methods}

\subsubsection{Determination of cell activity in E127A and K124A mutants treated with peloruside A}

and laulimalide by MTT assay

HEK293 cells were transfected with either wild type, K124A, E127A or D297I $\beta I$-tubulin, or mock-transfected (Lipofectamine $3000^{\circledR}$ only, no DNA), in 6-well plates and grown for $48 \mathrm{~h}$. They were then harvested and seeded in 96-well plates at a density of $1 \times 10^{4}$ cells per well in $90 \mu \mathrm{L}$ DMEM. After $24 \mathrm{~h}$, each cell sub-type was treated with each of the four drugs: PTX, IXA, PLA and LAU. This was done by first creating a serial dilution panel of each compound on a 96-well plate. DMEM $(100 \mu \mathrm{L})$ was first added to each well, followed by $100 \mu \mathrm{L}$ of a 5 $\mu \mathrm{M}$ stock solution of each drug in duplicate, so that the resulting concentration in those wells was $2.5 \mu \mathrm{M}$. Half-log concentration serial dilutions were then carried out to give a total of 13 different concentrations of each compound. Two control wells with no drug and two blank wells with no cells were also included for every drug. Ten $\mu \mathrm{L}$ from each of the wells in the drug panels was then added to the corresponding wells for every transfection type, resulting in final volumes of $100 \mu \mathrm{L}$ per well and $10 \mathrm{x}$ dilutions of the original drug concentrations (i.e. beginning with $250 \mathrm{nM}$ ). Plates were incubated at $37^{\circ} \mathrm{C}$ for $72 \mathrm{~h}$, after which time $20 \mu \mathrm{L}$ of MTT (5 mg/mL in PBS) was added to every well. Plates were incubated for a further $2 \mathrm{~h}$ at $37^{\circ} \mathrm{C}$ to allow for purple formazan crystal formation. A $100 \mu \mathrm{L}$ volume of solubilising solution (10\% SDS, 45\% DMF in Milli-Q water ( $\mathrm{pH} 4.5)$ ) was then added and the plates left to incubate in the dark at $37^{\circ} \mathrm{C}$ overnight. Infrared absorbance at $570 \mathrm{~nm}$ was measured using an Enspire ${ }^{\circledR} 2300$ spetrophotometric plate reader (Perkin Elmer, Waltham, MA, USA). Absorbance values for the blank wells in each plate were used as a background level, while values for treated wells were normalised against those for untreated controls. In this way, the viability of treated cells was represented by a percentage of control adjusted for background absorbance. These data were then used to plot cell viability curves, utilising non-linear regression models to determine the half-maximal inhibitory concentration values $\left(\mathrm{IC}_{50}\right)$ as a measure of drug activity for each MSA in each transfection variant. This modelling, as well as subsequent statistical analyses, was done using Prism 7 software (GraphPad, San Diego, CA, USA). Four biological replicates were carried out to ensure accuracy of results and improve the power of statistical comparisons. 


\subsubsection{Cell cycle analysis of E127A mutants in response to peloruside A by flow cytometry}

HEK293 cells were seeded in 6-well plates at a density of $5 \times 10^{5}$ cells per well and transfected with either wild type or E127A mutant $\beta$ I-tubulin. After $48 \mathrm{~h}$, growth medium in each well was replaced with DMEM containing IXA or PLA at concentrations of 0,40 or 100 $\mathrm{nM}$. After a further $16 \mathrm{~h}$ incubation, cells were harvested with $\operatorname{TrypLE}^{\mathrm{TM}}$ and centrifuged at $300 \mathrm{~g}$ for $4 \mathrm{~min}$. From each well, $1 \times 10^{6}$ cells were transferred to round-bottom polystyrene tubes (Fisher Scientific) and washed with $1 \mathrm{~mL}$ cold PBS. After centrifugation, the cell pellets were re-suspended in $100 \mu \mathrm{L}$ PBS and fixed by adding $1 \mathrm{~mL}$ of zinc-based fixative (0.1 M Tris- $\mathrm{HCl}$ ( $\mathrm{pH} 7.8), 0.05 \%$ calcium acetate, $0.5 \%$ zinc chloride, $0.5 \%$ zinc acetate) to each tube while vortexing. The samples were stored at $4^{\circ} \mathrm{C}$ overnight, after which time they were centrifuged at $600 \mathrm{~g}$ for $5 \mathrm{~min}$. They were then washed twice with $1 \mathrm{~mL}$ of FACS buffer by centrifugation at $300 \mathrm{~g}$ for $4 \mathrm{~min}$, and stained for DNA content by resuspension in $500 \mu \mathrm{L}$ Kirshan buffer $(0.1 \%$ sodium citrate, $0.05 \mathrm{mg} / \mathrm{mL}$ PI, $0.2 \%$ NP-40, $0.05 \mathrm{mg} / \mathrm{mL}$ RNase A, 1 drop/100 mL $0.1 \mathrm{~N} \mathrm{HCl}$ in Milli-Q water). In order to accurately determine the concentration of double stranded DNA in the cells, RNase is needed to break down any double stranded RNA present. PI then intercalates with and stains DNA only. Samples were incubated at $4{ }^{\circ} \mathrm{C}$ for $1 \mathrm{~h}$ before being analysed by flow cytometry with a BD FACSCanto II flow cytometer (BD Biosciences). At least 10,000 events were recorded for each sample, and cells were sorted based on GFP fluorescence. GFP and PI negative controls were used for compensation due to the overlapping emission spectra for the two fluorophores. GFP positive cells were analysed with the PI filter and grouped into $G_{1}, S$ and $G_{2} / M$ phases of the cell cycle based on DNA content using FlowJo software (FlowJo).

\subsubsection{In situ polymerisation of E127A mutants in response to peloruside $A$ by western} blotting

HEK293 cells were seeded in 6-well plates at a density of $5 \times 10^{5}$ cells per well and transfected with either wild type or E127A mutant $\beta$ I-tubulin. After $48 \mathrm{~h}$, growth medium in each well was replaced with DMEM containing IXA or PLA at concentrations of 0,40 or 100 $\mathrm{nM}$. After a further $16 \mathrm{~h}$ incubation, cells were rinsed twice with PBS prior to lysis with 400 $\mu \mathrm{L}$ hypotonic buffer ( $1 \mathrm{mM} \mathrm{MgCl} 2,2 \mathrm{mM}$ EGTA, $0.15 \%$ Tergitol, $50 \mathrm{nM}$ Tris- $\mathrm{HCl}$ (pH 6.8)) supplemented with $10 \mu \mathrm{L} / \mathrm{mL}$ protease inhibitor cocktail I (Sigma-Aldrich) immediately prior to use. Cells were incubated in the dark at $37^{\circ} \mathrm{C}$ for $5 \mathrm{~min}$, after which time cells were 
removed from the wells by cell scraping and the resulting solution was transferred to $1.5 \mathrm{~mL}$ Eppendorf tubes. Soluble and insoluble tubulin fractions in each sample were then separated by centrifugation at $14,000 \mathrm{~g}$ for $10 \mathrm{~min}$ at RT. The supernatant containing the soluble protein was transferred to new $1.5 \mathrm{~mL}$ Eppendorf tubes, and the remaining cell pellet of insoluble material was resuspended in a further $400 \mu \mathrm{L}$ of lysis buffer. All samples were kept on ice from this point onwards. Each sample was prepared for electrophoretic separation by diluting $24 \mu \mathrm{L}$ of protein-containing solution in $6 \mu \mathrm{L}$ of $5 \mathrm{x}$ Laemmli reducing buffer with $10 \% \beta$-mercaptoethanol and boiling at $95^{\circ} \mathrm{C}$ for $5 \mathrm{~min}$ to completely denature proteins. The reminder of the protocol follows the same procedure as described above in section 2.3.8. However, the anti- $\beta$-actin primary antibody is omitted from the immunological probing step.

\subsubsection{Assessment of E127A mutant microtubule stabilisation in response to peloruside $A$ by} immunocytochemistry and fixed cell confocal microscopy

HEK293 cells seeded onto Histogrip ${ }^{\circledR}$-treated coverslips in 6-well plates were transfected with either wild type or E127A mutant $\beta$ I-tubulin. After $48 \mathrm{~h}$ transfection medium was replaced by fresh growth medium supplemented with 0 or 100 nM IXA or PLA. From this point coverslips were processed and imaged in the same way as described in section 2.3.9.

\subsection{Results}

\subsubsection{Response of transfected HEK293 cells to microtubule stabilising agents}

The response of HEK293 cells transfected with wild type, K124A, E127A or D297I BItubulin, or those mock transfected, to PTX, IXA, PLA and LAU were tested using the MTT assay described in section 3.3.1. PTX and IXA were used as negative controls, as these are known to act through the taxane binding site on $\beta$-tubulin, which is a large molecular distance away from the location of the mutated amino acids. By measuring the $\mathrm{IC}_{50}$ of each of these drugs, any specific impact the mutations have on PLA or LAU activity can be isolated. As the E127A and K124A point mutations should not affect binding of compounds to the taxane region it is no changes in response to PTX and IXA are expected in the mutant cells compared to wild-type. Unfortunately, PTX was unable to be used to produce meaningful results due to the consistent generation of near-linear concentration-response curves rather than the typical sigmoidal models expected for a drug interaction with its target protein (Figure 3.4.1). While the reason for this is unclear, it has been suggested that PTX adhering 
to plastic pipette tips may result in improper serial dilutions. IXA, on the other hand, produced useable curves from four parameter non-linear regression analysis, with all $\mathrm{R}^{2}$ values greater than 0.9 (Figure 3.4.2). From these results, no significant differences in $\mathrm{IC}_{50}$ were observed between wild type and each of the mutations with IXA treatment, including the positive control mutation D297I ( $\mathrm{P}>0.05$, Figure 3.4.3).

$$
\text { Paclitaxel }
$$

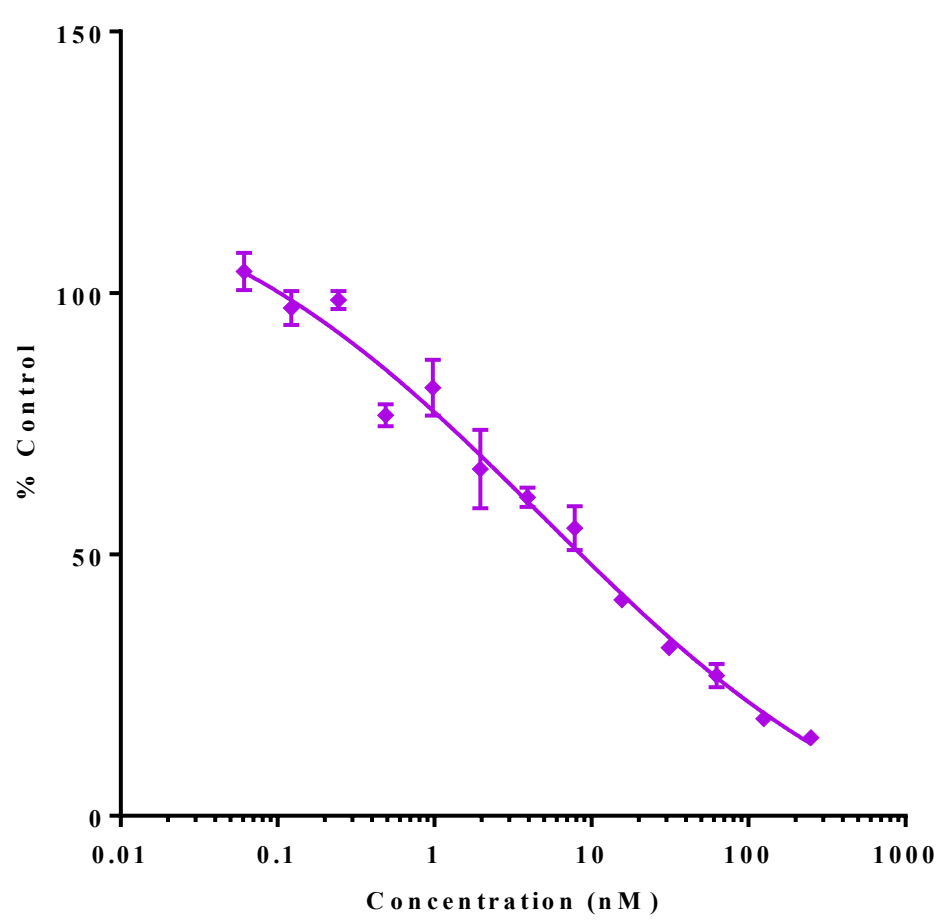

Figure 3.4.1. Representative concentration-response curve for cells treated with PTX. Graph shows mock transfected cell activity as determined by the MTT assay normalised against the untreated control (\%) vs. PTX concentration (log scale). Each point represents the mean response from two technical repeats at each concentration ranging from $250 \mathrm{nM}$ to 0.06 $\mathrm{nM}$. The line shows the fit of a four-parametric non-linear regression model used to determine $\mathrm{IC}_{50}$ values from Prism analysis software. Error bars represent SEM. 

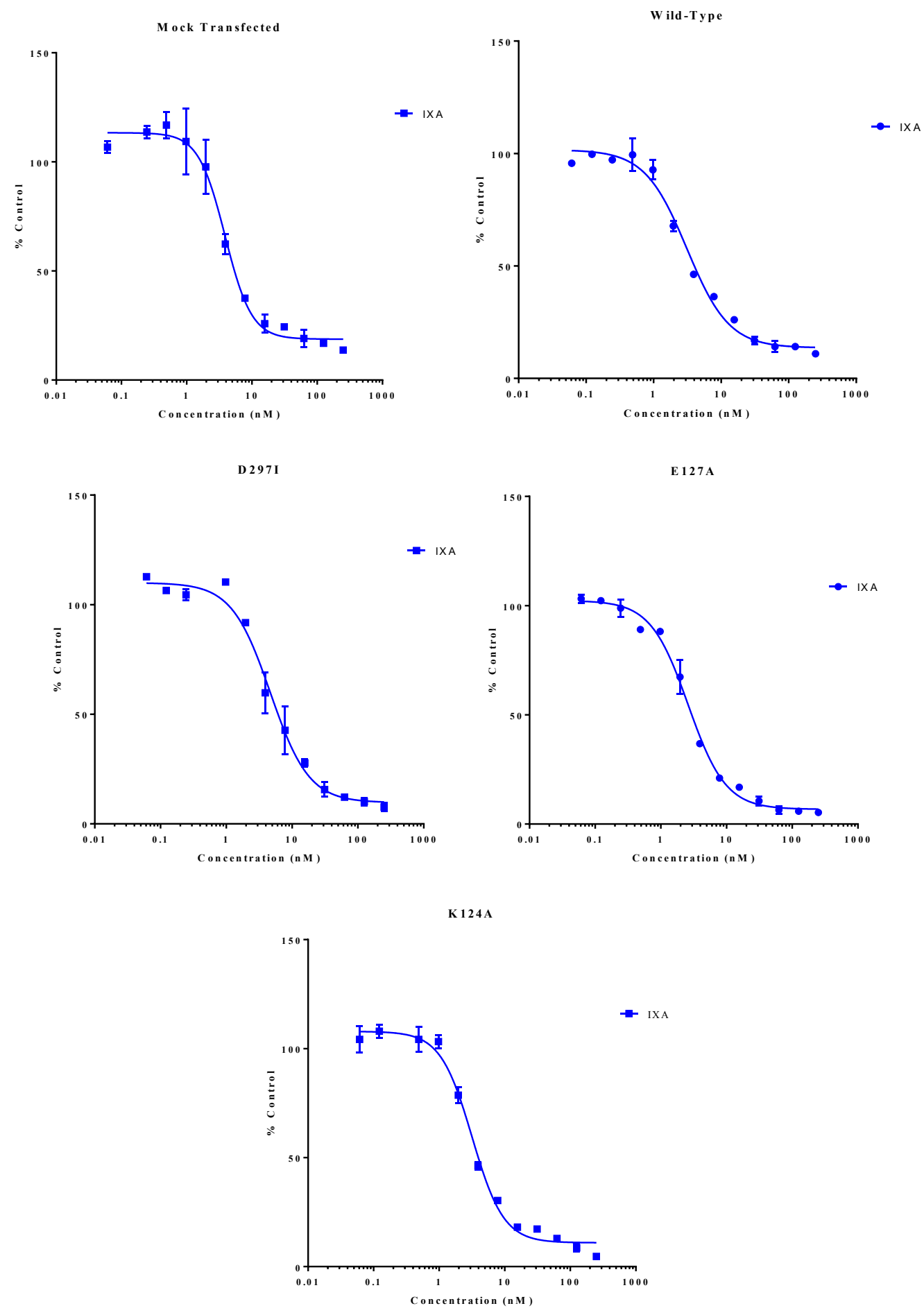

Figure 3.4.2. Representative concentration-response curves for cells treated with IXA. Graphs show cell activity as determined by the MTT assay normalised against the untreated control (\%) vs. IXA concentration (log scale). Each point represents the mean response from two technical repeats at each concentration ranging from $250 \mathrm{nM}$ to $0.06 \mathrm{nM}$. The line shows the fit of a four-parametric non-linear regression model used to determine $\mathrm{IC}_{50}$ values from Prism analysis software. Error bars represent SEM. 


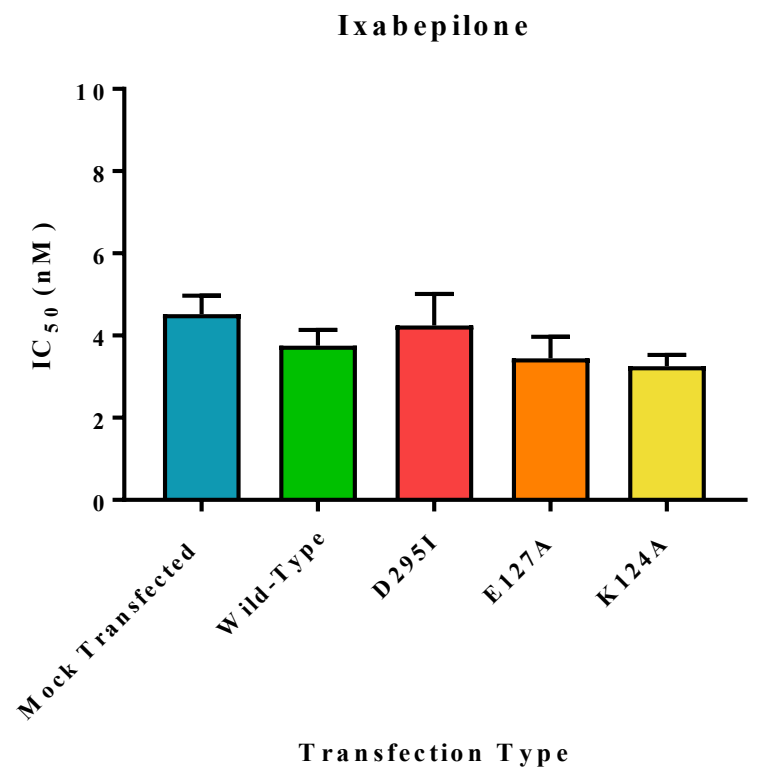

Figure 3.4.3. Summary $I_{C_{50}}$ results for MTT assays with IXA. Bar graph showing mean IXA $\mathrm{IC}_{50}$ values for each transfection category determined by repeated MTT assays. Error bars represent SEM from four biological repeats. None of the results were statistically different from the wild-type transfected control ( $\mathrm{P}>0.05$ by Student's t-test).

Based on the lack of change in sensitivity to IXA between the wild-type and mutant transfected cells, any alteration in $\mathrm{IC}_{50}$ observed for PLA and LAU can likely be attributed to drug-tubulin binding effects. Concentration-response curves demonstrating this analysis of PLA and LAU for each transfection category are shown in Figure 3.4.4. No changes in $\mathrm{IC}_{50}$ were seen for the studied mutations, with no significant differences between wild-type and E127A or K124A transfected cells for either compound ( $\mathrm{P}>0.05$, Student's t-test, Figure 3.4.5). It is important to note that while both drugs gave high quality dose-response curves with $\mathrm{R}^{2}$ values above 0.9 , the $\mathrm{IC}_{50}$ values generated for LAU were at least 10 -fold higher than seen in previous experiments, suggesting likely degradation of the compound in the stock supply (figure 3.4.4). For this reason, the subsequent assays focussed solely on PLA. In contrast to the lack of change in response to PLA or LAU with the E127A or K124A mutations, a significant increase in the $\mathrm{IC}_{50}$ of PLA was found between wild-type and the positive control mutation D297I ( $\mathrm{P}<0.01$, Student's t-test). This corresponds to a $60 \pm 10 \%$ increase in resistance to PLA, a similar effect to results reported in a previous study. Along with the lack of change in $\mathrm{IC}_{50}$ for IXA, this helps to validate the evidence gathered in the present study. It affords inferential power to any conclusions drawn from the results 
regarding the role of E127 and K124 in the binding of PLA specifically to $\beta$-tubulin. Finally, no significant difference was observed between mock-transfected and wild-type for IXA and PLA ( $\mathrm{P}>0.05$, Student's t-test), suggesting that transfection does not alter the proliferation of cells. However, a significantly difference was seen for the LAU $(\mathrm{P}<0.01$, Student's t-test $)$, which may be due to inconsistencies arising from this compromised compound. 

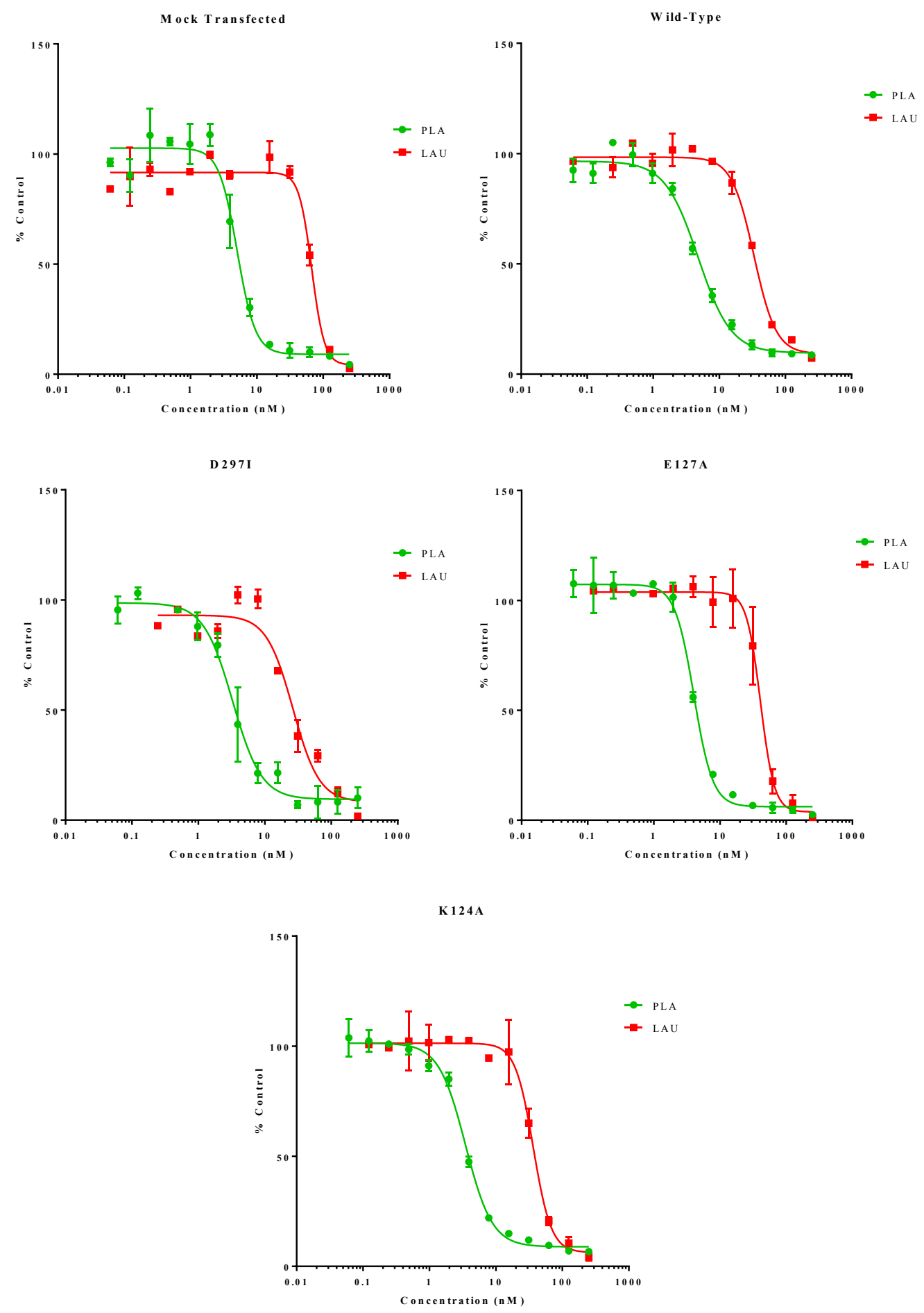

Figure 3.4.4 Representative concentration-response curves for cells treated with PLA and LAU. Graphs show cell proliferation as determined by the MTT assay normalised against the untreated control (\%) vs. PTX and LAU concentration (log scale). Each point represents the mean response from 2 technical repeats at each concentration ranging from 250 $\mathrm{nM}$ to $0.06 \mathrm{nM}$. The line shows the fit of a four-parametric non-linear regression model used to determine $\mathrm{IC}_{50}$ values from Prism analysis software. Error bars represent SEM. 
Peloruside A
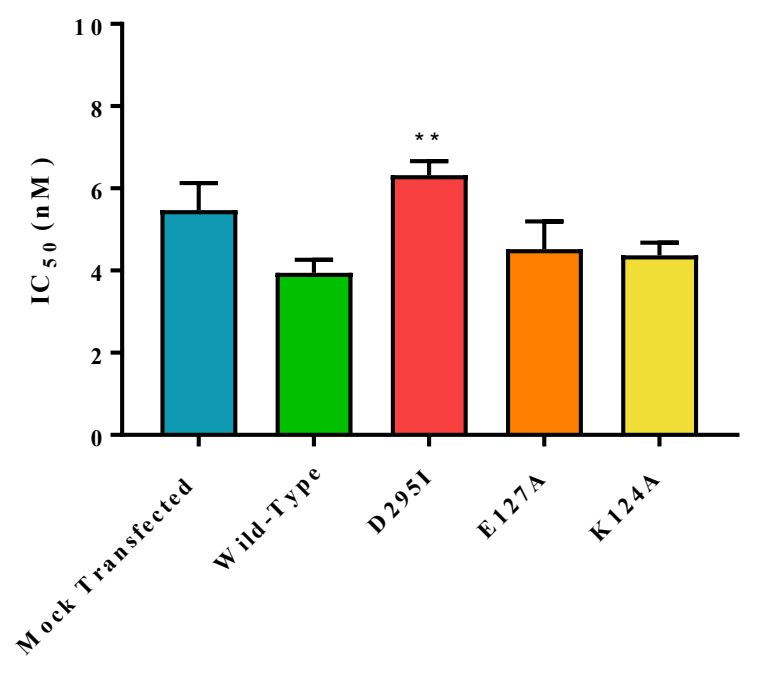

Transfection Type
L a u lim alid e

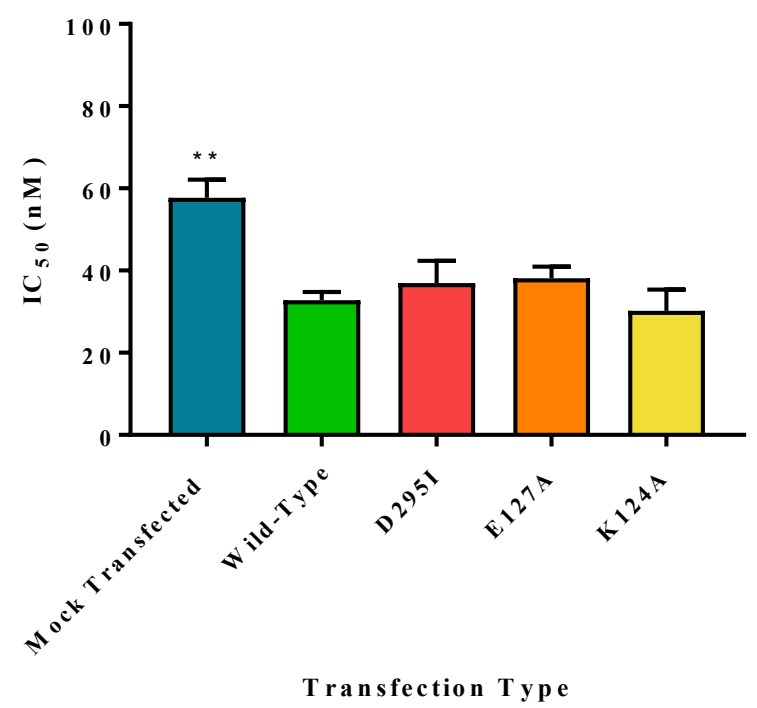

Figure 3.4.5. Summary $I_{50}$ results for MTT assays with PLA and LAU. Bar graph showing mean PLA and $\mathrm{LAU} \mathrm{IC}_{50}$ values for each transfection category determined by repeated MTT assays. Error bars represent SEM from four biological repeats. $* *=\mathrm{P}<0.01 \mathrm{vs.}$ wild-type by Student's t-test.

\begin{tabular}{|c|c|c|c|c|c|}
\hline & Mock Control & Wild-Type & D297I & $E 127 A$ & $K 124 A$ \\
\hline$I X A$ & $4.5 \pm 0.4$ & $3.8 \pm 0.4$ & $4.2 \pm 0.4$ & $3.4 \pm 0.5$ & $3.3 \pm 0.3$ \\
\hline$P L A$ & $5.5 \pm 0.7$ & $3.9 \pm 0.3$ & $6.3 \pm 0.3 * *$ & $4.5 \pm 0.7$ & $4.4 \pm 0.3$ \\
\hline$L A U$ & $57.7 \pm 4.4^{* *}$ & $32.8 \pm 2.0$ & $36.9 \pm 5.4$ & $38.1 \pm 2.8$ & $30.2 \pm 5.1$ \\
\hline
\end{tabular}

Table 3.4.1. Summary of $\mathrm{IC}_{50}$ results for MTT assays. Table shows the mean $\pm \mathrm{SEM} \mathrm{IC}_{50}$ values $(\mathrm{nM})$ for each drug and each transfection type from four biological repeats. $* *=$ $\mathrm{P}<0.01$ vs. wild-type by Student's t-test.

\subsubsection{Analysis of E127A transfected HEK293 cell cycle arrest caused by peloruside A}

In order to determine if any changes to the $\mathrm{G}_{2} / \mathrm{M}$ phase cell cycle block caused by PLA result from the introduction of the E127A mutation into $\beta$ I-tubulin, flow cytometry with PI nucleic acid staining was employed. This mitotic arrest is characteristic of MSA bioactivity. It presents as a concentration-dependent increase in the proportion of cells in the $\mathrm{G}_{2} / \mathrm{M}$ phase of 
the cell cycle with drug treatment. Therefore, if the glutamate 127 residue is important for PLA binding to $\beta$-tubulin and subsequent microtubule stabilisation, it is expected that this increase would be reduced.

The proportion of cells in each phase of the cell cycle is determined based on the fluorescence of PI, which intercalates with double stranded DNA. The resulting histogram gives two distinct peaks. The first corresponds to cells with two copies of genomic DNA, one in each chromosome of the sister chromatids $\left(\mathrm{G}_{1}\right.$ phase, $\left.2 \mathrm{n}\right)$, and the second to cells with double the amount of DNA prior to and during mitosis $\left(\mathrm{G}_{2} / \mathrm{M}\right.$ phase, $\left.4 \mathrm{n}\right)$ The area in between represents cells undergoing DNA replication during $S$ phase $(<2 n,<4 n)$. This experiment utilised GFP fluorescence to differentiate between transfected and non-transfected cells. As described in section 2.4.1, compensation controls were used to correct for the overlap in emission spectra between GFP and PI.

A further issue arising from the use of GFP as a selection marker is the loss of fluorescent signal when fixing cells with ethanol before PI staining. This is a commonly used fixative with the advantage of also permeabilising cells, providing intracellular access to the otherwise non-membrane permeable PI. However, when applied here substantial nearcomplete loss of GFP fluorescence was experienced. Therefore, alternative fixation methods were trialled. Firstly, a live-cell DNA stain, Hoechst 33342 was used, which did not generate a signal when excited with the $405 \mathrm{~nm}$ laser and DAPI filter set. Despite the fluorescence of Hoechst 33342 under the same conditions when used for microscopy, it appears to require an ultra-violet laser for use in flow cytometry. Next, PI staining with $4 \%$ paraformaldehyde fixation was tested. While this method successfully retained GFP signal, the resulting cell cycle histograms were of poor resolution, as indicated by high coefficients of variance. Finally, a zinc-based fixative protocol was found to both successfully maintain GFP fluorescence and provide distinct PI histogram peaks for accurate cell cycle identification. This was therefore used in all the following cell cycle experiments.

Cells were analysed by flow cytometry by first gating the appropriate populations, as illustrated in Figure 3.4.6. This was done by first gating for single cells based on a forward scatter vs. side scatter plot, followed by a PI-area vs. PI-width plot of the gated population. The resulting cells were singlets, discriminating against doublets and debris. A GFP-area histogram was then used to separate the single cells into GFP positive and negative. Finally, 
PI-area histograms for each of these sub-populations were used to determine the proportion of cells in the different cell cycle phases.

A
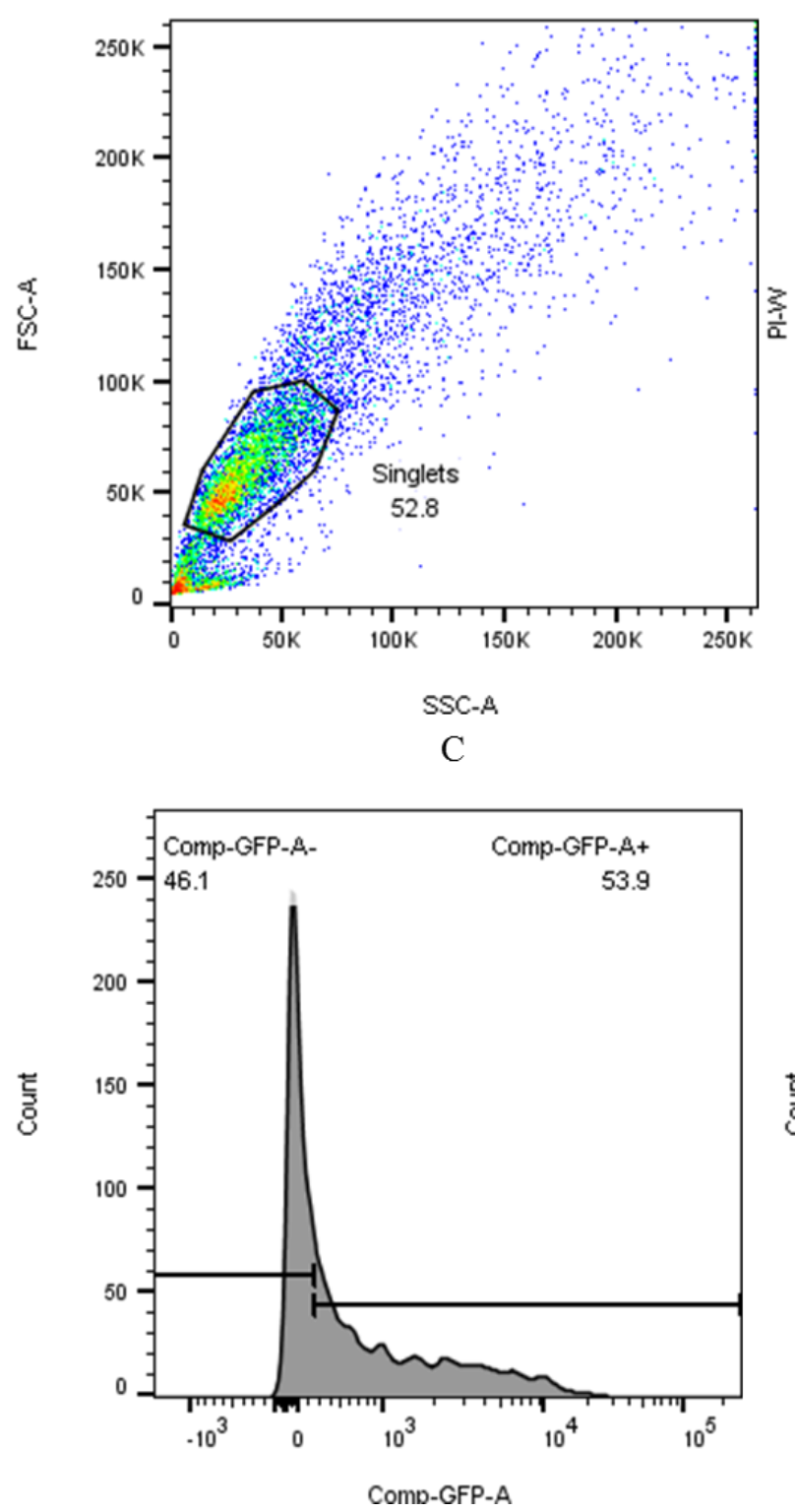

B

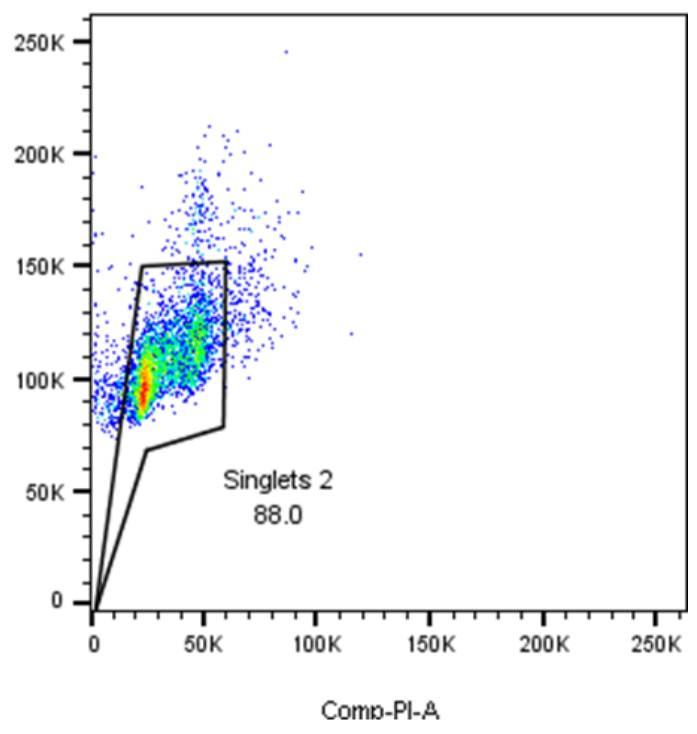

$\mathrm{D}$

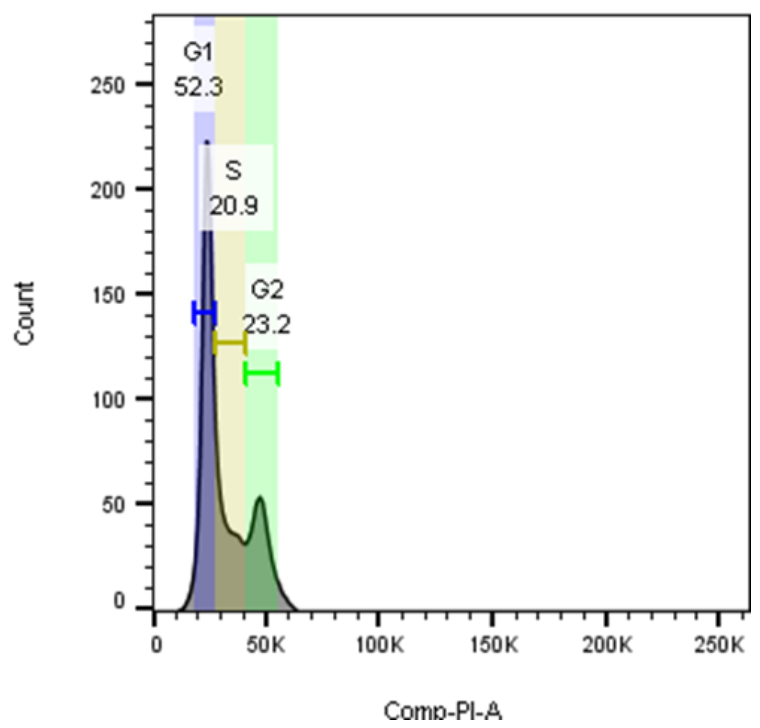

Figure 3.4.6. Flow cytometry workflow cell cycle analysis. This figure shows flow cytometry results from one transfected cell sample to demonstrate typical analytical workflow. (A) Forward scatter (y-axis) vs. side scatter (x-axis) of total events, gating for singlets. (B) PI-width (y-axis) vs. PI-area (x-axis) to further select for non-apoptotic single cells. (C) Gated events displayed on GFP-area histogram, gating for GFP- and GFP+ cells. (D) Each population plotted with PI-area histogram (linear scale) and subject to cell cycle analysis module in FlowJo software to determine the percentage of cells in each phase (purple $=\mathrm{G}_{1}$, yellow $=\mathrm{S}$, green $=\mathrm{G}_{2} / \mathrm{M}$ ). 
Figure 3.4.7 shows the PI-A histograms from a single representative biological repeat and demonstrates the general trend seen throughout the experiment. That is, for each compound no significant difference was seen in cell cycle between wild-type and E127A transfected untreated control cells, nor between GFP positive and negative populations within each sample ( $\mathrm{P}>0.05$, Student's t-test, Figure 3.4.8). However, this changed when cells were treated with increasing drug concentrations. GFP positive wild-type cells treated with IXA exhibited an increase in the mean proportion of cells in the $\mathrm{G}_{2} / \mathrm{M}$ phase from $22 \pm 1 \%(0 \mathrm{nM})$ to $38 \pm 3 \%(40 \mathrm{nM})$ and $45 \pm 4 \%(100 \mathrm{nM})$. On the other hand, there was a significant decrease in this proportion for GFP positive E127A transfected cells, treated with both $40 \mathrm{nM}$ and $100 \mathrm{nM}$ IXA $(\mathrm{P}<0.05$, Student's t-test $)$. However, this effect is not seen for GFP negative cells, with no significant difference between wild-type and E127A for either $40 \mathrm{nM}$ or 100 nM IXA ( $\mathrm{P}>0.05$, Student's t-test). Similar results were seen for PLA. The mean percentage of wild-type GFP positive cells in $\mathrm{G}_{2} / \mathrm{M}$ phase increased form $22 \pm 1 \%$ for untreated control to $31 \pm 2 \%$ and $36 \pm 1 \%$ when treated with $40 \mathrm{nM}$ and $100 \mathrm{nM}$ PLA, respectively (Figure 3.4.8). Whereas, GFP positive E127A transfected cells treated showed significantly lower $\mathrm{G}_{2} / \mathrm{M}$ events for both $40 \mathrm{nM}$ and $100 \mathrm{nM}$ compared to wild-type $(\mathrm{P}<0.05$, Student's t-test). Again, this was in contrast to the GFP negative population, which showed no significant difference between transfection types for any concentration of PLA ( $\mathrm{P}>0.05$, Student's t-test). 

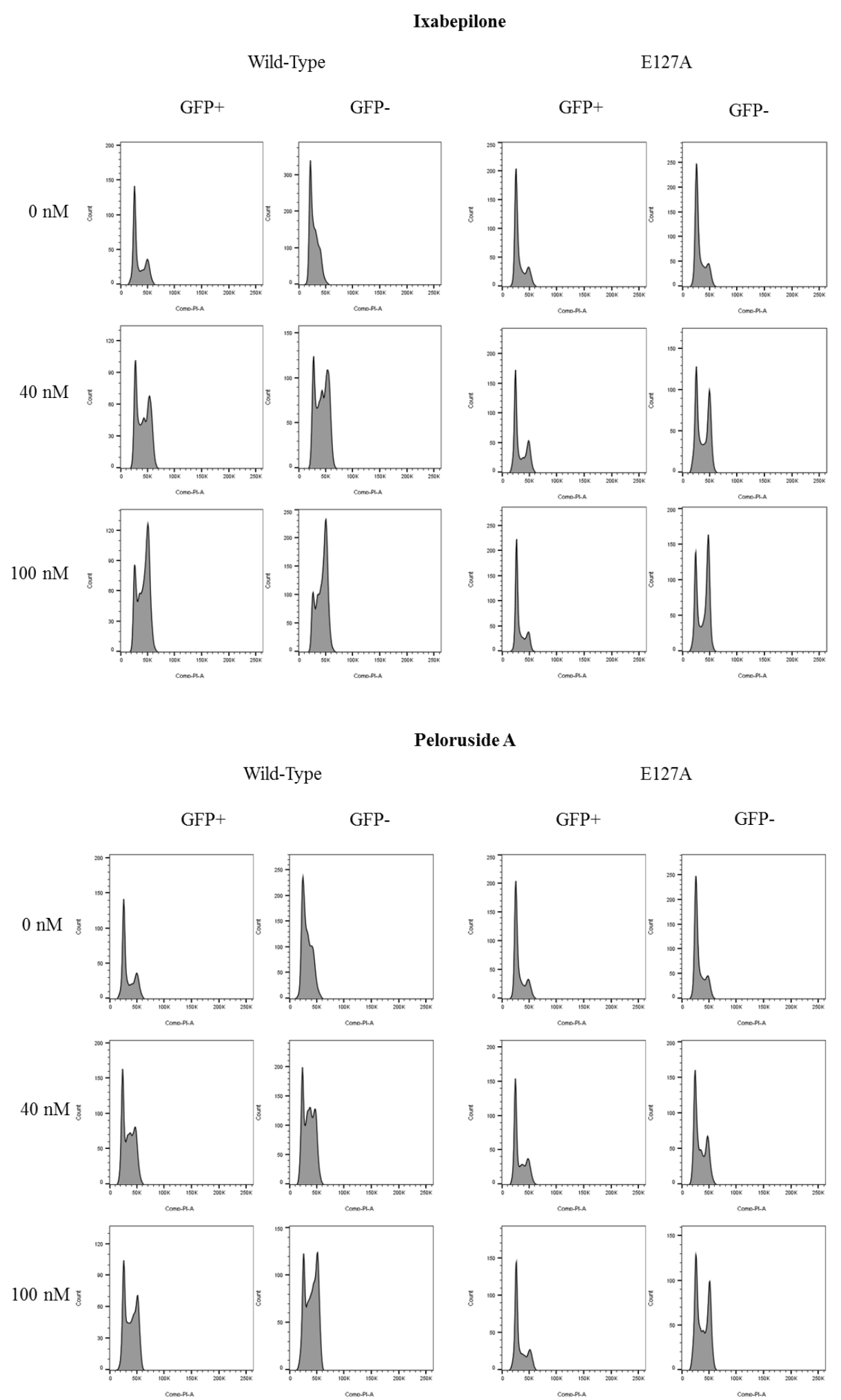

Figure 3.4.7. Cell cycle analysis for cells treated with IXA and PLA. Histograms show cell cycle results for one biological repeat from the cell cycle assay. They display distinct features corresponding to different cell cycle phases (see figure 3.4.6). Examples given for both wild-type and E127A mutant $\beta$ I-tubulin transfected cells, treated with three different concentrations of IXA and PLA, separated by gating into GFP- and GFP+ populations. 

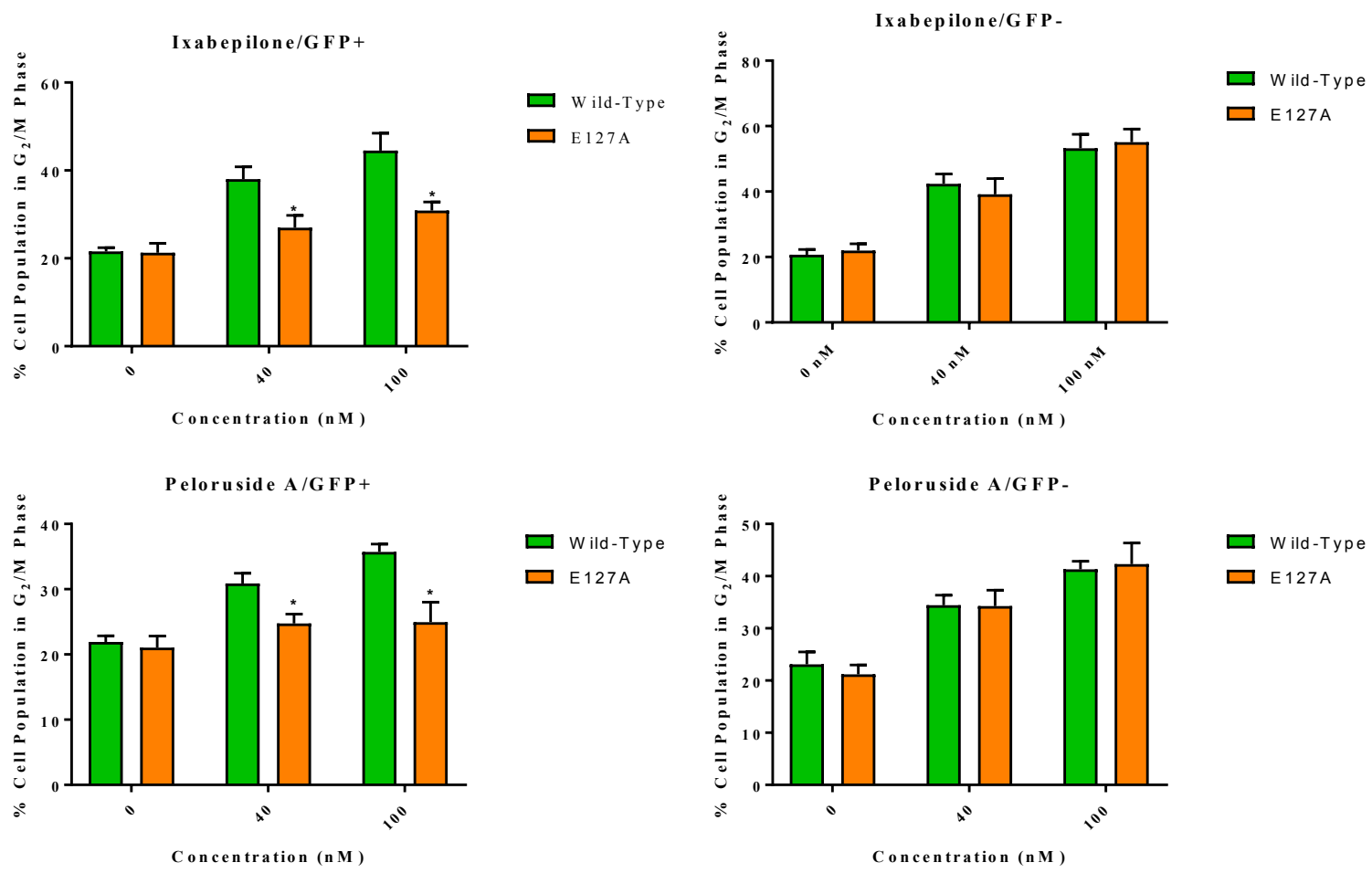

Figure 3.4.8. $\mathbf{G}_{2} / \mathrm{M}$ block caused by IXA and PLA. Bar graphs show combined results from cell cycle experiments. Results shown for both wild-type and E127A mutant $\beta I$-tubulin transfected cells, treated with three different concentrations of IXA and PLA, divided into GFP+ and GFP- populations. Graphs show mean percentage of the total cell population in the $\mathrm{G}_{2} / \mathrm{M}$ phase for each concentration. Error bars represent SEM from three biological repeats. $*=\mathrm{P}<0.05$ vs. wild-type for a particular concentration by Student's t-test.

\subsubsection{In situ polymerisation of E127A mutant BI-tubulin in response to peloruside $A$}

The dynamic nature of microtubule polymers is an essential characteristic to their function as the major structural and transport machinery within cells. As microtubules cycle between growth and catastrophe the subunits undergo polymerisation and depolymerisation, changing between insoluble and soluble states, respectively. As these processes are disrupted by the stabilising effects of MSAs, treatment with PLA can lead to changes dynamic equilibrium and promote the polymerisation of tubulin heterodimers. A protocol utilising western blotting to asses this in situ polymerisation has been used in a number of previous studies to determine the effects of MTAs. ${ }^{73,86}$ Unfortunately, when employed in the current project to further probe the impact of the E127A mutation on PLA activity very few meaningful blots were produced. Similar to the results seen in the transfection efficiency western blotting 
procedure described in section 2.4.6, little to no $\beta$-tubulin was observed on membranes from six biological repeats. Again, after troubleshooting it issue appears to stem from a high degree of sample degradation or unsuitable transfer conditions, or both. However, one blot did show faint bands at $75 \mathrm{kDa}$ corresponding GFP-tagged $\beta$-tubulin for wild-type transfected cells treated with 0, 40 and $100 \mathrm{nM}$ PLA (Figure 3.4.9). Analysis of band intensities showed that, as expected, the proportion of $\beta$-tubulin in the polymerised fraction increased when treated with $100 \mathrm{nM}$ PLA, supporting to the compound's stabilising effect on microtubules. While this single result does not contribute any new evidence to help determine the role of E127 in PLA binding, it is consistent with the previously reported MTT and cell cycle assays that observed normal PLA activity among wild-type $\beta I$-tubulin transfected cells. However, this result also requires validation from repeat measures.

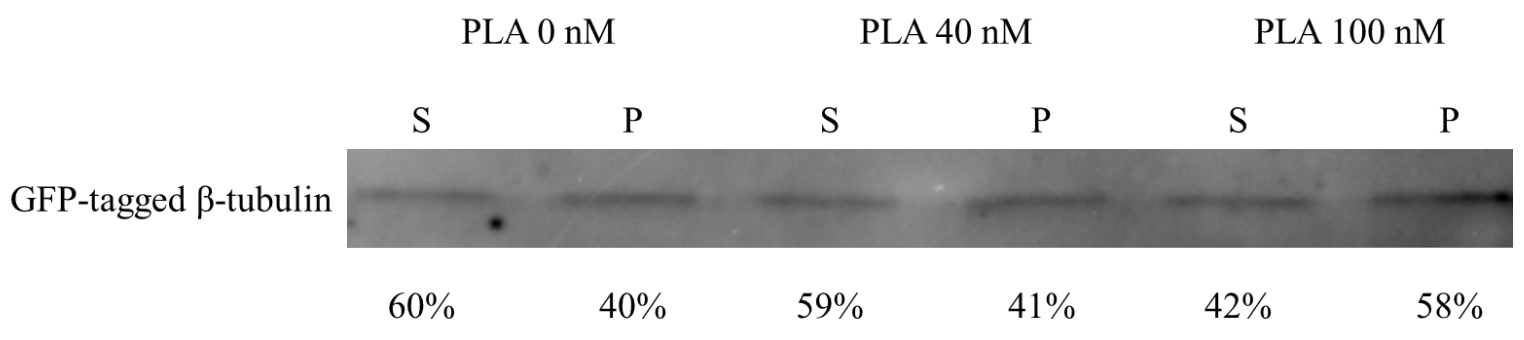

Figure 3.4.9. GFP-tagged $\beta$-tubulin bands on single in situ polymerisation western blot. Image shows bands seen at $75 \mathrm{kDa}$ for a single experiment using wild-type transfected cells treated with 0, 40 and $100 \mathrm{nM}$ PLA. Lanes are labelled based on tubulin fraction type i.e. $\mathrm{S}=$ soluble, $\mathrm{P}=$ polymerised. Values beneath bands represent percentage of combined intensity for both bands at each concentration.

3.4.4 Examination of microtubule function in response to peloruside $A$ by immunocytochemistry and confocal microscopy

While it was established in section 2.4.7 that exogenous GFP-tagged $\beta I$-tubulin subunits are successfully integrated into structurally sound microtubules, it is also valuable to assess the functional performance of these polymers when treated with MSAs. To this end, cells transfected with wither wild-type or E127A mutant $\beta$ I-tubulin were treated with $100 \mathrm{nM}$ of IXA or PLA and qualitatively assessed by immunocytochemistry and confocal microscopy (see section 3.3.4). Fixed cell samples labelled for $\beta$-tubulin and with the nucleic acid stain DAPI were assessed for the appearance of multiple asters with drug treatment among GFP- 
expressing cells, signalling the stabilisation of microtubules and the halting of cell division, leading to apoptosis (Figure 3.4.10). For wild-type cells, untreated control showed that a small proportion of GFP positive were undergoing mitosis, and these showed regular spindle formation. When treated with $100 \mathrm{nM}$ IXA and PLA, the number of dividing cells increased and many of these showed clear signs of microtubule stabilisation as multiple dense areas of GFP signal can be observed together with condensed DAPI-labelled chromatin. This effect is also consistent with GFP negative cells stained against $\beta$-tubulin, for both control and drug treated samples. On the other hand, cells transfected with E127A mutant $\beta I$-tubulin did not show the same response. In the samples imaged, GFP-expressing cells treated with $100 \mathrm{nM}$ of either compound or untreated showed signs of cell division. This is in contrast to GFPnegative cells in the same populations that showed similar morphological features as the comparable wild-type samples. 


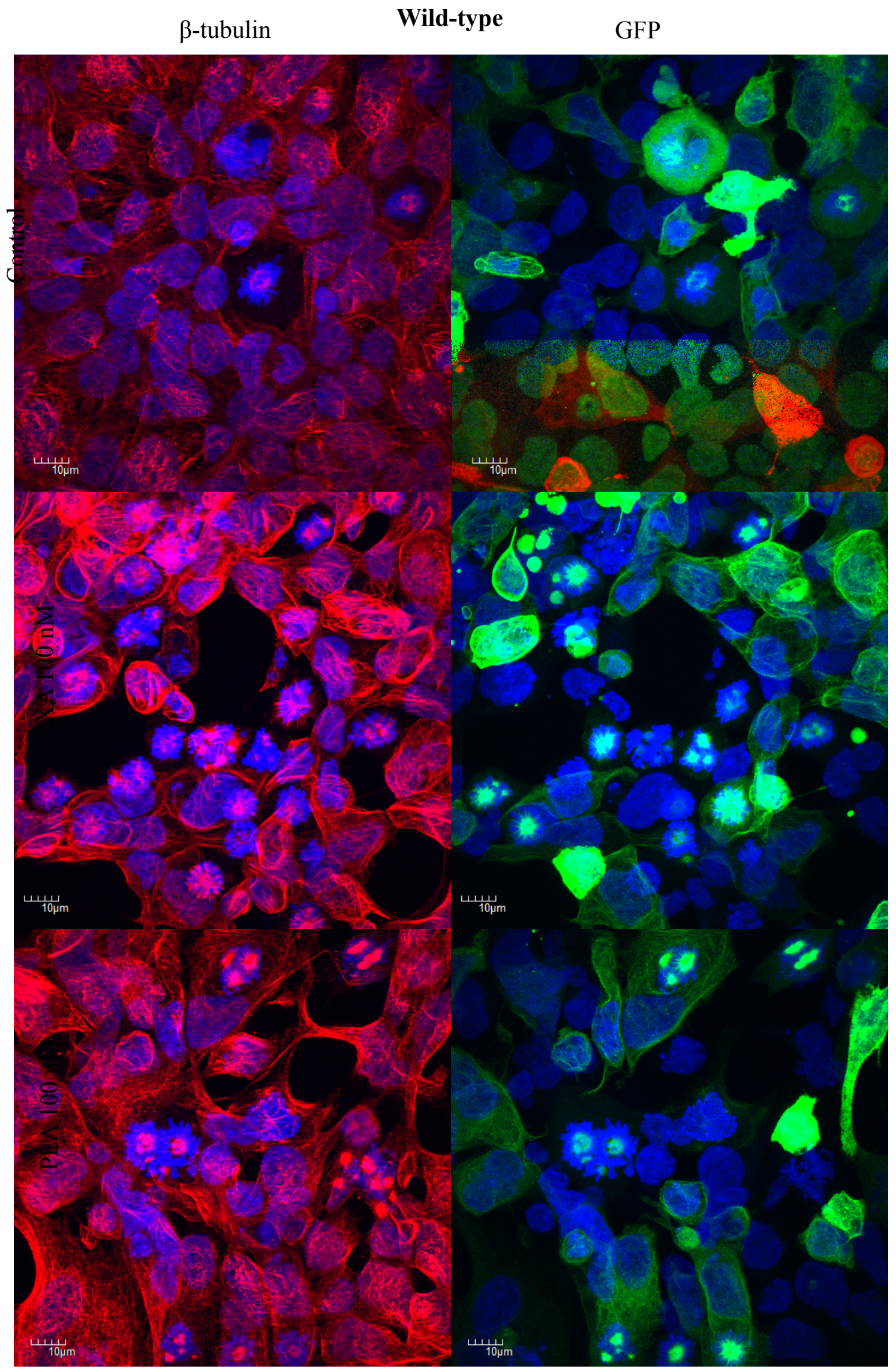




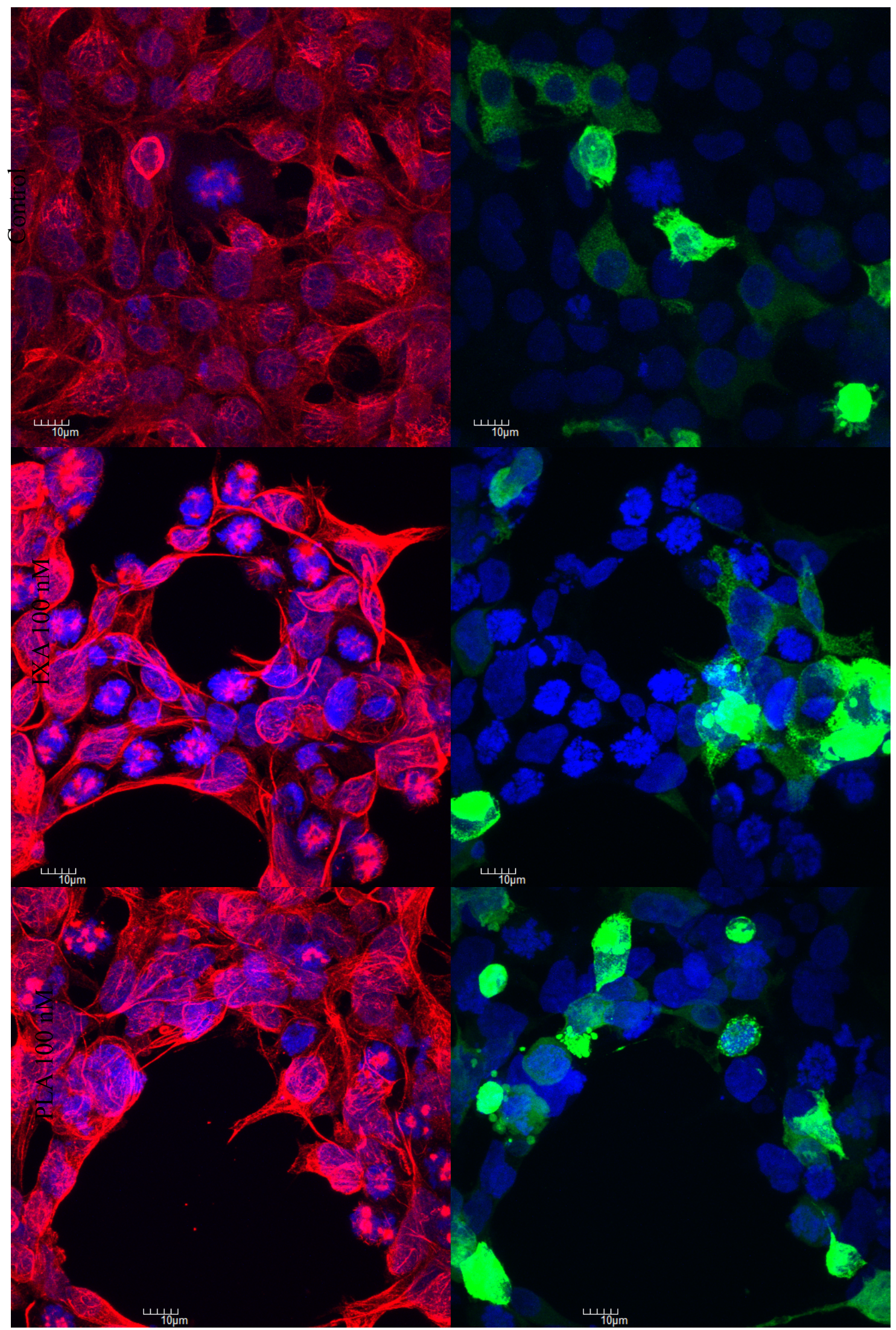


Figure 3.4.10. Confocal microscopy images of transfected HEK293 cells stained for $\beta$ tubulin. Z-stacked images show representative cells expressing wild-type and E127A mutant GFP-tagged $\beta$-tubulin. Cells are fixed onto glass coverslips pre-treated with Histogrip ${ }^{\circledR}$ and stained with Cy3-labelled antibody against $\beta$-tubulin (red signal) and the DNA stain DAPI (blue signal). Side-by-side images show Cy3 signal and DAPI signal on the left, and GFP and DAPI signal on the right. Images captured using the $100 \mathrm{x}$ oil immersion lens. For both transfection types control cells show an increase in the formation of multiple asters (areas of condensed microtubules) with increased PLA concentration, signs of microtubule stabilisation and mitotic arrest. However, where this is observed for wild-type GFP-positive tubulin expressing cells, green fluorescing microtubules do no exhibit this behaviour in E127A transfected cells.

\subsection{Discussion}

\subsubsection{The effect of $\beta$ I-tubulin mutations on cell activity in response to microtubule} stabilising agents

The purpose of this chapter is to explore the impact that the E127A and K124A point mutations have on the binding of PLA and LAU to their target region on $\beta$-tubulin. In order to isolate any such effect, it is important to first establish that these mutations do not affect the proliferation of HEK293 cells treated with MSAs that bind the taxane site. The incorporation of mutant or GFP-tagged subunits into the microtubules of cells may disrupt vital associations within the polymer structure and could therefore also alter their response to PTX or IXA. MTT assays carried out as part of this study did not show any significant differences in the proliferative performance of wild-type, mutant or mock transfected cells treated with IXA. As both glutamate 127 and lysine 124 are located far from the wellcharacterised taxane binding region through which PTX and IXA both act, this result was expected. Unfortunately, PTX did not provide any meaningful data. It instead produced unorthodox linear relationships between response and $\log$ concentration from which $\mathrm{IC}_{50}$ values with acceptable degrees of error could not be calculated. However, as both compounds bind to the same region and have similar in vitro potencies, the results for IXA were sufficient for this and subsequent experiments. Furthermore, the $\mathrm{IC}_{50}$ values determined for IXA by the MTT assays here are consistent with previous studies using mock-transfected cancer cell lines, ${ }^{86,87}$ as well as transfected HEK293 cells. ${ }^{74}$ 
The characterisation of the shared PLA/LAU binding site as distinct from the taxane region has been the product of a number of different studies. Initially, it was shown that neither PLA nor LAU competes for $\beta$-tubulin binding with PTX, however LAU is able to displace PLA from its target. ${ }^{46,64}$ Since these early competition experiments, various models have been employed to fully elucidate this unique protein region. ${ }^{41,70,72}$ Introduction of the E127A and K124A mutations did not change the proliferative phenotype of transfected cells treated with IXA. This suggests that the effects of replacing either glutamate 127 or lysine 124 with alanine are isolated to the region surrounding these amino acids. It was demonstrated in the previous chapter that GFP-tagged $\beta \mathrm{I}$-tubulin subunits are properly incorporated into functional microtubules, which is further supported by the lack of change in response to IXA between wild-type and either mutant.

Alterations to amino acid sequences as the result of acquired or engineered mutations can have substantial and varied impacts on protein function. For example, changing the internal interactions between peptide sequences can contribute to a protein's overall threedimensional structure or its ability to carry out a catalytic activity. Specifically relating to $\beta$ tubulin monomers, mutations at the carboxy-terminus can influence microtubule function through irregular associations with $\mathrm{MAPs},{ }^{88}$ while others may have global effects on microtubule polymerisation and stability. ${ }^{18,89}$ Clinically, these phenomena impact on the ability to treat multi-drug resistant tumours harbouring mutations that affect MTA activity, particularly following long-term exposure in chronic diseases.

The steric and electrostatic changes that can result from mutations in $\beta$-tubulin peptide sequences, independent of localisation in or near binding sites, can lead to global structural changes due to altered conformations. In addition to effects within individual monomers, this may also extend to aberrant inter-subunit and heterodimer associations. ${ }^{90}$ In this vein, the mutations under investigation here were designed to limit their impact on important proteinprotein interactions to isolate any drug binding effects, while also maximising the loss of compound-amino acid interaction to amplify those effects. This is particularly relevant as any interactions between PLA/LAU and either E127 or K124 would span the inter-protofilament space between adjacent $\beta$-subunits. Therefore, despite careful consideration, there still remains the possibility that any substitutions at these locations, particularly involving changes to electrostatic associations, may have a destabilising effect on microtubules independent of drug activity. The mutations were designed based on recently published x-ray crystallography 
studies of the PLA/LAU binding site, ${ }^{72}$ as well as in silico modelling carried out by Assoc. Prof. Paul Teesdale-Spittle (unpublished).

These models suggested that substituting both glutamate 127 and lysine 124 to an alanine would affect the postulated inter-protofilament binding of PLA and LAU to the adjacent $\beta$ tubulin subunit. Alanine is the smallest amino acid with an inert, non-polar methyl side chain replacing the long, polar carbonyl and amino side chains of glutamate and lysine, respectively. Based on the models, the K124A substitution would disrupt hydrogen bonding between the amino lysine side chain and the O6 and O7 atoms of PLA, and the O15 and O16 atoms of LAU. A further rationale used to predict the importance of these potential interactions was the $>1000$-fold loss of in vitro activity for PLA analogues for which the implicated oxygen-containing groups were altered. ${ }^{76}$ The E127A mutation was predicted to disrupt a potential interaction between the glutamate carbonyl side chain and a methyl group at the 11-position on LAU, or potential hydrogen bonds with the same oxygens as K124 if the alignment of the model with respect to the adjacent subunit was incorrect. If these interactions are real and important to PLA/LAU binding, introduction of an alanine in place of either of the amino acids should hinder that binding. However, recent studies have shown that both E127 and K124 participate in inter-protofilament interactions in the absence of PLA or LAU, forming salt bridges with K338 and E230/Q293, respectively. ${ }^{75,91}$ The reduced size and polarity of alanine in place of these amino acids would likely disrupt these interactions, with the potential for widespread changes to tubulin structure and function. While a study investigating the point mutation Q293M did not observe microtubule instability, it is possible that the substitution of a glycine to a methionine maintained the inter-protofilament saltbridge. However, the initial concentration-response results for IXA treated cells does not indicate any change in proliferation as a result of these mutations. This therefore allowed the results seen for PLA and LAU treatment to be directly related to drug-protein interactions.

MTT cell proliferation assays used in this study involved 4 biological repeats, in duplicate, of wild-type, D297I, E127A, K124A and mock transfected HEK293 cells treated with all four MSA compounds. This produced a large data set that gave consistent results with low levels of variation, enhancing the ability to determine small but significant differences in the concentration-response between mutant and wild-type cell populations. This was an important quality due to the uncertain extent and duration of exogenous $\beta I$-tubulin expression, as outlined in chapter 2. A shorter drug incubation period of $48 \mathrm{~h}$ was also trialled to address this. However, it failed to produce useful concentration-response curves (results 
not shown). Furthermore, the results gathered here were internally validated by the significant increase in resistance of D297I mutant $\beta$ I-tubulin transfected cells to PLA compared to wildtype. This increase in $\mathrm{IC}_{50}$ of $62 \pm 1 \%$ is similar to that reported in another study looking at mutations of amino acids related to PLA and LAU binding. ${ }^{74}$ This particular substitution of aspartate 297 to an isoleucine was shown to confer resistance to both compounds by disrupting hydrogen bonding interactions within the main PLA/LAU binding pocket. This result therefore gives weight to the effects seen for other mutations tested in the current study.

However, based on the previously published findings regarding the D297I mutation, resistance to LAU would have also be expected. In fact, LAU exhibited an approximate 10fold increase in $\mathrm{IC}_{50}$ across the different transfection types compared to values reported in related literature. ${ }^{74}$ This suggests some form of degradation of the compound, which was somewhat expected due to the low concentration and suspected age of the stock supply. Some evidence suggests that some of the compound in this supply may have converted to the far less potent isolaulimalide, which has an $\mathrm{IC}_{50}$ of $20 \mu \mathrm{M}$ in the MDA-MB-435 breast cancer cell line. ${ }^{92}$ Due to its epoxide moiety, LAU is a relatively unstable compound, rapidly rearranging to isolaulimalide on exposure to acid. If this is the reason for the high $\mathrm{IC}_{50}$ concentrations, the lack of this epoxide moiety would likely remove any interaction with $\mathrm{K} 124$, and perhaps E127, as hydrogen bonding was predicted between that particular oxygen and the amino acids. ${ }^{72}$ However, it is impossible to conclude the true identity of the LAU used in this experiment without utilising analytical chemistry techniques. As a result of the irregular performance of LAU in these assays the results were omitted from the conclusions drawn, and subsequent experiments focussed solely on PLA.

In cell cultures treated with PLA neither the E127A nor the K124A mutations produced a significant difference in $\mathrm{IC}_{50}$ when compared to wild-type control. Furthermore, no significant difference was observed between wild-type and mock transfected cells, supporting the aforementioned evidence that the introduction of GFP-tagged exogenous $\beta \mathrm{I}$-tubulin is not detrimental to the normal proliferation of cells. The lack of effect seen for the E127A mutation is not entirely unexpected due to the large modelled distance between any hydrogen bond donors and the carbonyl glutamate side chain. This also suggests that altering the interprotofilament salt bridge formation between E127 and K338 does not impact on PLA binding, whereas modelling carried out with LAU suggests that this interaction does contribute to the thermodynamics of drug-tubulin binding. ${ }^{75}$ The lack of change in concentration-response for the K124A mutant cells is more surprising. While this does 
suggest that the predicted hydrogen bonding between the lysine side chain and oxygen atoms on PLA is not essential, it does not rule out compensatory effects from neighbouring, namely D120 and E123. ${ }^{72}$ It should also be noted that the loss of transgene over the course of the longer MTT assay may have removed any changes that would have been observed during the shorter flow cytometry and microscopy experiments. This would also support the dissonance between results seen for PLA in the MTT assay versus those described below, for which analysis was carried out much sooner following tranfection. Furthermore, it is not clear what contributions these interactions make to the overall binding and subsequent microtubule stabilisation by PLA. Inter-protofilament modelling of LAU binding suggests that major hydrogen bonds are formed with amino acids in the main protein pocket, as well as E53 and G56 on the adjacent subunit, with only minor energetic contributions made by other amino acids. $^{75,91}$ In terms of the role of these potential interactions, PLA and LAU binding to the main pocket on $\beta$-tubulin promotes re-organisation of the M-loop tubulin substructure. The M-loop is involved in the association of adjacent protofilaments during microtubule polymerisation, and these PLA/LAU-induced conformational changes reduce the energy requirements for this to occur. It is therefore reasonable to suggest that contributions made to this inter-protofilament interaction by direct drug binding could further promote microtubule polymerisation and stability.

\subsubsection{The changes to cell cycle arrest caused by peloruside A with E127A mutant BI-tubulin}

A characteristic of MSA cytotoxicity is that, prior to apoptotic cell death, the reduced dynamicity of microtubules leads to the inhibition of mitosis and a build-up of cells in the $\mathrm{G}_{2} / \mathrm{M}$ phase of the cell cycle. This effect can be analysed by flow cytometry, staining genomic DNA with PI to determine copy number as an indicator of cell cycle progression. An issue with this technique is that the standard protocol uses ethanol to fix cells, which at the same time permeabilises them to the impermeable PI. However, it is well documented that this has a deleterious effect GFP signal, ${ }^{93,94}$ which is detrimental to the power of this analysis that lies in the ability to analyse GFP positive (transfected) cells in isolation. Therefore, an alternative protocol was sought. Fixation by crosslinking with paraformaldehyde is also commonly used, however it is associated with poor PI signal output due to fragmentation of DNA, ${ }^{95}$ signified by large coefficients of variance in the resulting histograms. Therefore, a zinc-based fixative was used as an alternative to ethanol as it has 
been shown to preserve DNA integrity well over long periods of time, while retaining GFP signal. ${ }^{96}$ Due to the evidently compromised supply of LAU and the fact that K124A has been studied in the context of cell cycle arrest by another member of the Miller lab group (results unpublished), this and all remaining experiments focussed on the E127A point mutation and PLA.

The resulting experiments showed a typical $\mathrm{G}_{2} / \mathrm{M}$ block in GFP positive wild-type cells treated with both IXA and PLA. Based on the the results from the cell proliferation assays, this outcome was expected. As GFP-tagged $\beta I$-tubulin-containing microtubules have been proven functional, no change to the effects on these cells should occur. The fidelity of wildtype exogenous $\beta$ I-tubulin was further supported by the similar results seen for both GFP positive and negative sub-populations. The quality of the PI histograms was noticeably poorer for wild-type samples compared to E127A mutants, however it is unclear as to the reason for this. Regardless, they still produced defensible values for cell cycle percentages, comparable to the mutation samples.

Interestingly, the proportion of cells in the $G_{2} / M$ phase was significantly lower for E127A transfected cells treated with 40 and $100 \mathrm{nM}$ of both IXA and PLA compared to wild-type. However, there were no such differences seen for the GFP negative sub-populations. This is in contrast to the result observed in the proliferation assays described above. The similarity in effect between both the taxane compound IXA and PLA suggests that introduction of the mutation has a global effect on microtubule stability, causing MSA resistance regardless of binding site. As mentioned previously, this effect was somewhat expected to occur for these mutations due to predicted salt bridge associations between E127 and K124 and amino acids on the adjacent subunit. There is also precedent for similar outcomes from single amino acid substitutions in the PLA/LAU binding site. A study investigating the $\beta$-tubulin point mutations V225W and N339L showed that both produced unstable microtubules as identified by the resistance of transfected cells to both PTX and PLA. ${ }^{74}$ The former substituted V225 with an amino acid affording greater steric hindrance (tryptophan) aimed at reducing the strength of PLA and LAU binding. The purpose of the latter was to switch hydrogen bonding from PLA to LAU. However, instead, both mutations had the effect of MSA-independent tubulin destabilisation. They also exhibited reduced microtubule networks in the absence of drug, as viewed with immunocytochemistry. This is a previously defined phenotype for global destabilisation, also seen for other PTX-resistant mutants. ${ }^{89}$ This may also explain the 
poor definition of GFP fluorescent microtubule structures and increased aggregates compared to all other transfected cells, as observed by confocal microscopy in chapter 2 .

\subsubsection{In situ polymerisation of exogenous wild-type BI-tubulin treated with peloruside $A$}

To determine if introduction of the E127A mutation has an impact on tubulin polymerisation caused by PLA, the in situ analysis of tubulin solubility was pursued. This is a commonly used technique to measure the effect of MSAs, with normal results showing a concentrationdependent increase in the proportion of polymerised (insoluble) tubulin due to enhance microtubule stabilisation. ${ }^{73,74}$ However, as observed for the transfection efficiency western blotting procedure described in chapter 2, this experiment was unsuccessful. Again, it is unclear why no defensible $\beta$-tubulin bands were observed in all but one of the blots, besides the possible explanations already outlined. An additional source of disruption specific to this methodology is the hypotonic lysis buffer used for lysate preparation. A very different treatment of cells is required in this protocol compared to that used for transfection efficiency experiment, so as to retain tubulin protein in the soluble and insoluble fractions. To this end, the non-ionic lysis buffer Nonidet ${ }^{\mathrm{TM}} \mathrm{P}-40$ has been used successfully in past experiments with HEk293 cells. $^{74}$ Unfortunately, this buffer is no longer manufactured, and in response a number of substitutes have been recently trialled. ${ }^{97}$ The selection of $0.15 \%$ Tergitol $^{\circledR}$ as the detergent and concentration used in this project was based on the results from those trials. However, as they were carried out with 1A9 ovarian cancer cells, physiological differences between the two may lead to differences in the lysis conditions required. One blot did show faint bands at the correct molecular weight for GFP-tagged $\beta$-tubulin (75 kDa) for PLAtreated wild-type cells. This showed a small increase in polymerised tubulin with increasing PLA concentration. While this provides very little meaningful information on its own, it does support the other evidence gathered that shows GFP-tagged wild-type $\beta I$-tubulin to maintain functionality. However, it is impossible to draw conclusions from this single result.

\subsubsection{Qualitative assessment of GFP-tagged microtubule functionality in response to} peloruside $A$

As well as the quantitative assessment of MSA activity in E127A mutant $\beta I$-tubulin transfected cells, it is also valuable to observe visual changes to their microtubule function in 
response to the compounds. Confocal microscopy of immunocytochemistry labelled cell samples was employed for this purpose. Consistent with the results produced by the other experiments, wild-type transfected cells displayed GFP-tagged $\beta$ I-tubulin-containing microtubules exhibiting normal function in the absence of any drug. Furthermore, when treated with both IXA and PLA $(100 \mathrm{nM})$, the expected response was observed, being the formation of multiple asters as an indicator of mitotic block. These are areas of condensed tubulin observed around condensed chromatin within dividing cells and an increase in their intensity and frequency suggests stabilisation of microtubules prior to apoptosis. Similar effects have been seen for PTX and PLA-treated cells in previous studies. ${ }^{58,98}$ The use of a fluorophore to label $\beta$-tubulin with an emission spectra that does overlap substantially with GFP allowed the parallel visualisation of both GFP-tagged and total $\beta$-tubulin in samples. This showed that many of the dividing cells imaged were successfully transfected, and more importantly, showed GFP-tagged microtubules facilitating mitotic processes, such as through spindle formation.

In contrast, cells transfected with GFP-tagged E127A $\beta$ I-tubulin showed signs of abnormal microtubule function. Untreated control cells, showed few dividing cells, however none were seen to involve GFP-fluorescent mitotic apparatus. Furthermore, while aster formation with drug treatment in non-transfected cells was similar to that seen for wild-type transfected samples, E127A mutant cells showed little evidence of mitotic arrest in response to either IXA or PLA. In addition, the mutant tubulin-expressing cells showed signs of aberrant microtubules. The GFP signal was less well defined and more diffuse compared to wild-type samples, while transfected cells showed more intracellular regions of intense fluorescence indicating GFP aggregation. This is similar to the effects seen for other point mutations reported in the literature that confer resistance to PTX by drug-independent microtubule destabilisation. ${ }^{89}$ Cells showed thinning microtubule networks with irregular nuclear morphologies. These observations correlate well to the results of the cell cycle analysis, with differences seen between the mutant and wild-type cells in terms of mitotic arrest, however with no differences between taxane and non-taxane MSA activity. 


\section{Chapter 4. Overall discussion}

\subsection{Final Discussion}

Since their discovery, MTAs have become extremely useful and widely-prescribed frontline chemotherapeutics for the treatment of a number of different cancer types. However, there is a clinical need to improve these therapies in order to combat multi-drug resistance and utilityreducing off-target toxicities. PLA and LAU represent promising candidates for this purpose, due to their potential advantages in treating resistant tumours. However, further development is required to enhance their pharmaceutical properties and ease of synthesis. Such development is greatly assisted by the complete understanding of a lead compound's target interactions.

The use of $\beta$-tubulin point mutations to investigate the biological relevance of specific amino acids to the binding mechanisms of MTAs has been reported extensively. Similar point mutations also occur in the development of multi-drug resistance among tumours overexposed to commonly administered taxane MSAs. ${ }^{83}$ Through these studies, the relative contributions of molecular interactions to overall target binding can be determined, and in doing so establish a hierarchy of peptide sequence importance to inform the rational design of more efficacious and targeted candidate drugs.

This study has produced evidence to help describe the role of two amino acids potentially involved in the inter-protofilament interaction between PLA and LAU and $\beta$-tubulin, a mode of binding not yet demonstrated beyond computational modelling. The results reported here suggest that neither E127 nor K124 play an essential role in PLA-induced microtubule stabilisation. This does not rule out their contribution to compound binding, as compensatory mechanisms may be involved or the differential power of this protocol may not be sufficient to reveal any significant effect. However, it is apparent that glutamate 127 is important to overall microtubule function and stability as its substitution to alanine resulted in a drugindependent tubulin destabilisation. While this effect was not observed in the cell proliferation assay, this may be due to the size of the effect requiring more sensitive techniques, such as the ability of flow cytometric cell cycle analysis to investigate transfected cells selectively. Furthermore, both the cell cycle and immunocytochemistry experiments that did reveal this destabilising effect involved much shorter time periods than did the MTT assays. Therefore, if mutant transfected cells have compromised mitotic abilities, it is likely that their presence in cell cultures will become diluted over the course of an assay. 


\subsection{Future Directions}

This investigation used transient transfections to introduce mutant $\beta I$-tubulin into cells to study the effects specific point mutations. As these single amino acid substitutions can have relatively small effects it would be useful for similar studies in the future to enhance the ability to detect such changes. For example, the clonal development of resistant cell lines in the presence of drug or complete replacement of endogenous protein by alternative gene editing techniques to increase the abundance of mutant $\beta$ I-tubulin would be useful. With improved differential power and resolution of effects, it may be easier to define specific amino acids based on their importance to drug interactions, a valuable tool for bioactivityguided analogue design.

One particular method that could be employed to enhance mutant gene expression in a cell model is the CRISPR-Cas9 system that utilises a bacteria-derived gene editing protein complex to incorporate a gene of interest into the genome of a cell. ${ }^{99}$ It uses specifically designed guide RNA to insert site-specific gene modifications through the annealing of double-stranded breaks. This system may also be used to engineer haploid cells. These have only one copy of each gene, which addresses the issue of uncertainty as to the proportions of mutant genes within cells that may be compensated for by endogenous sequences. While such cells would have to be validated to ensure normal microtubule function and cell proliferation phenotypes, they would provide a powerful cell model for which effects could be directly and confidently related to introduced mutations.

In terms of progressing the results of the current experiment, it would be useful to generate meaningful data from both western blotting experiments. To draw valid conclusion from the results, the true extent of mutant $\beta$ I-tubulin expression should be determined. Furthermore, it is desirable to confirm of the results from the cell cycle analysis and confocal microscopy experiments by assessing the in situ polymerisation behaviour of tubulin in E127 mutantexpressing cells. Furthermore, it would be very interesting to use fresh LAU in the same set of experiments, as both mutations are predicted by modelling to have greater effects on its mechanism of action compared to PLA. ${ }^{72}$ Another addition to help further characterise this inter-protofilament association would be the introduction of exogenous $\beta I$-tubulin with mutations at either of the amino acids E53 or G56, based on their high predicted contribution to LAU binding energies. ${ }^{75}$ 
The purpose of defining the PLA/LAU $\beta$-tubulin binding site is to inform the ongoing development of useful targeted therapeutic agents through structure-activity studies. While no clear direction is immediately revealed from these results, further elucidation of MSA interaction with adjacent heterodimers would be of great value. However, the destabilising effects of the E127A mutation, likely due to inhibition of salt bridge formation, may also prove useful. A potential strategy may be to strengthen these associations through drugprotein interactions targeted at the implicated amino acids on either side of the interprotofilament divide.

\subsection{Conclusion}

In conclusion, no evidence was found in this study that demonstrates a major role for the human $\beta$ I-tubulin amino acids glutamate 127 or lysine 124 in the mechanism of action of PLA or LAU. However, the results do suggest that E127 is important for the MTAindependent stability of microtubules, likely by inter-protofilament salt bridge formation. However, this needs to be further investigated through the repetition of unsuccessful experiments trialled here. Overall, this information supports the elucidation of the PLA/LAU $\beta$-tubulin region, hopefully contributing to future drug development efforts. 


\section{References}

1 Harvey, A. L., Clark, R. L., Mackay, S. P. \& Johnston, B. F. Current strategies for drug discovery through natural products. Expert Opinion On Drug Discovery 5, 559$568(2010)$.

2 Newman, D. J. \& Cragg, G. M. Natural products as sources of new drugs from 1981 to 2014. Journal of Natural Products 79, 629-661 (2016).

3 Faulkner, D. J. Marine natural products. Natural Product Reports 18, 1R-49R (2001).

4 Belarbi, E. H., Gomez, A. C., Chisti, Y., Garcifdeim EM.尹d aigd ss from marine sponges. Biotechnology Advances 21, 585-598 (2003).

5 Miller, J. H., Singh, A. J. \& Northcote, P. T. Microtubule-stabilizing drugs from marine sponges: focus on peloruside A and zampanolide. Marine Drugs 8, 1059-1079 (2010).

6 Seddon, G. et al. Drug design for ever, from hype to hope. Journal Of ComputerAided Molecular Design 26, 137-150 (2012).

7 Siegel, R., Naishadham, D. \& Jemal, A. Cancer statistics, 2012. CA: A Cancer Journal For Clinicians 62, 10-29 (2012).

8 MOH. Cancer: New registrations and deaths 2012. (2015).

9 Jemal, A., Center, M. M., DeSantis, C. \& Ward, E. M. Global patterns of cancer incidence and mortality rates and trends. Cancer Epidemiology Biomarkers \& Prevention 19, 1893-1907 (2010).

10 Hanahan, D. \& Weinberg, R. A. Hallmarks of cancer: the next generation. Cell 144, 646-674 (2011). 
11 Costello, L. C. \& Franklin, R. B. The genetic/metabolic transformation concept of carcinogenesis. Cancer and Metastasis Reviews 31, 123-130 (2012).

12 Vineis, P., Schatzkin, A. \& Potter, J. D. Models of carcinogenesis: an overview. Carcinogenesis 31, 1703-1709 (2010).

13 Scheel, C. \& Weinberg, R. A. in Seminars in cancer biology. 396-403 (Elsevier).

14 Bindal, R. K., Sawaya, R., Leavens, M. E. \& Lee, J. J. Surgical treatment of multiple brain metastases. Journal Of Neurosurgery 79, 210-216 (1993).

15 DeAngelis, L. M. Brain tumors. New England Journal of Medicine 344, 114-123 (2001).

16 Evans, W. E. \& McLeod, H. L. Pharmacogenomics - drug disposition, drug targets, and side effects. New England Journal of Medicine 348, 538-549 (2003).

17 Borst, P., Evers, R., Kool, M. \& Wijnholds, J. A family of drug transporters: the multidrug resistance-associated proteins. Journal of the National Cancer Institute $\mathbf{9 2 ,}$ 1295-1302 (2000).

18 Kavallaris, M. Microtubules and resistance to tubulin-binding agents. Nature Reviews Cancer 10, 194-204 (2010).

19 Nogales, E. Structural insights into microtubule function. Annual Review Of Biophysics And Biomolecular Structure 30, 397-420 (2001).

20 Nogales, E., Whittaker, M., Milligan, R. A. \& Downing, K. H. High-resolution model of the microtubule. Cell 96, 79-88 (1999).

21 Chrétien, D., Metoz, F., Verde, F., Karsenti, E. \& Wade, R. Lattice defects in microtubules: protofilament numbers vary within individual microtubules. The Journal Of Cell Biology 117, 1031-1040 (1992). 
22 Desai, A. \& Mitchison, T. J. Microtubule polymerization dynamics. Annual Review Of Cell And Developmental Biology 13, 83-117 (1997).

23 Luduena, R. Are tubulin isotypes functionally significant. Molecular Biology of the Cell 4, 445 (1993).

24 Nogales, E., Wang, H.-W. \& Niederstrasser, H. Tubulin rings: which way do they curve? Current Opinion In Structural Biology 13, 256-261 (2003).

25 Jánosi, I. M., Chrétien, D. \& Flyvbjerg, H. Structural microtubule cap: Stability, catastrophe, rescue, andthird state. Biophysical Journal 83, 1317-1330 (2002).

26 Rusan, N. M., Fagerstrom, C. J., Yvon, A.-M. C. \& Wadsworth, P. Cell cycledependent changes in microtubule dynamics in living cells expressing green fluorescent protein- $\alpha$ tubulin. Molecular Biology Of The Cell 12, 971-980 (2001).

27 Slautterback, D. B. Cytoplasmic microtubules. The Journal Of Cell Biology 18, 367388 (1963).

28 Borisy, G. G. \& Taylor, E. The mechanism of action of colchicine. The Journal Of Cell Biology 34, 525-533 (1967).

29 Kelling, J., Sullivan, K., Wilson, L. \& Jordan, M. A. Suppression of centromere dynamics by Taxol ${ }^{\circledR}$ in living osteosarcoma cells. Cancer Research 63, 2794-2801 (2003).

30 Dumontet, C. \& Jordan, M. A. Microtubule-binding agents: a dynamic field of cancer therapeutics. Nature Reviews Drug Discovery 9, 790-803 (2010).

31 Jordan, M. A. \& Wilson, L. Microtubules as a target for anticancer drugs. Nature Reviews Cancer 4, 253-265 (2004). 
32 Komlodi-Pasztor, E., Sackett, D. L. \& Fojo, A. T. Inhibitors targeting mitosis: tales of how great drugs against a promising target were brought down by a flawed rationale. Clinical Cancer Research 18, 51-63 (2012).

33 Field, J. J., Kanakkanthara, A. \& Miller, J. H. Microtubule-targeting agents are clinically successful due to both mitotic and interphase impairment of microtubule function. Bioorganic \& Medicinal Chemistry 22, 5050-5059 (2014).

34 Gottesman, M. M. Mechanisms of cancer drug resistance. Annual Review Of Medicine 53, 615-627 (2002).

35 Guillemard, V. \& Saragovi, H. U. Taxane-antibody conjugates afford potent cytotoxicity, enhanced solubility, and tumor target selectivity. Cancer Research 61, 694-699 (2001).

36 Gigant, B., Cormier, A., Dorléans, A., Ravelli, R. \& Knossow, M. in Tubulin-Binding Agents 259-278 (Springer, 2008).

37 Ravelli, R. B. et al. Insight into tubulin regulation from a complex with colchicine and a stathmin-like domain. Nature 428, 198-202 (2004).

38 Kumar, A. et al. A novel microtubule depolymerizing colchicine analogue triggers apoptosis and autophagy in HCT-116 colon cancer cells. Cell Biochemistry And Function 34, 69-81 (2016).

39 Yue, Q.-X., Liu, X. \& Guo, D.-A. Microtubule-binding natural products for cancer therapy. Planta Medica 76, 1037-1043 (2010).

40 Yang, H., Ganguly, A. \& Cabral, F. Inhibition of cell migration and cell division correlates with distinct effects of microtubule inhibiting drugs. Journal of Biological Chemistry 285, 32242-32250 (2010).

41 Huzil, J. T. et al. A unique mode of microtubule stabilization induced by peloruside A. Journal Of Molecular Biology 378, 1016-1030 (2008). 
42 Wani, M. C., Taylor, H. L., Wall, M. E., Coggon, P. \& McPhail, A. T. Plant antitumor agents. VI. Isolation and structure of taxol, a novel antileukemic and antitumor agent from Taxus brevifolia. Journal of the American Chemical Society 93, 2325-2327 (1971).

43 Schiff, P. B., Fant, J. \& Horwitz, S. B. Promotion of microtubule assembly in vitro by taxol. Nature (1979).

44 Elie-Caille, C. et al. Straight GDP-tubulin protofilaments form in the presence of taxol. Current Biology 17, 1765-1770 (2007).

45 Gelderblom, H., Verweij, J., Nooter, K. \& Sparreboom, A. Cremophor EL: the drawbacks and advantages of vehicle selection for drug formulation. European Journal of Cancer 37, 1590-1598 (2001).

46 Gaitanos, T. N. et al. Peloruside A does not bind to the taxoid site on $\beta$-tubulin and retains its activity in multidrug-resistant cell lines. Cancer Research 64, 5063-5067 (2004).

47 Rohena, C. C. \& Mooberry, S. L. Recent progress with microtubule stabilizers: new compounds, binding modes and cellular activities. Natural Product Reports 31, 335355 (2014).

48 Bollag, D. M. et al. Epothilones, a new class of microtubule-stabilizing agents with a taxol-like mechanism of action. Cancer research 55, 2325-2333 (1995).

49 Shen, H., Lee, F. \& Gan, J. Ixabepilone, a novel microtubule-targeting agent for breast cancer, is a substrate for P-glycoprotein (P-gp/MDR1/ABCB1) but not breast cancer resistance protein (BCRP/ABCG2). Journal of Pharmacology and Experimental Therapeutics 337, 423-432 (2011). 
50 Wozniak, K. M. et al. Comparison of neuropathy-inducing effects of eribulin mesylate, paclitaxel, and ixabepilone in mice. Cancer Research 71, 3952-3962 (2011).

51 Conlin, A., Fornier, M., Hudis, C., Kar, S. \& Kirkpatrick, P. Ixabepilone. Nature Review Drug Discovery 6, 953-954 (2007).

$52 \mathrm{Li}$, J. et al. Potent taccalonolides, AF and AJ, inform significant structure-activity relationships and tubulin as the binding site of these microtubule stabilizers. Journal of the American Chemical Society 133, 19064-19067 (2011).

53 Li, J., Risinger, A. L. \& Mooberry, S. L. Taccalonolide microtubule stabilizers. Bioorganic \& Medicinal Chemistry 22, 5091-5096 (2014).

54 Field, J. J. et al. Microtubule-stabilizing activity of zampanolide, a potent macrolide isolated from the Tongan marine sponge Cacospongia mycofijiensis. Journal of Medicinal Chemistry 52, 7328-7332 (2009).

55 Larsen, E. M., Wilson, M. R., Zajicek, J. \& Taylor, R. E. Conformational Preferences of Zampanolide and Dactylolide. Organic Letters 15, 5246-5249 (2013).

56 Buey, R. M. et al. Cyclostreptin binds covalently to microtubule pores and lumenal taxoid binding sites. Nature Chemical Biology 3, 117-125 (2007).

57 West, L. M., Northcote, P. T. \& Battershill, C. N. Peloruside A: a potent cytotoxic macrolide isolated from the new zealand marine sponge Mycale sp. The Journal of Organic Chemistry 65, 445-449 (2000).

58 Hood, K. A. et al. Peloruside A, a novel antimitotic agent with paclitaxel-like microtubule-stabilizing activity. Cancer Research 62, 3356-3360 (2002).

59 Meyer, C. J. et al. Peloruside A inhibits growth of human lung and breast tumor xenografts in an athymic nu/nu mouse model. Molecular Cancer Therapeutics 14, 1816-1823 (2015). 
60 Wilmes, A., Bargh, K., Kelly, C., Northcote, P. T. \& Miller, J. H. Peloruside A synergizes with other microtubule stabilizing agents in cultured cancer cell lines. Molecular Pharmaceutics 4, 269-280 (2007).

61 Corley, D. G., Herb, R., Moore, R. E., Scheuer, P. J. \& Paul, V. J. Laulimalides. New potent cytotoxic macrolides from a marine sponge and a nudibranch predator. The Journal of Organic Chemistry 53, 3644-3646 (1988).

62 Quinoa, E., Kakou, Y. \& Crews, P. Fijianolides, polyketide heterocycles from a marine sponge. The Journal of Organic Chemistry 53, 3642-3644 (1988).

63 Mooberry, S. L., Tien, G., Hernandez, A. H., Plubrukarn, A. \& Davidson, B. S. Laulimalide and isolaulimalide, new paclitaxel-like microtubule-stabilizing agents. Cancer Research 59, 653-660 (1999).

64 Pryor, D. E. et al. The microtubule stabilizing agent laulimalide does not bind in the taxoid site, kills cells resistant to paclitaxel and epothilones, and may not require its epoxide moiety for activity. Biochemistry 41, 9109-9115 (2002).

65 Johnson, T. A. et al. Sponge-derived fijianolide polyketide class: further evaluation of their structural and cytotoxicity properties. Journal Of Medicinal Chemistry 50, 37953803 (2007).

66 Liu, J. et al. In vitro and in vivo anticancer activities of synthetic (-)-laulimalide, a marine natural product microtubule stabilizing agent. Anticancer Research 27, 15091518 (2007).

67 Pineda, O. et al. Computational comparison of microtubule-stabilising agents laulimalide and peloruside with taxol and colchicine. Bioorganic \& Medicinal Chemistry Letters 14, 4825-4829, doi:http://dx.doi.org/10.1016/j.bmcl.2004.07.053 (2004). 
68 Löwe, J., Li, H., Downing, K. \& Nogales, E. Refined structure of $\alpha \beta$-tubulin at $3.5 \AA$ resolution. Journal Of Molecular Biology 313, 1045-1057 (2001).

69 Thepchatri, P. et al. Conformations of Laulimalide in DMSO-d6. Journal of the American Chemical Society 127, 12838-12846 (2005).

70 Nguyen, T. L., Xu, X., Gussio, R., Ghosh, A. K. \& Hamel, E. The assembly-inducing laulimalide/peloruside a binding site on tubulin: molecular modeling and biochemical studies with $[3 \mathrm{H}]$ peloruside A. Journal of Chemical Information and Modeling 50, 2019-2028 (2010).

71 Khrapunovich-Baine, M. et al. Hallmarks of molecular action of microtubule stabilizing agents effects of epothilone $b$, ixabepilone, peloruside a, and laulimalide on microtubule conformation. Journal of Biological Chemistry 286, 11765-11778 (2011).

72 Prota, A. E. et al. Structural basis of microtubule stabilization by laulimalide and peloruside A. Angewandte Chemie International Edition 53, 1621-1625 (2014).

73 Kanakkanthara, A., Eras, J., T Northcote, P., Cabral, F. \& H Miller, J. Resistance to peloruside a and laulimalide: functional significance of acquired $\beta i$-tubulin mutations at sites important for drug-tubulin binding. Current Cancer Drug Targets 14, 79-90 (2014).

74 Kanakkanthara, A. et al. $\beta$ I-tubulin mutations in the laulimalide/peloruside binding site mediate drug sensitivity by altering drug-tubulin interactions and microtubule stability. Cancer Letters 365, 251-260 (2015).

75 Churchill, C. D., Klobukowski, M. \& Tuszynski, J. A. The unique binding mode of laulimalide to two tubulin protofilaments. Chemical Biology \& Drug Design 86, 190199 (2015). 
76 Wullschleger, C. W., Gertsch, J. \& Altmann, K. H. Synthesis and biological activity of 7, 8, 9-trideoxy-and 7R desTHP-peloruside A. Chemistry-A European Journal 19, 13105-13111 (2013).

77 Chesnoy, S. \& Huang, L. Structure and function of lipid-DNA complexes for gene delivery. Annual Review Of Biophysics And Biomolecular Structure 29, 27-47 (2000).

78 Bishop, C. L. et al. Role for centromeric heterochromatin and PML nuclear bodies in the cellular response to foreign DNA. Molecular And Cellular Biology 26, 2583-2594 (2006).

79 Riu, E., Chen, Z.-Y., Xu, H., He, C.-Y. \& Kay, M. A. Histone modifications are associated with the persistence or silencing of vector-mediated transgene expression in vivo. Molecular Therapy 15, 1348-1355 (2007).

80 Brooks, A. R. et al. Transcriptional silencing is associated with extensive methylation of the CMV promoter following adenoviral gene delivery to muscle. The Journal of Gene Medicine 6, 395-404 (2004).

81 Qiu, G.-H. et al. Recognition and suppression of transfected plasmids by protein ZNF511-PRAP1, a potential molecular barrier to transgene expression. Molecular Therapy 19, 1478-1486 (2011).

82 Wang, Y. et al. Mutations at Leucine 215 of $\beta$-tubulin affect paclitaxel sensitivity by two distinct mechanisms. Biochemistry 45, 185-194 (2006).

83 Kanakkanthara, A. et al. Peloruside-and laulimalide-resistant human ovarian carcinoma cells have $\beta$ I-tubulin mutations and altered expression of $\beta$ II-and $\beta$ IIItubulin isotypes. Molecular Cancer Therapeutics 10, 1419-1429 (2011).

84 Mosmann, T. Rapid colorimetric assay for cellular growth and survival: application to proliferation and cytotoxicity assays. Journal Of Immunological Methods 65, 55-63 (1983). 
85 Berridge, M. V., Herst, P. M. \& Tan, A. S. Tetrazolium dyes as tools in cell biology: new insights into their cellular reduction. Biotechnology Annual Review 11, 127-152 (2005).

86 Giannakakou, P. et al. Paclitaxel-resistant human ovarian cancer cells have mutant $\beta$ tubulins that exhibit impaired paclitaxel-driven polymerization. Journal of Biological Chemistry 272, 17118-17125 (1997).

87 Dumontet, C., Jordan, M. A. \& Lee, F. F. Ixabepilone: targeting BIII-tubulin expression in taxane-resistant malignancies. Molecular Cancer Therapeutics 8, 17-25 (2009).

88 Verdier-Pinard, P. et al. Tubulin proteomics: towards breaking the code. Analytical Biochemistry 384, 197 (2009).

89 Yin, S., Bhattacharya, R. \& Cabral, F. Human mutations that confer paclitaxel resistance. Molecular Cancer Therapeutics 9, 327-335 (2010).

90 Sheinerman, F. B., Norel, R. \& Honig, B. Electrostatic aspects of protein-protein interactions. Current Opinion In Structural Biology 10, 153-159 (2000).

91 Churchill, C. D., Klobukowski, M. \& Tuszynski, J. A. Analysis of the binding mode of laulimalide to microtubules: Establishing a laulimalide-tubulin pharmacophore. Journal of Biomolecular Structure and Dynamics 34, 1455-1469 (2016).

92 Gollner, A., Altmann, K. H., Gertsch, J. \& Mulzer, J. The laulimalide family: total synthesis and biological evaluation of neolaulimalide, isolaulimalide, laulimalide and a nonnatural analogue. Chemistry-A European Journal 15, 5979-5997 (2009).

93 Kalejta, R. F., Brideau, A. D., Banfield, B. W. \& Beavis, A. J. An integral membrane green fluorescent protein marker, Us9-GFP, is quantitatively retained in cells during propidium iodide-based cell cycle analysis by flow cytometry. Experimental Cell Research 248, 322-328 (1999). 
94 Houseknecht, J. B., Temple, J. S. \& Bateman Jr, R. C. Analysis of cell-cycle profiles in transfected cells using a membrane-targeted GFP. Biotechniques 24, 348-354 (1998).

95 Gillio-Tos, A. et al. Efficient DNA extraction from 25-year-old paraffin-embedded tissues: study of 365 samples. Pathology 39, 345-348 (2007).

96 Jensen, U. B., Owens, D. M., Pedersen, S. \& Christensen, R. Zinc fixation preserves flow cytometry scatter and fluorescence parameters and allows simultaneous analysis of DNA content and synthesis, and intracellular and surface epitopes. Cytometry Part A 77, 798-804 (2010).

97 Sinha, S., Field, J. J. \& Miller, J. H. Use of substitute Nonidet P-40 nonionic detergents in intracellular tubulin polymerization assays for screening of microtubule targeting agents. Biochemistry and Cell Biology (2017).

98 Torres, K. \& Horwitz, S. B. Mechanisms of Taxol-induced cell death are concentration dependent. Cancer Research 58, 3620-3626 (1998).

99 Doudna, J. A. \& Charpentier, E. The new frontier of genome engineering with CRISPR-Cas9. Science 346, 1258096 (2014). 